\title{
Streamlining the use of BOLD specimen data to record species distributions: a case study with ten Nearctic species of Microgastrinae (Hymenoptera: Braconidae)
}

\author{
Jose L Fernandez-Triana ${ }^{\dagger}$, Lyubomir Penev ${ }^{\ddagger}$, Sujeevan Ratnasingham ${ }^{\S}$, M. Alex Smithl, \\ Jayme Sones ${ }^{\S}$, Angela Telfer ${ }^{\S}$, Jeremy R. deWaard", Paul D. N. Hebert" \\ † Canadian National Collection of Insects, Ottawa, and the Biodiversity Institute of Ontario, University of Guelph, Ottawa, \\ Canada \\ $\ddagger$ Pensoft, Sofia, Bulgaria \\ $\S$ University of Guelph, Guelph, Canada \\ | Department of Integrative Biology, Guelph, Canada \\ I Biodiversity Institute of Ontario, University of Guelph, Guelph, Canada
}

\begin{abstract}
Corresponding author: Jose L Fernandez-Triana (iftriana@uoguelph.ca)
Academic editor: Dominique Zimmermann

Received: 11 Oct 2014 | Accepted: 24 Oct 2014 | Published: 29 Oct 2014

Citation: Fernandez-Triana J, Penev L, Ratnasingham S, Smith M, Sones J, Telfer A, deWaard J, Hebert P (2014) Streamlining the use of BOLD specimen data to record species distributions: a case study with ten Nearctic species of Microgastrinae (Hymenoptera: Braconidae). Biodiversity Data Journal 2: e4153. doi: 10.3897/BDJ.2.e4153
\end{abstract}

\begin{abstract}
The Barcode of Life Data Systems (BOLD) is designed to support the generation and application of DNA barcode data, but it also provides a unique source of data with potential for many research uses. This paper explores the streamlining of BOLD specimen data to record species distributions - and its fast publication using the Biodiversity Data Journal (BDJ), and its authoring platform, the Pensoft Writing Tool (PWT). We selected a sample of 630 specimens and 10 species of a highly diverse group of parasitoid wasps (Hymenoptera: Braconidae, Microgastrinae) from the Nearctic region and used the information in BOLD to uncover a significant number of new records (of locality, provinces, territories and states). By converting specimen information (such as locality, collection date, collector, voucher depository) from the BOLD platform to the Excel template provided by the PWT, it is possible to quickly upload and generate long lists of "Material Examined"
\end{abstract}


for papers discussing taxonomy, ecology and/or new distribution records of species. For the vast majority of publications including DNA barcodes, the generation and publication of ancillary data associated with the barcoded material is seldom highlighted and often disregarded, and the analysis of those data sets to uncover new distribution patterns of species has rarely been explored, even though many BOLD records represent new and/or significant discoveries. The introduction of journals specializing in - and streamlining - the release of these datasets, such as the BDJ, should facilitate thorough analysis of these records, as shown in this paper.

\section{Keywords}

Species distribution records, streamlining data, Barcode of Life Data Systems, Pensoft Writing Tool, Microgastrinae, Nearctic

\section{Introduction}

The Barcode of Life Data Systems (BOLD) is designed to support the generation and application of DNA barcode data. The platform consists of four main modules: a data portal, a database of barcode clusters, an educational portal, and a data collection workbench. Currently almost 4 million sequences (over 3.4 million of them DNA barcodes) are stored in BOLD, including coverage for more than $143 \mathrm{~K}$ animal species, $53 \mathrm{~K}$ plant species, and $16 \mathrm{~K}$ fungi and other species (http://www.boldsystems.org/, accessed on October 09, 2014). This immense platform provides a unique source of data with potential for many research uses. For example, the use of sequences in BOLD has been greatly expanded in the past few years, and dozens of publications using BOLD exist.

A less known and explored avenue is the use of specimen data (also stored in BOLD), which has a great potential to reveal new biodiversity information as well. This paper explores the streamlining of BOLD specimen data to record species distributions. For that we use the Biodiversity Data Journal (BDJ), and its authoring platform, the Pensoft Writing Tool (PWT), which is a community peer-reviewed, open-access, comprehensive online platform, designed to accelerate publishing, dissemination and sharing of biodiversityrelated data of any kind (http://biodiversitydatajournal.com/about\#FocusandScope).

A highly diverse group of insects (parasitoid wasps of the family Braconidae, subfamily Microgastrinae) was used as a case study. Microgastrine wasps are among the best represented groups of Hymenoptera in BOLD, and over 20K sequences have been already released and are publicly available (e.g., Smith et al. 2012). Microgastrinae is also one of the largest and most important groups of caterpillar parasitoids, with great importance in biological control programs worldwide (e.g., Whitfield 1997). 


\section{Materials and methods}

All specimens used for this study are deposited in the collections of the Biodiversity Institute of Ontario, University of Guelph (BIO), or the Canadian National Collection of Insects, Ottawa (CNC). They represent dozens of collecting events and different research projects carried out by those institutions. Most of the specimens rendered partial or full DNA barcodes (information can be retrieved using the following public DOI: http:// dx.doi.org/10.5883/DS-NEAMICRO), and many have associated sequences, images and/ or data from their labels in collections (Suppl. materials 1, 2, 3, 4).

We selected a sample of 630 specimens of parasitoid wasps, representing ten species and five genera of Microgastrinae (Hymenoptera: Braconidae) from the Nearctic region (Canada and the United States). The identity of the species was confirmed by JFT against authenticated material deposited in the CNC.

We downloaded the "Data Spreadsheet" associated with those records, including the worksheets "Collection Data" and "Specimens Details" (for a detailed explanation consult the "BOLD Print Handbook for BOLD v3", freely available online at http:// www.boldsystems.org/libhtml_v3/static/BOLD_Handbook_Oct2013.pdf).

The Excel file downloaded from BOLD (Suppl. material 1) was then adapted to the template provided by BDJ (a DarwinCore Compliant Excel file). Fields that were of interest for this study (e.g., locality data, collecting date, collectors, institution storing the specimens) were pasted to the corresponding columns in the BDJ template (Suppl. material 2), and from there directly imported into the Pensoft Writing Tool (the tool used to produce manuscripts for consequent submission to the BDJ). The information was uploaded as part of "Materials" for every individual species included in this study.

We compared the known distributions of the species (using mostly Fernandez-Triana 2010, Fernandez-Triana et al. 2014, Whitfield 2006, Yu et al. 2012, Fernández-Triana and Huber 2010) against the information stored in BOLD. In doing so, a significant number of new records (of locality, provinces, territories and states) were uncovered, which are detailed and discussed for every individual species under the section "Notes". The distribution records of the specimens involved in this study (Suppl. material 5) were mapped using SimpleMappr (http://www.simplemappr.net).

The ten species analyzed here represented 13 Barcode Index Numbers (BINS), sensu Ratnasingham and Hebert (2013). In three cases, single names were found to contain multiple clusters of COI variation (Suppl. materials 4,5 ). Since such variation has often been ascribed to cryptic or hidden species we examine each case under the section "Notes" for those particular species.

A few specimens discussed in this paper did not render any DNA and thus do not have any associated sequence in BOLD. However, their ancillary data (e.g., collecting date, locality, etc.) was still available and thus is presented below. 
When discussing the distribution of species, states of the United States and provinces/ territories of Canada are abbreviated with acronyms consisting of two capital letters, following Canada Post standards (http://www.canadapost.ca/tools/pg/manual/PGaddresse.asp).

\section{Species List}

\section{Apanteles carpatus (Say, 1836)}

\section{Materials}

a. country: Canada; stateProvince: Ontario; county: Ottawa; locality: Ottawa city garden; verbatimLatitude: 45.356; verbatimLongitude: -75.707; verbatimEventDate: 19-Sep-2007; lifeStage: Adult; catalogNumber: CAM0023; recordedBy: H. Goulet; otherCatalogNumbers: $\mathrm{CNCHZ213-09;} \mathrm{identifiedBy:} \mathrm{Jose} \mathrm{Fernandez-Triana;} \mathrm{institutionID:}$ Canadian National Collection of Insects, Arachnids and Nematodes

b. country: Canada; stateProvince: Ontario; county: Leeds and Grenville; municipality: Elizabethtown-Kitley; locality: 4452 Rowsome Rd., Elizabethtown; verbatimElevation: 112; verbatimLatitude: 44.621; verbatimLongitude: -75.773; verbatimEventDate: 17Jul-2010; lifeStage: Adult; catalogNumber: BIOUG01088-A03; recordedBy: James Sones; otherCatalogNumbers: JSHYO003-11; identifiedBy: Angela Telfer; institutionID: Biodiversity Institute of Ontario

c. country: Canada; stateProvince: Ontario; county: Ottawa; locality: Ottawa city garden; verbatimLatitude: 45.356; verbatimLongitude: -75.707; verbatimEventDate: 10-Aug-2007; lifeStage: Adult; catalogNumber: CAM0036; recordedBy: H. Goulet; otherCatalogNumbers: CNCHZ226-09; identifiedBy: Jose Fernandez-Triana; institutionID: Canadian National Collection of Insects, Arachnids and Nematodes

d. country: Canada; stateProvince: Ontario; county: Essex County; locality: Ruthven; verbatimEventDate: 18-Jul-1947; sex: Female; lifeStage: Adult; catalogNumber: MIC 000034; recordedBy: Dom. Par. Lab.; otherCatalogNumbers: MHABR034-09; identifiedBy: Jose Fernandez-Triana; institutionID: Canadian National Collection of Insects, Arachnids and Nematodes

e. $\quad$ country: United States; stateProvince: North Carolina; county: Bertie County; locality: near Cahaba; verbatimEventDate: 02-Jun-1976; sex: Male; lifeStage: Adult; catalogNumber: MIC 000041; recordedBy: N. C. D. A; otherCatalogNumbers: MHABR041-09; identifiedBy: Jose Fernandez-Triana; institutionID: Canadian National Collection of Insects, Arachnids and Nematodes

f. country: Canada; stateProvince: Ontario; locality: Vineland; verbatimEventDate: 17Aug-1974; sex: Female; lifeStage: Adult; catalogNumber: MIC 000036; recordedBy: Phillips; otherCatalogNumbers: MHABR036-09; identifiedBy: Jose Fernandez-Triana; institutionID: Canadian National Collection of Insects, Arachnids and Nematodes

g. country: Canada; stateProvince: Ontario; county: Leeds and Grenville; municipality: Elizabethtown-Kitley; locality: 4452 Rowsome Rd., Elizabethtown; verbatimElevation: 111; verbatimLatitude: 44.618; verbatimLongitude: -75.775 ; verbatimEventDate: 18 Aug-2011; lifeStage: Adult; catalogNumber: BIOUG01631-B09; recordedBy: James Sones; otherCatalogNumbers: JSAUG1452-11; identifiedBy: Angela Telfer; institutionID: Biodiversity Institute of Ontario 
h. country: Canada; stateProvince: Ontario; county: Ottawa; locality: Ottawa city garden; verbatimLatitude: 45.356; verbatimLongitude: -75.707; verbatimEventDate: 08-Sep-2007; lifeStage: Adult; catalogNumber: CAM0104; recordedBy: H. Goulet; otherCatalogNumbers: CNCHZ294-09; identifiedBy: Jose Fernandez-Triana; institutionID: Canadian National Collection of Insects, Arachnids and Nematodes

i. country: Canada; stateProvince: Ontario; county: Ottawa; locality: Ottawa; verbatimEventDate: 15-Sep-1955; sex: Female; lifeStage: Adult; catalogNumber: MIC 000035; recordedBy: J. Vockeroth; otherCatalogNumbers: MHABR035-09; identifiedBy: Jose Fernandez-Triana; institutionID: Canadian National Collection of Insects, Arachnids and Nematodes

j. country: United States; stateProvince: North Carolina; county: Bertie County; locality: near Cahaba; verbatimEventDate: 02-Jun-1976; lifeStage: Adult; catalogNumber: CNCHYM 00081; recordedBy: N. C. D. A; otherCatalogNumbers: HYCND1790-11; identifiedBy: Jose Fernandez-Triana; institutionID: Canadian National Collection of Insects, Arachnids and Nematodes

k. country: Canada; stateProvince: Ontario; county: Ottawa; locality: Ottawa city garden; verbatimLatitude: 45.356; verbatimLongitude: -75.707; verbatimEventDate: 30-Jul-2007; lifeStage: Adult; catalogNumber: CAM0117; recordedBy: H. Goulet;

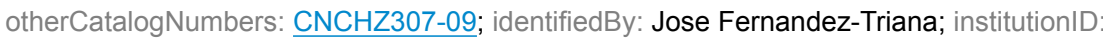
Canadian National Collection of Insects, Arachnids and Nematodes

I. country: Canada; stateProvince: Ontario; county: Ottawa; locality: Ottawa city garden; verbatimLatitude: 45.356; verbatimLongitude: -75.707; verbatimEventDate: 13-Jul-2007; lifeStage: Adult; catalogNumber: CAM0021; recordedBy: H. Goulet; otherCatalogNumbers: $\mathrm{CNCHZ211-09}$; identifiedBy: Jose Fernandez-Triana; institutionID: Canadian National Collection of Insects, Arachnids and Nematodes

m. country: Canada; stateProvince: Ontario; county: Ottawa; locality: Ottawa city garden; verbatimLatitude: 45.356; verbatimLongitude: -75.707; verbatimEventDate: 23-Jul-2007; lifeStage: Adult; catalogNumber: CAM0046; recordedBy: H. Goulet; otherCatalogNumbers: $\mathrm{CNCHZ236-09}$; identifiedBy: Jose Fernandez-Triana; institutionID: Canadian National Collection of Insects, Arachnids and Nematodes

n. country: United States; stateProvince: North Carolina; county: Bertie County; locality: near Cahaba; verbatimEventDate: 02-Jun-1976; sex: Male; lifeStage: Adult; catalogNumber: MIC 000040; recordedBy: N. C. D. A; otherCatalogNumbers: MHABR040-09; identifiedBy: Jose Fernandez-Triana; institutionID: Canadian National Collection of Insects, Arachnids and Nematodes

o. country: Canada; stateProvince: Ontario; county: Ottawa; locality: Ottawa city garden; verbatimLatitude: 45.356; verbatimLongitude: -75.707; verbatimEventDate: 23-Jul-2007; lifeStage: Adult; catalogNumber: CAM0053; recordedBy: H. Goulet; otherCatalogNumbers: CNCHZ243-09; identifiedBy: Jose Fernandez-Triana; institutionID: Canadian National Collection of Insects, Arachnids and Nematodes

p. country: Canada; stateProvince: Ontario; county: Ottawa; locality: Ottawa city garden; verbatimLatitude: 45.356; verbatimLongitude: -75.707; verbatimEventDate: 13-Jul-2007; lifeStage: Adult; catalogNumber: CAM0063; recordedBy: H. Goulet;

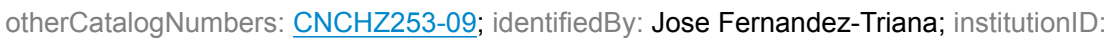
Canadian National Collection of Insects, Arachnids and Nematodes

q. country: Canada; stateProvince: Ontario; county: Ottawa; locality: Ottawa city garden; verbatimLatitude: 45.356; verbatimLongitude: -75.707; verbatimEventDate: 13-Jul-2007; lifeStage: Adult; catalogNumber: CAM0020; recordedBy: H. Goulet; otherCatalogNumbers: CNCHZ210-09; identifiedBy: Jose Fernandez-Triana; institutionID: Canadian National Collection of Insects, Arachnids and Nematodes 
r. country: Canada; stateProvince: Ontario; county: Ottawa; locality: Ottawa city garden; verbatimLatitude: 45.356; verbatimLongitude: -75.707; verbatimEventDate: 13-Jul-2007; lifeStage: Adult; catalogNumber: CAM0058; recordedBy: H. Goulet; otherCatalogNumbers: CNCHZ248-09; identifiedBy: Jose Fernandez-Triana; institutionID: Canadian National Collection of Insects, Arachnids and Nematodes

s. $\quad$ country: Canada; stateProvince: Ontario; county: Ottawa; locality: Ottawa city garden; verbatimLatitude: 45.356; verbatimLongitude: -75.707; verbatimEventDate: 30-Jul-2007; lifeStage: Adult; catalogNumber: CAM0026; recordedBy: H. Goulet; otherCatalogNumbers: $\underline{\mathrm{CNCHZ216-09}}$; identifiedBy: Jose Fernandez-Triana; institutionID: Canadian National Collection of Insects, Arachnids and Nematodes

t. country: Canada; stateProvince: Ontario; county: Ottawa; locality: Ottawa city garden; verbatimLatitude: 45.356; verbatimLongitude: -75.707; verbatimEventDate: 23-Jul-2007; lifeStage: Adult; catalogNumber: CAM0049; recordedBy: H. Goulet; otherCatalogNumbers: CNCHZ239-09; identifiedBy: Jose Fernandez-Triana; institutionID: Canadian National Collection of Insects, Arachnids and Nematodes

u. $\quad$ country: Canada; stateProvince: New Brunswick; county: York County; verbatimEventDate: 21-Oct-1981; sex: Female; lifeStage: Adult; catalogNumber: MIC 000037; recordedBy: D. Finnamore; otherCatalogNumbers: MHABR037-09; identifiedBy: Jose Fernandez-Triana; institutionID: Canadian National Collection of Insects, Arachnids and Nematodes

v. country: Canada; stateProvince: Ontario; county: Ottawa; locality: Ottawa; verbatimEventDate: 08-May-1928; sex: Female; lifeStage: Adult; catalogNumber: MIC 000033; recordedBy: C. Twinn; otherCatalogNumbers: MHABR033-09; identifiedBy: Jose Fernandez-Triana; institutionID: Canadian National Collection of Insects, Arachnids and Nematodes

w. country: Canada; stateProvince: British Columbia; locality: Aldergrove, on a poultry barn; verbatimEventDate: 15-Jul-1978; sex: Female; lifeStage: Adult; catalogNumber: MIC 000038; otherCatalogNumbers: MHABR038-09; identifiedBy: Jose Fernandez-Triana; institutionID: Canadian National Collection of Insects, Arachnids and Nematodes

x. country: Canada; stateProvince: Ontario; county: Ottawa; locality: Ottawa city garden; verbatimLatitude: 45.356; verbatimLongitude: -75.707; verbatimEventDate: 10-Aug-2007; lifeStage: Adult; catalogNumber: CAM0042; recordedBy: H. Goulet; otherCatalogNumbers: CNCHZ232-09; identifiedBy: Jose Fernandez-Triana; institutionID: Canadian National Collection of Insects, Arachnids and Nematodes

Notes: A cosmopolitan species (Yu et al. 2012), it was recorded from three provinces in Canada (BC, NB, ON) by Fernandez-Triana (2010), but no locality records were provided at the time. Here we present new details about the distribution of this species in Canada, based on examined specimens from the BIO and CNC collections. Data for those specimens was extracted from BOLD.

\section{Apanteles conanchetorum Viereck, 1917}

\section{Materials}

a. country: United States; stateProvince: North Carolina; county: Orange County; locality: Chapel Hill; verbatimEventDate: 14-Jul-1976; lifeStage: Adult; catalogNumber: CNCHYM 00088; recordedBy: N. C. D. A; otherCatalogNumbers: HYCND1797-11; identifiedBy: Jose Fernandez-Triana; institutionID: Canadian National Collection of Insects, Arachnids and Nematodes 
b. country: Canada; stateProvince: Newfoundland and Labrador; locality: St-Andrew`s; verbatimLatitude: 47.793; verbatimLongitude: -59.233; verbatimEventDate: 18-Jul-2008; lifeStage: Adult; catalogNumber: MIC 000908; recordedBy: H. Goulet, A. Badiss, C. Boudeault; otherCatalogNumbers: CNCHZ1100-09; identifiedBy: Jose Fernandez-Triana; institutionID: Canadian National Collection of Insects, Arachnids and Nematodes

c. country: Canada; stateProvince: Quebec; county: Forillon National Park; municipality: trail off of park compound (i.e. operation centre), 1501 boul. Forillon; locality: mixed forest (spruce, poplar); verbatimElevation: 135; verbatimLatitude: 48.857; verbatimLongitude: -64.376; verbatimEventDate: 18-Jul-2013; lifeStage: Adult; catalogNumber: BIOUG10353-H05; recordedBy: F.Tremblay; otherCatalogNumbers: CNFNF286-14; institutionID: Biodiversity Institute of Ontario

d. country: Canada; stateProvince: Newfoundland and Labrador; locality: St-Andrew's; verbatimLatitude: 47.793; verbatimLongitude: -59.233; verbatimEventDate: 18-Jul-2008; lifeStage: Adult; catalogNumber: MIC 000905; recordedBy: H. Goulet, A. Badiss, C. Boudeault; otherCatalogNumbers: $\mathrm{CNCHZ1097-09;}$; identifiedBy: Jose Fernandez-Triana; institutionID: Canadian National Collection of Insects, Arachnids and Nematodes

e. country: Canada; stateProvince: Ontario; county: Haldimond-Dunn Townline; municipality: Windy Bluff; verbatimElevation: 179; verbatimLatitude: 42.861; verbatimLongitude: -79.703; verbatimEventDate: 19-Sep-2010; lifeStage: Adult; catalog Number: BIOUG00989-E12; recordedBy: Alex Smith; otherCatalogNumbers: ASGLF313-11; identifiedBy: Angela Telfer; institutionID: Research Collection of M. Alex Smith

f. country: Canada; stateProvince: Ontario; county: Leeds and Grenville; municipality: Elizabethtown-Kitley; locality: 4452 Rowsome Rd., Elizabethtown; verbatimElevation: 112; verbatimLatitude: 44.621; verbatimLongitude: -75.773; verbatimEventDate: 03Aug-2010; lifeStage: Adult; catalogNumber: BIOUG01252-E11; recordedBy: James Sones; otherCatalogNumbers: JSHYO534-11; identifiedBy: BOLD ID Engine; institutionID: Biodiversity Institute of Ontario

g. country: Canada; stateProvince: Alberta; county: Waterton Lakes NP; municipality: Foothills Parkland Region; verbatimElevation: 1338; verbatimLatitude: 49.083; verbatimLongitude: -113.876; verbatimEventDate: 19-Jul-2012; lifeStage: Adult; catalogNumber: BIOUG07019-B11; recordedBy: Cyndi Smith; otherCatalogNumbers: CNWLM799-13; identifiedBy: Angela Telfer; institutionID: Biodiversity Institute of Ontario

h. country: Canada; stateProvince: Quebec; county: Forillon National Park; municipality: trail off of park compound (i.e. operation centre), 1501 boul. Forillon; locality: mixed forest (spruce, poplar); verbatimElevation: 135; verbatimLatitude: 48.857; verbatimLongitude: -64.376; verbatimEventDate: 22-Jul-2013; lifeStage: Adult; catalogNumber: BIOUG10403-A09; recordedBy: F.Tremblay; otherCatalogNumbers: CNFNF871-14; institutionID: Biodiversity Institute of Ontario

i. country: United States; stateProvince: Kansas; locality: Manhattan; verbatimEventDate: 15-Aug-1945; sex: Female; lifeStage: Adult; catalogNumber: MIC 000063; otherCatalogNumbers: MHABR063-09; identifiedBy: Jose Fernandez-Triana; institutionID: Canadian National Collection of Insects, Arachnids and Nematodes

j. country: Canada; stateProvince: Quebec; county: Forillon National Park; municipality: trail off of park compound (i.e. operation centre), 1501 boul. Forillon; locality: mixed forest (spruce, poplar); verbatimElevation: 135; verbatimLatitude: 48.857; verbatimLongitude: -64.376; verbatimEventDate: 18-Jul-2013; lifeStage: Adult; catalogNumber: BIOUG10360-E02; recordedBy: F.Tremblay; otherCatalogNumbers: CNFNF627-14; institutionID: Biodiversity Institute of Ontario

k. country: Canada; stateProvince: Quebec; county: Forillon National Park; municipality: trail off of park compound (i.e. operation centre), 1501 boul. Forillon; locality: mixed forest 
(spruce, poplar); verbatimElevation: 135; verbatimLatitude: 48.857; verbatimLongitude: -64.376; verbatimEventDate: 18-Jul-2013; lifeStage: Adult; catalogNumber: BIOUG10358-C12; recordedBy: F.Tremblay; otherCatalogNumbers: CNFNF423-14; institutionID: Biodiversity Institute of Ontario

I. country: Canada; stateProvince: Ontario; county: Haldimond-Dunn Townline; municipality: Windy Bluff; verbatimElevation: 179; verbatimLatitude: 42.861; verbatimLongitude: -79.703; verbatimEventDate: 19-Sep-2010; lifeStage: Adult; catalogNumber: BIOUG00989-F01; recordedBy: Alex Smith; otherCatalogNumbers: ASGLF314-11; identifiedBy: Angela Telfer; institutionID: Research Collection of M. Alex Smith

m. country: Canada; stateProvince: Quebec; county: Forillon National Park; municipality: trail off of park compound (i.e. operation centre), 1501 boul. Forillon; locality: mixed forest (spruce, poplar); verbatimElevation: 135; verbatimLatitude: 48.857; verbatimLongitude: -64.376; verbatimEventDate: 15-Jul-2013; lifeStage: Adult; catalog Number: BIOUG11905-C04; recordedBy: F.Tremblay; otherCatalogNumbers: CNFNR2363-14; identifiedBy: Angela Telfer; institutionID: Biodiversity Institute of Ontario

n. country: Canada; stateProvince: Newfoundland and Labrador; locality: Cap St-George, fallow field; verbatimLatitude: 48.466; verbatimLongitude: -59.260 ; verbatimEventDate: 11-Jul-2008; lifeStage: Adult; catalogNumber: MIC 000902; recordedBy: H. Goulet, A.

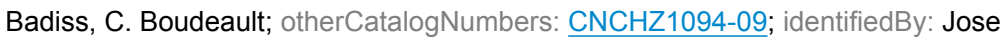
Fernandez-Triana; institutionID: Canadian National Collection of Insects, Arachnids and Nematodes

o. $\quad$ country: Canada; stateProvince: Nova Scotia; county: Queens County; locality: White Pt. Beach; verbatimEventDate: 15-Aug-1935; sex: Female; lifeStage: Adult; catalogNumber: MIC 000058; recordedBy: J. McDunnough; otherCatalogNumbers: MHABR058-09; identifiedBy: Jose Fernandez-Triana; institutionID: Canadian National Collection of Insects, Arachnids and Nematodes

p. $\quad$ country: Canada; stateProvince: Ontario; county: Ottawa; locality: Ottawa city, Slack Road; verbatimEventDate: 05-Oct-2007; lifeStage: Adult; catalogNumber: CAM0096; recordedBy: L. Masner; otherCatalogNumbers: CNCHZ286-09; identifiedBy: Jose Fernandez-Triana; institutionID: Canadian National Collection of Insects, Arachnids and Nematodes

q. country: Canada; stateProvince: Quebec; county: Forillon National Park; municipality: trail off of park compound (i.e. operation centre), 1501 boul. Forillon; locality: mixed forest (spruce, poplar); verbatimElevation: 135; verbatimLatitude: 48.857 ; verbatimLongitude: -64.376; verbatimEventDate: 15-Jul-2013; lifeStage: Adult; catalogNumber:

BIOUG11907-E10; recordedBy: F.Tremblay; otherCatalogNumbers: CNFNR2583-14; identifiedBy: Angela Telfer; institutionID: Biodiversity Institute of Ontario

r. country: Canada; stateProvince: Ontario; county: Ottawa; locality: Ottwa city garden;

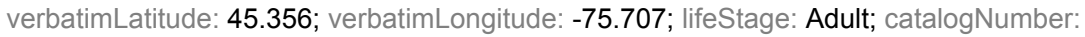
CAM1013; recordedBy: H. Goulet; otherCatalogNumbers: CNCHX143-09; identifiedBy: Jose Fernandez-Triana; institutionID: Canadian National Collection of Insects, Arachnids and Nematodes

s. $\quad$ country: Canada; stateProvince: Ontario; county: Leeds and Grenville; municipality: Elizabethtown-Kitley; locality: 4452 Rowsome Rd., Elizabethtown; verbatimElevation: 111; verbatimLatitude: 44.618; verbatimLongitude: $\mathbf{7 5 . 7 7 5}$; verbatimEventDate: $14-$ Sep-2011; lifeStage: Adult; catalogNumber: BIOUG01631-H02; recordedBy: James Sones; otherCatalogNumbers: JSSEP761-11; identifiedBy: BOLD ID Engine; institutionID: Biodiversity Institute of Ontario

t. country: United States; stateProvince: North Carolina; county: Davie County; locality: near Mocksvile; verbatimEventDate: 04-Oct-1976; lifeStage: Adult; catalogNumber: CNCHYM 
00089; recordedBy: N. C. D. A; otherCatalogNumbers: HYCND1798-11; identifiedBy: Jose Fernandez-Triana; institutionID: Canadian National Collection of Insects, Arachnids and Nematodes

u. $\quad$ country: Canada; stateProvince: Alberta; county: Waterton Lakes NP; municipality: Foothills Parkland Region; verbatimElevation: 1338; verbatimLatitude: 49.083; verbatimLongitude: -113.876; verbatimEventDate: 26-Jul-2012; lifeStage: Adult; catalogNumber: BIOUG04245-B10; recordedBy: Jarret Hardisty; otherCatalogNumbers: CNWLF652-12; institutionID: Biodiversity Institute of Ontario

v. country: Canada; stateProvince: Ontario; county: Haldimond-Dunn Townline; municipality: Windy Bluff; verbatimElevation: 179; verbatimLatitude: 42.861; verbatimLongitude: -79.703; verbatimEventDate: 19-Sep-2010; lifeStage: Adult; catalogNumber: BIOUG00989-E09; recordedBy: Alex Smith; otherCatalogNumbers: ASGLF310-11; identifiedBy: Angela Telfer; institutionID: Research Collection of M. Alex Smith

w. country: Canada; stateProvince: Quebec; county: Forillon National Park; municipality: trail off of park compound (i.e. operation centre), 1501 boul. Forillon; locality: mixed forest (spruce, poplar); verbatimElevation: 135; verbatimLatitude: 48.857; verbatimLongitude: -64.376; verbatimEventDate: 15-Jul-2013; lifeStage: Adult; catalogNumber: BIOUG11904-F02; recordedBy: F.Tremblay; otherCatalogNumbers: CNFNR2302-14; identifiedBy: Angela Telfer; institutionID: Biodiversity Institute of Ontario

x. country: Canada; stateProvince: Saskatchewan; county: Prince Albert NP; municipality:

Red Deer Trl. Red Loop; verbatimElevation: 578; verbatimLatitude: 53.907; verbatimLongitude: -106.075; verbatimEventDate: 14-Aug-2010; lifeStage: Adult; catalogNumber: 10BBCHY-2243; recordedBy: BIObus 2010; otherCatalogNumbers: BBHYK285-10; identifiedBy: Jose Fernandez-Triana; institutionID: Biodiversity Institute of Ontario

y. country: Canada; stateProvince: Ontario; locality: Woodlawn; verbatimEventDate: 06Aug-2008; lifeStage: Adult; catalogNumber: MIC 000600; recordedBy: L. Masner;

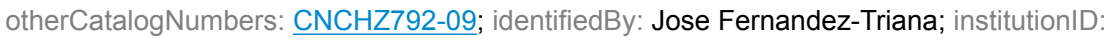
Canadian National Collection of Insects, Arachnids and Nematodes

z. country: Canada; stateProvince: Ontario; county: Point Pelee NP; municipality: 15km SE of Leamington; locality: DeLaurier Marsh and Thicket Trail; verbatimElevation: 181; verbatimLatitude: 41.949; verbatimLongitude: -82.521; verbatimEventDate: 28-Jun-2010; lifeStage: Adult; catalogNumber: PCPP10-0759; recordedBy: T.F.Mitterboeck, C.Vandermeer, V.Junea, C.Sobel; otherCatalogNumbers: BBHYN214-10; identifiedBy: Jose Fernandez-Triana; institutionID: Biodiversity Institute of Ontario

aa. country: Canada; stateProvince: Quebec; county: Forillon National Park; municipality: trail off of park compound (i.e. operation centre), 1501 boul. Forillon; locality: mixed forest (spruce, poplar); verbatimElevation: 135; verbatimLatitude: 48.857; verbatimLongitude: -64.376; verbatimEventDate: 15-Jul-2013; lifeStage: Adult; catalogNumber: BIOUG11909-G05; recordedBy: F.Tremblay; otherCatalogNumbers: CNFNR2792-14; identifiedBy: Angela Telfer; institutionID: Biodiversity Institute of Ontario

ab. country: Canada; stateProvince: Ontario; county: Haldimond-Dunn Townline; municipality: Windy Bluff; verbatimElevation: 179; verbatimLatitude: 42.861; verbatimLongitude: -79.703; verbatimEventDate: 23-Oct-2010; lifeStage: Adult; catalogNumber: BIOUG00991-A05; recordedBy: Alex Smith; otherCatalogNumbers: ASGLF353-11; identifiedBy: Angela Telfer; institutionID: Research Collection of M. Alex Smith

ac. country: Canada; stateProvince: Ontario; county: Haldimond-Dunn Townline; municipality: Windy Bluff; verbatimElevation: 179; verbatimLatitude: 42.861; verbatimLongitude: -79.703; verbatimEventDate: 21-Nov-2010; lifeStage: Adult; catalogNumber: 
BIOUG00914-E04; recordedBy: Alex Smith; otherCatalogNumbers: ASGLF115-11; identifiedBy: Angela Telfer; institutionID: Research Collection of M. Alex Smith

ad. country: Canada; stateProvince: Ontario; county: Haldimond-Dunn Townline; municipality: Windy Bluff; verbatimElevation: 179; verbatimLatitude: 42.861; verbatimLongitude: -79.703; verbatimEventDate: 21-Nov-2010; lifeStage: Adult; catalog Number: BIOUG00914-E03; recordedBy: Alex Smith; otherCatalogNumbers: ASGLF114-11; identifiedBy: Angela Telfer; institutionID: Research Collection of M. Alex Smith

ae. $\quad$ country: Canada; stateProvince: Prince Edward Island; county: Prince Edward Island National Park; municipality: Woodland Trail / Long Point; verbatimElevation: 6; verbatimLatitude: 46.412; verbatimLongitude: -63.085; verbatimEventDate: 17-Jul-2013; lifeStage: Adult; catalogNumber: BIOUG10961-E03; recordedBy: Paul Ayles; otherCatalog Numbers: CNPEP1205-14; institutionID: Biodiversity Institute of Ontario

af. country: Canada; stateProvince: Ontario; county: Campbellville; municipality: Mountsberg Conservation Area; verbatimElevation: 215; verbatimLatitude: 43.457; verbatimLongitude: -80.027; verbatimEventDate: 28-Sep-2013; lifeStage: Adult; catalogNumber: BIOUG08661-F09; recordedBy: Amy Fennell; otherCatalogNumbers: SMTPD5714-13; institutionID: Biodiversity Institute of Ontario

ag. $\quad$ country: Canada; stateProvince: Alberta; county: Waterton Lakes NP; municipality: Foothills Parkland Region; verbatimElevation: 1338; verbatimLatitude: 49.083; verbatimLongitude: -113.876; verbatimEventDate: 02-Aug-2012; lifeStage: Adult; catalogNumber: BIOUG07067-E03; recordedBy: Emma Sylvester; otherCatalogNumbers: CNWLN171-13; institutionID: Biodiversity Institute of Ontario

ah. country: Canada; stateProvince: Ontario; county: Leeds and Grenville; municipality: Elizabethtown-Kitley; locality: 4452 Rowsome Rd., Elizabethtown; verbatimElevation: 112; verbatimLatitude: 44.621; verbatimLongitude: -75.773; verbatimEventDate: 29Aug-2010; lifeStage: Adult; catalogNumber: BIOUG01280-G04; recordedBy: James Sones; otherCatalogNumbers: JSHYP027-11; identifiedBy: BOLD ID Engine; institutionID: Biodiversity Institute of Ontario

ai. country: Canada; stateProvince: Ontario; county: Ottawa; locality: Ottawa city garden; verbatimLatitude: 45.356; verbatimLongitude: -75.707; verbatimEventDate: 01-Sep-2007; lifeStage: Adult; catalogNumber: CAM0160; recordedBy: H. Goulet; otherCatalogNumbers: CNCHZ350-09; identifiedBy: Jose Fernandez-Triana; institutionID: Canadian National Collection of Insects, Arachnids and Nematodes

aj. country: Canada; stateProvince: Alberta; county: Waterton Lakes NP; municipality: Foothills Parkland Region; verbatimElevation: 1338; verbatimLatitude: 49.083; verbatimLongitude: -113.876; verbatimEventDate: 19-Jul-2012; lifeStage: Adult; catalogNumber: BIOUG06873-E09; recordedBy: Cyndi Smith; otherCatalogNumbers: CNWLM287-13; institutionID: Biodiversity Institute of Ontario

ak. country: Canada; stateProvince: Quebec; county: Forillon National Park; municipality: trail off of park compound (i.e. operation centre), 1501 boul. Forillon; locality: mixed forest (spruce, poplar); verbatimElevation: 135; verbatimLatitude: 48.857; verbatimLongitude: -64.376; verbatimEventDate: 12-Aug-2013; lifeStage: Adult; catalogNumber: BIOUG12293-G01; recordedBy: F.Tremblay; otherCatalogNumbers: CNFNT1471-14; identifiedBy: Angela Telfer; institutionID: Biodiversity Institute of Ontario

al. country: Canada; stateProvince: Quebec; county: Forillon National Park; municipality: trail off of park compound (i.e. operation centre), 1501 boul. Forillon; locality: mixed forest (spruce, poplar); verbatimElevation: 135; verbatimLatitude: 48.857; verbatimLongitude: -64.376; verbatimEventDate: 18-Jul-2013; lifeStage: Adult; catalogNumber: BIOUG10408-B06; recordedBy: F.Tremblay; otherCatalogNumbers: CNFNF1260-14; institutionID: Biodiversity Institute of Ontario 
am. country: Canada; stateProvince: Ontario; county: Haldimond-Dunn Townline; municipality: Windy Bluff; verbatimElevation: 179; verbatimLatitude: 42.861 ; verbatimLongitude: -79.703; verbatimEventDate: 19-Sep-2010; lifeStage: Adult; catalogNumber: BIOUG00989-E11; recordedBy: Alex Smith; otherCatalogNumbers: ASGLF312-11; identifiedBy: Angela Telfer; institutionID: Research Collection of M. Alex Smith

an. country: Canada; stateProvince: Quebec; county: Forillon National Park; municipality: trail off of park compound (i.e. operation centre), 1501 boul. Forillon; locality: mixed forest (spruce, poplar); verbatimElevation: 135; verbatimLatitude: 48.857; verbatimLongitude: -64.376; verbatimEventDate: 22-Jul-2013; lifeStage: Adult; catalogNumber: BIOUG10358-E06; recordedBy: F.Tremblay; otherCatalogNumbers: CNFNF441-14; institutionID: Biodiversity Institute of Ontario

ao. country: Canada; stateProvince: Ontario; county: Wellington County; municipality: Puslinch Township; locality: Concession 11/Hume Rd; verbatimElevation: 320; verbatimLatitude: 43.537; verbatimLongitude: -80.134; verbatimEventDate: 15-Oct-2010; lifeStage: Adult; catalogNumber: BIOUG00861-G06; recordedBy: Paul Hebert; otherCatalogNumbers: PHMTX962-11; identifiedBy: BOLD ID Engine; institutionID: Biodiversity Institute of Ontario

ap. country: Canada; stateProvince: Quebec; county: Forillon National Park; municipality: trail off of park compound (i.e. operation centre), 1501 boul. Forillon; locality: mixed forest (spruce, poplar); verbatimElevation: 135; verbatimLatitude: 48.857; verbatimLongitude: -64.376; verbatimEventDate: 05-Aug-2013; lifeStage: Adult; catalogNumber: BIOUG10550-D04; recordedBy: Dany Brodeau; otherCatalogNumbers: CNFNG557-14; institutionID: Biodiversity Institute of Ontario

aq. country: Canada; stateProvince: Ontario; county: Wellington County; municipality: Puslinch Township; locality: Concession 11/Hume Rd; verbatimElevation: 320; verbatimLatitude: 43.537; verbatimLongitude: -80.134; verbatimEventDate: 08-Sep-2010; lifeStage: Adult; catalogNumber: BIOUG02507-D09; recordedBy: Paul Hebert; otherCatalogNumbers: HESEP836-12; identifiedBy: BOLD ID Engine; institutionID: Biodiversity Institute of Ontario

ar. country: Canada; stateProvince: Quebec; county: Forillon National Park; municipality: trail off of park compound (i.e. operation centre), 1501 boul. Forillon; locality: mixed forest (spruce, poplar); verbatimElevation: 135; verbatimLatitude: 48.857; verbatimLongitude: -64.376; verbatimEventDate: 18-Jul-2013; lifeStage: Adult; catalogNumber: BIOUG10403-A08; recordedBy: F.Tremblay; otherCatalogNumbers: CNFNF870-14; institutionID: Biodiversity Institute of Ontario

as. country: Canada; stateProvince: Ontario; county: Haldimond-Dunn Townline; municipality: Windy Bluff; verbatimElevation: 179; verbatimLatitude: 42.861; verbatimLongitude: -79.703; verbatimEventDate: 21-Nov-2010; lifeStage: Adult; catalogNumber: BIOUG00914-E07; recordedBy: Alex Smith; otherCatalogNumbers: ASGLF118-11; identifiedBy: Angela Telfer; institutionID: Research Collection of M. Alex Smith

at. country: Canada; stateProvince: Ontario; county: Ottawa; locality: Ottawa city garden; verbatimLatitude: 45.356; verbatimLongitude: -75.707; verbatimEventDate: 01-Sep-2007; lifeStage: Adult; catalogNumber: CAM0147; recordedBy: H. Goulet;

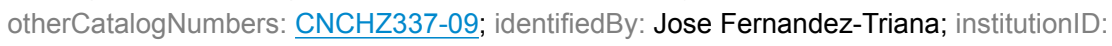
Canadian National Collection of Insects, Arachnids and Nematodes

au. country: Canada; stateProvince: Ontario; county: Guelph; municipality: 25 Division St.; verbatimElevation: 326; verbatimLatitude: 43.554; verbatimLongitude: -80.264; verbatimEventDate: 29-Oct-2010; lifeStage: Adult; catalogNumber: BIOUG00988-A09; recordedBy: Alex Smith; otherCatalogNumbers: ASGLF167-11; identifiedBy: Angela Telfer; institutionID: Research Collection of M. Alex Smith 
av. country: Canada; stateProvince: Quebec; county: Forillon National Park; municipality: trail off of park compound (i.e. operation centre), 1501 boul. Forillon; locality: mixed forest (spruce, poplar); verbatimElevation: 135; verbatimLatitude: 48.857; verbatimLongitude: -64.376; verbatimEventDate: 15-Jul-2013; lifeStage: Adult; catalogNumber: BIOUG11910-C01; recordedBy: F.Tremblay; otherCatalogNumbers: CNFNR2835-14; identifiedBy: Angela Telfer; institutionID: Biodiversity Institute of Ontario

aw. $\quad$ country: Canada; stateProvince: Alberta; county: Waterton Lakes NP; municipality: Foothills Parkland Region; verbatimElevation: 1338; verbatimLatitude: 49.083; verbatimLongitude: -113.876; verbatimEventDate: 26-Jul-2012; lifeStage: Adult; catalogNumber: BIOUG04245-A01; recordedBy: Jarret Hardisty; otherCatalogNumbers: CNWLF631-12; institutionID: Biodiversity Institute of Ontario

ax. country: Canada; stateProvince: Ontario; county: Haldimond-Dunn Townline; municipality: Windy Bluff; verbatimElevation: 179; verbatimLatitude: 42.861; verbatimLongitude; -79.703; verbatimEventDate: 21-Nov-2010; lifeStage: Adult; catalogNumber: BIOUG00914-F08; recordedBy: Alex Smith; otherCatalogNumbers: ASGLF131-11; identifiedBy: Angela Telfer; institutionID: Research Collection of M. Alex Smith

ay. $\quad$ country: Canada; stateProvince: Quebec; county: Forillon National Park; municipality: trail off of park compound (i.e. operation centre), 1501 boul. Forillon; locality: mixed forest (spruce, poplar); verbatimElevation: 135; verbatimLatitude: 48.857; verbatimLongitude: -64.376; verbatimEventDate: 05-Aug-2013; lifeStage: Adult; catalogNumber: BIOUG10661-F02; recordedBy: Dany Brodeau; otherCatalogNumbers: CNFNG1150-14; institutionID: Biodiversity Institute of Ontario

az. country: Canada; stateProvince: Quebec; county: Forillon National Park; municipality: trail off of park compound (i.e. operation centre), 1501 boul. Forillon; locality: mixed forest (spruce, poplar); verbatimElevation: 135; verbatimLatitude: 48.857 ; verbatimLongitude: -64.376; verbatimEventDate: 15-Jul-2013; lifeStage: Adult; catalogNumber: BIOUG11910-B12; recordedBy: F.Tremblay; otherCatalogNumbers: CNFNR2834-14; identifiedBy: Angela Telfer; institutionID: Biodiversity Institute of Ontario

ba. country: United States; stateProvince: Kansas; locality: Manhattan; verbatimEventDate: 15-Aug-1945; lifeStage: Adult; catalogNumber: CNCHYM 00093; otherCatalogNumbers: HYCND1803-11; identifiedBy: Jose Fernandez-Triana; institutionID: Canadian National Collection of Insects, Arachnids and Nematodes

bb. country: Canada; stateProvince: Ontario; county: Ottawa; locality: Ottawa city garden; verbatimLatitude: 45.356; verbatimLongitude: -75.707; verbatimEventDate: 19-Sep-2007; lifeStage: Adult; catalogNumber: CAM0005; recordedBy: H. Goulet;

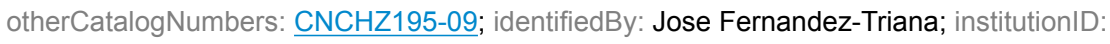
Canadian National Collection of Insects, Arachnids and Nematodes

bc. country: Canada; stateProvince: Ontario; county: Ottawa; locality: Ottawa city garden; verbatimLatitude: 45.356; verbatimLongitude: -75.707; verbatimEventDate: 10-Aug-2007; lifeStage: Adult; catalogNumber: CAM0085; recordedBy: H. Goulet; otherCatalogNumbers: CNCHZ275-09; identifiedBy: Jose Fernandez-Triana; institutionID: Canadian National Collection of Insects, Arachnids and Nematodes

bd. country: Canada; stateProvince: Ontario; county: Guelph; municipality: 25 Division St.; verbatimElevation: 326; verbatimLatitude: 43.554; verbatimLongitude: -80.264; verbatimEventDate: 29-Oct-2010; lifeStage: Adult; catalogNumber: BIOUG00989-A08; recordedBy: Alex Smith; otherCatalogNumbers: ASGLF261-11; identifiedBy: Angela Telfer; institutionID: Research Collection of M. Alex Smith

be. $\quad$ country: Canada; stateProvince: Saskatchewan; county: Prince Albert NP; municipality: Spruce River Highlands Trail; verbatimElevation: 551; verbatimLatitude: 53.712; verbatimLongitude: -106.048; verbatimEventDate: 11-Jul-2012; lifeStage: Adult; 
catalogNumber: BIOUG08192-F10; recordedBy: BIOBus 2012; otherCatalogNumbers: SSPAC3799-13; institutionID: Biodiversity Institute of Ontario

bf. country: Canada; stateProvince: Ontario; county: Leeds and Grenville; municipality: Elizabethtown-Kitley; locality: 4452 Rowsome Rd., Elizabethtown; verbatimElevation: 112; verbatimLatitude: 44.621; verbatimLongitude: -75.773; verbatimEventDate: 18Oct-2010; lifeStage: Adult; catalogNumber: BIOUG01330-D09; recordedBy: James Sones; otherCatalogNumbers: JSHYP661-12; institutionID: Biodiversity Institute of Ontario

bg. $\quad$ country: Canada; stateProvince: Quebec; county: Forillon National Park; municipality: trail off of park compound (i.e. operation centre), 1501 boul. Forillon; locality: mixed forest (spruce, poplar); verbatimElevation: 135; verbatimLatitude: 48.857 ; verbatimLongitude: -64.376; verbatimEventDate: 18-Jul-2013; lifeStage: Adult; catalogNumber: BIOUG10353-G01; recordedBy: F.Tremblay; otherCatalogNumbers: CNFNF270-14; institutionID: Biodiversity Institute of Ontario

bh. country: Canada; stateProvince: Ontario; county: Haldimond-Dunn Townline; municipality: Windy Bluff; verbatimElevation: 179; verbatimLatitude: 42.861; verbatimLongitude: -79.703; verbatimEventDate: 21-Nov-2010; lifeStage: Adult; catalogNumber: BIOUG00914-G01; recordedBy: Alex Smith; otherCatalogNumbers: ASGLF136-11; identifiedBy: Angela Telfer; institutionID: Research Collection of M. Alex Smith

bi. $\quad$ country: Canada; stateProvince: Alberta; county: Waterton Lakes NP; municipality: Foothills Parkland Region; verbatimElevation: 1338; verbatimLatitude: 49.083; verbatimLongitude: -113.876; verbatimEventDate: 19-Jul-2012; lifeStage: Adult; catalogNumber: BIOUG07067-C07; recordedBy: Cyndi Smith; otherCatalogNumbers: CNWLM1411-13; institutionID: Biodiversity Institute of Ontario

bj. $\quad$ country: Canada; stateProvince: Quebec; county: Forillon National Park; municipality: trail off of park compound (i.e. operation centre), 1501 boul. Forillon; locality: mixed forest (spruce, poplar); verbatimElevation: 135; verbatimLatitude: 48.857; verbatimLongitude: -64.376; verbatimEventDate: 30-Jul-2013; lifeStage: Adult; catalogNumber: BIOUG12037-E03; recordedBy: F.Tremblay; otherCatalogNumbers: CNFNS886-14; identifiedBy: Angela Telfer; institutionID: Biodiversity Institute of Ontario

bk. $\quad$ country: Canada; stateProvince: Ontario; county: St. Lawrence Islands National Park; locality: Grenadier Island Center; verbatimEventDate: 02-Jul-1975; sex: Female; lifeStage: Adult; catalogNumber: MIC 000061; otherCatalogNumbers: MHABR061-09; identifiedBy: Jose Fernandez-Triana; institutionID: Canadian National Collection of Insects, Arachnids and Nematodes

bl. country: Canada; stateProvince: Ontario; county: Haldimond-Dunn Townline; municipality: Windy Bluff; verbatimElevation: 179; verbatimLatitude: 42.861; verbatimLongitude: -79.703; verbatimEventDate: 19-Sep-2010; lifeStage: Adult; catalogNumber: BIOUG00989-E10; recordedBy: Alex Smith; otherCatalogNumbers: ASGLF311-11; identifiedBy: Angela Telfer; institutionID: Research Collection of M. Alex Smith

bm. country: Canada; stateProvince: Quebec; county: Forillon National Park; municipality: trail off of park compound (i.e. operation centre), 1501 boul. Forillon; locality: mixed forest (spruce, poplar); verbatimElevation: 135; verbatimLatitude: 48.857; verbatimLongitude: -64.376; verbatimEventDate: 22-Jul-2013; lifeStage: Adult; catalogNumber: BIOUG10405-G04; recordedBy: F.Tremblay; otherCatalogNumbers: CNFNF1033-14; institutionID: Biodiversity Institute of Ontario

bn. country: Canada; stateProvince: Ontario; county: Aylmer; locality: Aylmer West; verbatimEventDate: 20-Jul-1972; sex: Female; lifeStage: Adult; catalogNumber: MIC 000060; otherCatalogNumbers: MHABR060-09; identifiedBy: Jose Fernandez-Triana; institutionID: Canadian National Collection of Insects, Arachnids and Nematodes 
bo. $\quad$ country: Canada; stateProvince: Ontario; county: Ottawa; locality: Ottawa city garden; verbatimLatitude: 45.356; verbatimLongitude: -75.707; verbatimEventDate: 01-Sep-2007; lifeStage: Adult; catalogNumber: CAM0159; recordedBy: H. Goulet; otherCatalogNumbers: CNCHZ349-09; identifiedBy: Jose Fernandez-Triana; institutionID: Canadian National Collection of Insects, Arachnids and Nematodes

bp. $\quad$ country: Canada; stateProvince: Ontario; county: Rouge National Urban Park; municipality: West of Glen Rouge campground; locality: Marsh scrub along riverside; verbatimElevation: 80; verbatimLatitude: 43.804; verbatimLongitude: -79.146; verbatimEventDate: 07-Jun-2013; lifeStage: Adult; catalogNumber: BIOUG13530-A05; recordedBy: BIObus 2013; otherCatalogNumbers: SSROB2643-14; identifiedBy: Angela Telfer; institutionID: Biodiversity Institute of Ontario

bq. $\quad$ country: Canada; stateProvince: Ontario; county: Haldimond-Dunn Townline; municipality: Windy Bluff; verbatimElevation: 179; verbatimLatitude: 42.861; verbatimLongitude: -79.703; verbatimEventDate: 21-Nov-2010; lifeStage: Adult; catalogNumber: BIOUG00914-F06; recordedBy: Alex Smith; otherCatalogNumbers: ASGLF129-11; identifiedBy: Angela Telfer; institutionID: Research Collection of M. Alex Smith

br. $\quad$ country: United States; stateProvince: North Carolina; county: Avery County; locality: Linville; verbatimEventDate: 05-Oct-1976; lifeStage: Adult; catalogNumber: CNCHYM 03970; recordedBy: N. C. D. A; otherCatalogNumbers: HYCND1800-11; identifiedBy: Jose Fernandez-Triana; institutionID: Canadian National Collection of Insects, Arachnids and Nematodes

bs. $\quad$ country: Canada; stateProvince: Alberta; county: Waterton Lakes NP; municipality: Foothills Parkland Region; verbatimElevation: 1338; verbatimLatitude: 49.083; verbatimLongitude: -113.876; verbatimEventDate: 26-Jul-2012; lifeStage: Adult; catalogNumber: BIOUG04245-C02; recordedBy: Jarret Hardisty; otherCatalogNumbers: CNWLF656-12; institutionID: Biodiversity Institute of Ontario

bt. country: Canada; stateProvince: Ontario; county: Haldimond-Dunn Townline; municipality: Windy Bluff; verbatimElevation: 179; verbatimLatitude: 42.861; verbatimLongitude: -79.703; verbatimEventDate: 19-Sep-2010; lifeStage: Adult; catalogNumber: BIOUG00989-G12; recordedBy: Alex Smith; otherCatalogNumbers: ASGLF337-11; identifiedBy: Angela Telfer; institutionID: Research Collection of M. Alex Smith

bu. country: Canada; stateProvince: Ontario; county: Wellington County; municipality: Puslinch Township; locality: Concession 11/Hume Rd; verbatimElevation: 320; verbatimLatitude: 43.537; verbatimLongitude: -80.134; verbatimEventDate: 23-Sep-2010; lifeStage: Adult; catalogNumber: BIOUG01309-D01; recordedBy: Paul Hebert; otherCatalogNumbers: PHSEP1889-11; identifiedBy: BOLD ID Engine; institutionID: Biodiversity Institute of Ontario

bv. country: Canada; stateProvince: Ontario; county: Haldimond-Dunn Townline; municipality: Windy Bluff; verbatimElevation: 179; verbatimLatitude: 42.861; verbatimLongitude: -79.703; verbatimEventDate: 21-Nov-2010; lifeStage: Adult; catalogNumber: BIOUG00914-E08; recordedBy: Alex Smith; otherCatalogNumbers: ASGLF119-11; identifiedBy: Angela Telfer; institutionID: Research Collection of M. Alex Smith

bw. $\quad$ country: Canada; stateProvince: Quebec; county: Forillon National Park; municipality: trail off of park compound (i.e. operation centre), 1501 boul. Forillon; locality: mixed forest (spruce, poplar); verbatimElevation: 135; verbatimLatitude: 48.857; verbatimLongitude: -64.376; verbatimEventDate: 15-Jul-2013; lifeStage: Adult; catalogNumber: BIOUG11908-H02; recordedBy: F.Tremblay; otherCatalogNumbers: CNFNR2706-14; identifiedBy: Angela Telfer; institutionID: Biodiversity Institute of Ontario

bx. country: Canada; stateProvince: Quebec; county: Forillon National Park; municipality: trail off of park compound (i.e. operation centre), 1501 boul. Forillon; locality: mixed forest 
(spruce, poplar); verbatimElevation: 135; verbatimLatitude: 48.857; verbatimLongitude: -64.376; verbatimEventDate: 15-Jul-2013; lifeStage: Adult; catalogNumber: BIOUG11903-A01; recordedBy: F.Tremblay; otherCatalogNumbers: CNFNR2146-14; identifiedBy: Angela Telfer; institutionID: Biodiversity Institute of Ontario

by. $\quad$ country: Canada; stateProvince: Quebec; county: Forillon National Park; municipality: trail off of park compound (i.e. operation centre), 1501 boul. Forillon; locality: mixed forest (spruce, poplar); verbatimElevation: 135; verbatimLatitude: 48.857; verbatimLongitude: -64.376; verbatimEventDate: 12-Aug-2013; lifeStage: Adult; catalog Number: BIOUG12293-B06; recordedBy: F.Tremblay; otherCatalogNumbers: CNFNT1416-14; identifiedBy: Angela Telfer; institutionID: Biodiversity Institute of Ontario

bz. country: Canada; stateProvince: Quebec; county: Forillon National Park; municipality: trail off of park compound (i.e. operation centre), 1501 boul. Forillon; locality: mixed forest (spruce, poplar); verbatimElevation: 135; verbatimLatitude: 48.857; verbatimLongitude: -64.376; verbatimEventDate: 18-Jul-2013; lifeStage: Adult; catalogNumber: BIOUG10405-C03; recordedBy: F.Tremblay; otherCatalogNumbers: CNFNF984-14; institutionID: Biodiversity Institute of Ontario

ca. $\quad$ country: Canada; stateProvince: Alberta; county: Waterton Lakes NP; municipality: Foothills Parkland Region; verbatimElevation: 1338; verbatimLatitude: 49.083; verbatimLongitude: -113.876; verbatimEventDate: 19-Jul-2012; lifeStage: Adult; catalogNumber: BIOUG06873-G07; recordedBy: Cyndi Smith; otherCatalogNumbers: CNWLM309-13; institutionID: Biodiversity Institute of Ontario

cb. $\quad$ country: Canada; stateProvince: Nova Scotia; county: Queens County; locality: White Pt. Beach; verbatimEventDate: 15-Aug-1935; sex: Female; lifeStage: Adult; catalogNumber: MIC 000059; recordedBy: J. McDunnough; otherCatalogNumbers: MHABR059-09; identifiedBy: Jose Fernandez-Triana; institutionID: Canadian National Collection of Insects, Arachnids and Nematodes

cc. $\quad$ country: United States; stateProvince: North Carolina; county: Martin County; locality: near Williamston; verbatimEventDate: 16-Jun-1975; lifeStage: Adult; catalogNumber: CNCHYM 00090; recordedBy: N. C. D. A; otherCatalogNumbers: HYCND1799-11; identifiedBy: Jose Fernandez-Triana; institutionID: Canadian National Collection of Insects, Arachnids and Nematodes

cd. country: Canada; stateProvince: Ontario; county: St. Lawrence Islands National Park; locality: Thwartway Island; verbatimEventDate: 19-Jul-1976; sex: Male; lifeStage: Adult; catalogNumber: MIC 000064; otherCatalogNumbers: MHABR064-09; identifiedBy: Jose Fernandez-Triana; institutionID: Canadian National Collection of Insects, Arachnids and Nematodes

ce. country: Canada; stateProvince: Quebec; county: Forillon National Park; municipality: trail off of park compound (i.e. operation centre), 1501 boul. Forillon; locality: mixed forest (spruce, poplar); verbatimElevation: 135; verbatimLatitude: 48.857; verbatimLongitude: -64.376; verbatimEventDate: 15-Jul-2013; lifeStage: Adult; catalogNumber: BIOUG11904-C07; recordedBy: F.Tremblay; otherCatalogNumbers: CNFNR2271-14; identifiedBy: Angela Telfer; institutionID: Biodiversity Institute of Ontario

cf. country: Canada; stateProvince: Ontario; county: Haldimond-Dunn Townline; municipality: Windy Bluff; verbatimElevation: 179; verbatimLatitude: 42.861 ; verbatimLongitude: -79.703; verbatimEventDate: 12-Sep-2010; lifeStage: Adult; catalogNumber: BIOUG00991-B06; recordedBy: Alex Smith; otherCatalogNumbers: ASGLF366-11; identifiedBy: Angela Telfer; institutionID: Research Collection of M. Alex Smith

cg. $\quad$ country: Canada; stateProvince: Saskatchewan; county: Prince Albert NP; municipality: Spruce River Highlands Trail; verbatimElevation: 542; verbatimLatitude: 53.713; verbatimLongitude: -106.048; verbatimEventDate: 14-Jul-2012; lifeStage: Adult; 
catalogNumber: BIOUG09238-D12; recordedBy: BIOBus 2012; otherCatalogNumbers: SSPAC13669-13; institutionID: Biodiversity Institute of Ontario

ch. $\quad$ country: Canada; stateProvince: Quebec; county: Forillon National Park; municipality: trail off of park compound (i.e. operation centre), 1501 boul. Forillon; locality: mixed forest (spruce, poplar); verbatimElevation: 135; verbatimLatitude: 48.857 ; verbatimLongitude: -64.376; verbatimEventDate: 30-Jul-2013; lifeStage: Adult; catalogNumber: BIOUG12038-B01; recordedBy: F.Tremblay; otherCatalogNumbers: CNFNS943-14; identifiedBy: Angela Telfer; institutionID: Biodiversity Institute of Ontario

ci. country: Canada; stateProvince: Ontario; county: Guelph; municipality: 25 Division St.; verbatimElevation: 326; verbatimLatitude: 43.554; verbatimLongitude: -80.264; verbatimEventDate: 29-Oct-2010; lifeStage: Adult; catalogNumber: BIOUG00989-A07; recordedBy: Alex Smith; otherCatalogNumbers: ASGLF260-11; identifiedBy: Angela Telfer; institutionID: Research Collection of M. Alex Smith

cj. country: Canada; stateProvince: Quebec; county: Forillon National Park; municipality: trail off of park compound (i.e. operation centre), 1501 boul. Forillon; locality: mixed forest (spruce, poplar); verbatimElevation: 135; verbatimLatitude: 48.857 ; verbatimLongitude: -64.376; verbatimEventDate: 18-Jul-2013; lifeStage: Adult; catalogNumber: BIOUG10479-G03; recordedBy: F.Tremblay; otherCatalogNumbers: CNFNF2105-14; institutionID: Biodiversity Institute of Ontario

ck. country: Canada; stateProvince: Quebec; county: Forillon National Park; municipality: trail off of park compound (i.e. operation centre), 1501 boul. Forillon; locality: mixed forest (spruce, poplar); verbatimElevation: 135; verbatimLatitude: 48.857; verbatimLongitude: -64.376; verbatimEventDate: 15-Jul-2013; lifeStage: Adult; catalogNumber: BIOUG11908-G11; recordedBy: F.Tremblay; otherCatalogNumbers: CNFNR2703-14; identifiedBy: Angela Telfer; institutionID: Biodiversity Institute of Ontario

cl. country: Canada; stateProvince: Alberta; county: Waterton Lakes NP; municipality: Foothills Parkland Region; verbatimElevation: 1338; verbatimLatitude: 49.083; verbatimLongitude: -113.876; verbatimEventDate: 26-Jul-2012; lifeStage: Adult; catalogNumber: BIOUG03486-E11; recordedBy: Jarret Hardisty; otherCatalogNumbers: CNWLF272-12; institutionID: Biodiversity Institute of Ontario

cm. $\quad$ country: Canada; stateProvince: Prince Edward Island; county: Georgetown; locality: near Georgetown; verbatimElevation: 8; verbatimLatitude: 46.417; verbatimLongitude: -62.667; verbatimEventDate: 31-Jul-2005; lifeStage: Adult; catalogNumber: WMIC 0240; recordedBy: M. Sharkey; otherCatalogNumbers: $\mathrm{CNCHX240-09;} \mathrm{identifiedBy:} \mathrm{Jose}$ Fernandez-Triana; institutionID: Canadian National Collection of Insects, Arachnids and Nematodes

cn. country: Canada; stateProvince: Alberta; county: Waterton Lakes NP; municipality: Foothills Parkland Region; verbatimElevation: 1338; verbatimLatitude: 49.083; verbatimLongitude: -113.876; verbatimEventDate: 26-Jul-2012; lifeStage: Adult; catalogNumber: BIOUG04245-B04; recordedBy: Jarret Hardisty; otherCatalogNumbers: CNWLF646-12; institutionID: Biodiversity Institute of Ontario

co. $\quad$ country: United States; stateProvince: North Carolina; county: Avery County; locality: Linville; verbatimEventDate: 26-Aug-1976; sex: Female; lifeStage: Adult; catalogNumber: MIC 000062; otherCatalogNumbers: MHABR062-09; identifiedBy: Jose FernandezTriana; institutionID: Canadian National Collection of Insects, Arachnids and Nematodes

cp. country: Canada; stateProvince: Quebec; county: Forillon National Park; municipality: trail off of park compound (i.e. operation centre), 1501 boul. Forillon; locality: mixed forest (spruce, poplar); verbatimElevation: 135; verbatimLatitude: 48.857 ; verbatimLongitude: -64.376; verbatimEventDate: 15-Jul-2013; lifeStage: Adult; catalogNumber: 
BIOUG11907-B04; recordedBy: F.Tremblay; otherCatalogNumbers: CNFNR2541-14; identifiedBy: Angela Telfer; institutionID: Biodiversity Institute of Ontario

cq. country: Canada; stateProvince: Ontario; county: Leeds and Grenville; municipality: Elizabethtown-Kitley; locality: 4452 Rowsome Rd., Elizabethtown; verbatimElevation: 112; verbatimLatitude: 44.621; verbatimLongitude: -75.773 ; verbatimEventDate: $04-$ Oct-2010; lifeStage: Adult; catalogNumber: BIOUG01327-H05; recordedBy: James Sones; otherCatalogNumbers: JSHYP610-11; institutionID: Biodiversity Institute of Ontario

Notes: This species is widely distributed in central and eastern North America. It was recorded from two provinces in Canada (NS, ON) by Fernandez-Triana (2010), but no locality records were provided at the time. Here we present additional information about the distribution of this species in Canada, including five new provincial records $(A B, N L$, $\mathrm{PE}, \mathrm{QC}, \mathrm{SK}$ ), based on examined specimens from the BIO and CNC collections. Data for those specimens was extracted from BOLD.

The specimens of $A$. conanchetorum that rendered DNA barcodes comprise two BINS: BOLD:AAC5506 (eastern North America) and BOLD:AAC5507 (principally Western Canada, but some records from ON, PE) (Suppl. material 4); whether they represent two different species or not will be studied in an upcoming paper.

\section{Apanteles ensiger (Say, 1836)}

\section{Materials}

a. country: Canada; stateProvince: Ontario; county: Heron Bay; municipality: Pukaskwa National Park; verbatimElevation: 631; verbatimLatitude: 48.601; verbatimLongitude: -86.289; verbatimEventDate: 26-Aug-2013; lifeStage: Adult; catalogNumber: BIOUG11625-E04; recordedBy: Cavan Harpur; otherCatalogNumbers: CNPKJ648-14; institutionID: Biodiversity Institute of Ontario

b. country: Canada; stateProvince: Ontario; municipality: Carden Alvar; locality: North Bear; verbatimLatitude: 44.695; verbatimLongitude: -79.039; verbatimEventDate: 06-Oct-2011; lifeStage: Adult; catalogNumber: BIOUG01657-E06; recordedBy: J. Cossey; otherCatalogNumbers: NCCC149-11; institutionID: Biodiversity Institute of Ontario

c. country: Canada; stateProvince: Manitoba; county: Churchill; locality: 23 km E Churchill, Ramsay Creek; verbatimElevation: 10; verbatimLatitude: 58.731 ; verbatimLongitude: -93.780; verbatimEventDate: 02-Aug-2007; lifeStage: Adult; catalogNumber: 07PROBE-22328; recordedBy: A.Renaud; otherCatalogNumbers: ASWAV869-08; identifiedBy: Jose Fernandez-Triana; institutionID: Biodiversity Institute of Ontario

d. country: Canada; stateProvince: Nova Scotia; county: Kejimkujik National Park; locality: Jeremy`s Bay Campground, near Ampitheater off of Campfire Circle; verbatimElevation: 116; verbatimLatitude: 44.407; verbatimLongitude: -65.245; verbatimEventDate: 25 Jul-2013; lifeStage: Adult; catalogNumber: BIOUG11759-F05; recordedBy: Matt Davis; otherCatalogNumbers: CNKJF1743-14; institutionID: Biodiversity Institute of Ontario

e. country: Canada; stateProvince: Newfoundland and Labrador; county: Plum Point; locality: Plum Point, fallow field; verbatimLatitude: 51.064; verbatimLongitude: -56.982; verbatimEventDate: 16-Jul-2008; lifeStage: Adult; catalogNumber: CAM0960; recordedBy: H. Goulet, A. Badiss, C. Boudeault; otherCatalogNumbers: CNCHX090-09; identifiedBy: Jose Fernandez-Triana; institutionID: Canadian National Collection of Insects, Arachnids and Nematodes 
f. country: Canada; stateProvince: Ontario; county: Heron Bay; municipality: Pukaskwa National Park; verbatimElevation: 631; verbatimLatitude: 48.601; verbatimLongitude: -86.289; verbatimEventDate: 01-Sep-2013; lifeStage: Adult; catalogNumber: BIOUG14172-C09; recordedBy: Cavan Harpur; otherCatalogNumbers: CNPKO3073-14; identifiedBy: Angela Telfer; institutionID: Biodiversity Institute of Ontario

g. country: Canada; stateProvince: Ontario; county: Leeds and Grenville; municipality: Elizabethtown-Kitley; locality: 4452 Rowsome Rd., Elizabethtown; verbatimElevation: 111; verbatimLatitude: 44.618; verbatimLongitude: 75.775 ; verbatimEventDate: $14-$ Sep-2011; lifeStage: Adult; catalogNumber: BIOUG01631-D07; recordedBy: James Sones; otherCatalogNumbers: JSSEP718-11; identifiedBy: BOLD ID Engine; institutionID: Biodiversity Institute of Ontario

h. country: Canada; stateProvince: Ontario; county: Wellington County; municipality: Guelph; locality: Biodiversity Institute of Ontario; verbatimElevation: 335; verbatimLatitude: 43.528; verbatimLongitude: -80.229; verbatimEventDate: 27-Sep-2013; lifeStage: Adult; catalogNumber: BIOUG08683-C10; recordedBy: BIO Collections Staff; otherCatalogNumbers: SMTPD5208-13; institutionID: Biodiversity Institute of Ontario

i. country: Canada; stateProvince: Ontario; county: Heron Bay; municipality: Pukaskwa National Park; verbatimElevation: 631; verbatimLatitude: 48.601; verbatimLongitude: -86.289; verbatimEventDate: 01-Sep-2013; lifeStage: Adult; catalog Number: BIOUG14172-G04; recordedBy: Cavan Harpur; otherCatalogNumbers: CNPKO3116-14; identifiedBy: Angela Telfer; institutionID: Biodiversity Institute of Ontario

j. $\quad$ country: Canada; stateProvince: Ontario; county: Ottawa; locality: Ottawa city garden; verbatimLatitude: 45.356; verbatimLongitude: -75.707; verbatimEventDate: 19-Sep-2007; lifeStage: Adult; catalogNumber: CAM0009; recordedBy: H. Goulet;

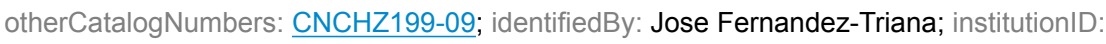
Canadian National Collection of Insects, Arachnids and Nematodes

k. country: Canada; stateProvince: Ontario; county: Wellington County; municipality: Guelph; locality: Biodiversity Institute of Ontario; verbatimElevation: 335; verbatimLatitude: 43.528; verbatimLongitude: -80.229; verbatimEventDate: 13-Jun-2013; lifeStage: Adult; catalogNumber: BIOUG08669-D07; recordedBy: BIO Collections Staff; otherCatalogNumbers: MBIOD945-13; institutionID: Biodiversity Institute of Ontario

I. country: Canada; stateProvince: Newfoundland and Labrador; county: Corner Brook; locality: Corner Brook, fallow field; verbatimLatitude: 48.956; verbatimLongitude: -57.911; verbatimEventDate: 17-Jul-2008; lifeStage: Adult; catalogNumber: CAM0940; recordedBy: H. Goulet, A. Badiss, C. Boudeault; otherCatalogNumbers: $\mathrm{CNCHX070-09;}$ identifiedBy: Jose Fernandez-Triana; institutionID: Canadian National Collection of Insects, Arachnids and Nematodes

m. country: Canada; stateProvince: Ontario; county: Rondeau Provincial Park; verbatimEventDate: 16-Jul-1972; sex: Female; lifeStage: Adult; catalogNumber: MIC 000087; otherCatalogNumbers: MHABR087-09; identifiedBy: Jose Fernandez-Triana; institutionID: Canadian National Collection of Insects, Arachnids and Nematodes

n. country: Canada; stateProvince: Ontario; county: Guelph; municipality: John F. Ross Collegiate \& Vocational Institute; locality: EQP-CLL-524; verbatimElevation: 329; verbatimLatitude: 43.562; verbatimLongitude: -80.247; verbatimEventDate: 27-Sep-2013; lifeStage: Adult; catalogNumber: BIOUG08641-D09; recordedBy: M.Neerhof, S.Lipskie; otherCatalogNumbers: SMTPD3239-13; institutionID: Biodiversity Institute of Ontario

o. country: Canada; stateProvince: Ontario; county: Wellington County; municipality: Guelph; locality: Biodiversity Institute of Ontario; verbatimElevation: 335; verbatimLatitude: 43.528; verbatimLongitude: -80.229; verbatimEventDate: 27-Sep-2013; 
lifeStage: Adult; catalogNumber: BIOUG08683-G07; recordedBy: BIO Collections Staff; otherCatalogNumbers: SMTPD5253-13; institutionID: Biodiversity Institute of Ontario

p. country: Canada; stateProvince: Ontario; county: Paris; municipality: Holy Family School; locality: EQP-CLL-560; verbatimElevation: 250; verbatimLatitude: 43.207; verbatimLongitude: -80.391; verbatimEventDate: 27-Sep-2013; lifeStage: Adult; catalogNumber: BIOUG08663-H08; recordedBy: Anne Parkhill; otherCatalogNumbers: SMTPD4869-13; institutionID: Biodiversity Institute of Ontario

q. country: Canada; stateProvince: Ontario; county: Toronto; municipality: Rouge National Urban Park, Trap 1; verbatimElevation: 136; verbatimLatitude: 43.822; verbatimLongitude: -79.190; verbatimEventDate: 27-Aug-2013; lifeStage: Adult; catalogNumber: BIOUG09765-A01; recordedBy: Lydia Attard, Kyla Greenham; otherCatalogNumbers: CNROS470-13; institutionID: Biodiversity Institute of Ontario

r. country: Canada; stateProvince: Ontario; county: Wellington County; municipality: Guelph; locality: Biodiversity Institute of Ontario; verbatimElevation: 335; verbatimLatitude: 43.528; verbatimLongitude: -80.229; verbatimEventDate: 27-Sep-2013; lifeStage: Adult; catalogNumber: BIOUG08683-C04; recordedBy: BIO Collections Staff; otherCatalogNumbers: SMTPD5202-13; institutionID: Biodiversity Institute of Ontario

s. $\quad$ country: Canada; stateProvince: Ontario; county: Heron Bay; municipality: Pukaskwa National Park; verbatimElevation: 631; verbatimLatitude: 48.601; verbatimLongitude: -86.289; verbatimEventDate: 01-Sep-2013; lifeStage: Adult; catalogNumber: BIOUG14108-F04; recordedBy: Cavan Harpur; otherCatalogNumbers: CNPKO1489-14; identifiedBy: Angela Telfer; institutionID: Biodiversity Institute of Ontario

t. country: Canada; stateProvince: Ontario; county: St. Marys; municipality: Little Falls Public School; locality: EQP-CLL-554; verbatimElevation: 305; verbatimLatitude: 43.259; verbatimLongitude: -81.142; verbatimEventDate: 27-Sep-2013; lifeStage: Adult; catalogNumber: BIOUG08647-G04; recordedBy: Lisa Cairncross; otherCatalogNumbers: SMTPD3828-13; institutionID: Biodiversity Institute of Ontario

u. country: Canada; stateProvince: Ontario; county: Wellington County; municipality: Guelph; locality: Biodiversity Institute of Ontario; verbatimElevation: 335; verbatimLatitude: 43.528; verbatimLongitude: -80.229; verbatimEventDate: 04-Oct-2013; lifeStage: Adult; catalogNumber: BIOUG09932-C07; recordedBy: BIO Collections Staff; otherCatalogNumbers: MBION508-14; institutionID: Biodiversity Institute of Ontario

v. country: Canada; stateProvince: Manitoba; locality: Gardenton; verbatimElevation: 292; verbatimLatitude: 49.079; verbatimLongitude: -96.766; verbatimEventDate: 11-Jun-2007; lifeStage: Adult; catalogNumber: HYM00001023; recordedBy: Goulet \& Boudreault; otherCatalogNumbers: ASWA309-08; identifiedBy: Jose Fernandez-Triana; institutionID: Canadian National Collection of Insects, Arachnids and Nematodes

w. country: Canada; stateProvince: Ontario; county: Georgian Bay Islands National Park; municipality: Beausoleil Island; locality: Cedar Spring Campground, mixed forest; verbatimElevation: 177; verbatimLatitude: 44.852; verbatimLongitude: -79.874; verbatimEventDate: 27-Aug-2013; lifeStage: Adult; catalogNumber: BIOUG10202-A04; recordedBy: Chris Johnstone; otherCatalogNumbers: CNGBG1411-14; institutionID: Biodiversity Institute of Ontario

x. country: Canada; stateProvince: Ontario; county: Almonte; locality: 5 km NW of Almonte, Hwy 49, Burnt Land, Alvar Prov. Park; verbatimLatitude: 45.255; verbatimLongitude: -76.140; verbatimEventDate: 29-May-2008; lifeStage: Adult; catalogNumber: CAM0342; recordedBy: Goulet \& Fernandez; otherCatalogNumbers: CNCHZ532-09; identifiedBy: Jose Fernandez-Triana; institutionID: Canadian National Collection of Insects, Arachnids and Nematodes 
y. country: Canada; stateProvince: Newfoundland and Labrador; locality: Corner Brook, fallow field; verbatimLatitude: 48.956; verbatimLongitude: -57.911 ; verbatimEventDate: 17-Jul-2008; lifeStage: Adult; catalogNumber: MIC 000903; recordedBy: H. Goulet, A. Badiss, C. Boudeault; otherCatalogNumbers: CNCHZ1095-09; identifiedBy: Jose Fernandez-Triana; institutionID: Canadian National Collection of Insects, Arachnids and Nematodes

z. country: Canada; stateProvince: Ontario; county: Heron Bay; municipality: Pukaskwa National Park; verbatimElevation: 631; verbatimLatitude: 48.601; verbatimLongitude: -86.289; verbatimEventDate: 26-Aug-2013; lifeStage: Adult; catalogNumber: BIOUG11707-A06; recordedBy: Cavan Harpur; otherCatalogNumbers: CNPKJ2122-14; institutionID: Biodiversity Institute of Ontario

aa. $\quad$ country: Canada; stateProvince: Manitoba; locality: Gardenton; verbatimElevation: 292; verbatimLatitude: 49.079; verbatimLongitude: -96.766; verbatimEventDate: 11-Jun-2007; lifeStage: Adult; catalogNumber: HYM00001022; recordedBy: Goulet \& Boudreault; otherCatalogNumbers: ASWA308-08; identifiedBy: Jose Fernandez-Triana; institutionID: Canadian National Collection of Insects, Arachnids and Nematodes

ab. country: Canada; stateProvince: Ontario; county: Thousand Islands NP; municipality: Jones Creek by Mallorytown, County Rd. 5; verbatimElevation: 117; verbatimLatitude: 44.475; verbatimLongitude: -75.865; verbatimEventDate: 12-Aug-2012; lifeStage: Adult; catalogNumber: BIOUG06823-D09; recordedBy: Thousand Islands NP;

otherCatalogNumbers: CNSLS106-13; institutionID: Biodiversity Institute of Ontario

ac. country: Canada; stateProvince: Ontario; county: St. Marys; municipality: Little Falls Public School; locality: EQP-CLL-554; verbatimElevation: 305; verbatimLatitude: 43.259; verbatimLongitude: -81.142; verbatimEventDate: 27-Sep-2013; lifeStage: Adult; catalogNumber: BIOUG08647-C01; recordedBy: Lisa Cairncross; otherCatalogNumbers: SMTPD3777-13; institutionID: Biodiversity Institute of Ontario

ad. country: Canada; stateProvince: Newfoundland and Labrador; locality: Plum Point, fallow field; verbatimLatitude: 51.064; verbatimLongitude: -56.848; verbatimEventDate: 16 Jul-2008; lifeStage: Adult; catalogNumber: MIC 000910; recordedBy: H. Goulet, A. Badiss, C. Boudeault; otherCatalogNumbers: $\mathrm{CNCHZ1102-09;} \mathrm{identifiedBy:} \mathrm{Jose}$ Fernandez-Triana; institutionID: Canadian National Collection of Insects, Arachnids and Nematodes

ae. country: Canada; stateProvince: Ontario; county: Ottawa; locality: Mixed forest near Manotick; verbatimLatitude: 45.235; verbatimLongitude: -75.624; lifeStage: Adult; catalogNumber: CAM0526; recordedBy: A. Bennett; otherCatalogNumbers: CNCHZ1146-09; identifiedBy: Jose Fernandez-Triana; institutionID: Canadian National Collection of Insects, Arachnids and Nematodes

af. country: Canada; stateProvince: Newfoundland and Labrador; county: Corner Brook; locality: Corner Brook, fallow field; verbatimLatitude: 48.956; verbatimLongitude: -57.911; verbatimEventDate: 17-Jul-2008; lifeStage: Adult; catalogNumber: CAM0962; recordedBy: H. Goulet, A. Badiss, C. Boudeault; otherCatalogNumbers: CNCHX092-09; identifiedBy: Jose Fernandez-Triana; institutionID: Canadian National Collection of Insects, Arachnids and Nematodes

ag. country: Canada; stateProvince: Prince Edward Island; locality: Blooming Point, fallow field; verbatimElevation: 6; verbatimLatitude: 46.408; verbatimLongitude: -62.951; verbatimEventDate: 23-Jul-2008; lifeStage: Adult; catalogNumber: MIC 000493; recordedBy: H. Goulet, A. Badiss, C. Boudeault; otherCatalogNumbers: $\underline{\mathrm{CNCHZ685-09}}$; identifiedBy: Jose Fernandez-Triana; institutionID: Canadian National Collection of Insects, Arachnids and Nematodes 
ah. country: Canada; stateProvince: Alberta; county: Wood Buffalo NP; municipality: Benchmark weather station; verbatimElevation: 219; verbatimLatitude: 59.561; verbatimLongitude: -112.261; verbatimEventDate: 05-Aug-2012; lifeStage: Adult; catalogNumber: BIOUG06549-D07; recordedBy: Nicole Labine; otherCatalogNumbers: CNWBH1210-13; institutionID: Biodiversity Institute of Ontario

ai. country: Canada; stateProvince: Ontario; county: Point Pelee NP; municipality: Cactus Field; verbatimElevation: 168; verbatimLatitude: 41.939; verbatimLongitude: -82.516; verbatimEventDate: 01-Jun-2012; lifeStage: Adult; catalogNumber: BIOUG03220-A01; recordedBy: Heidi Brown; otherCatalogNumbers: CNPPC821-12; institutionID: Biodiversity Institute of Ontario

aj. $\quad$ country: Canada; stateProvince: Quebec; county: Belle-Anse; locality: Belle-Anse; verbatimLatitude: 48.625; verbatimLongitude: -64.178; verbatimEventDate: 26-Jul-2008; lifeStage: Adult; catalogNumber: CAM0290; recordedBy: H. Goulet, A. Badiss, C. Boudeault; otherCatalogNumbers: $\mathrm{CNCHZ480-09;}$; identifiedBy: Jose Fernandez-Triana; institutionID: Canadian National Collection of Insects, Arachnids and Nematodes

ak. country: Canada; stateProvince: Manitoba; county: Aweme; locality: Criddle Homestead, mixed-grass prairie; verbatimLatitude: 49.706; verbatimLongitude: -99.576; verbatimEventDate: 24-Jul-2007; lifeStage: Adult; catalogNumber: MIC 000637; recordedBy: H. Goulet; otherCatalogNumbers: $\mathrm{CNCHZ829-09;} \mathrm{identifiedBy:} \mathrm{Jose}$ Fernandez-Triana; institutionID: Canadian National Collection of Insects, Arachnids and Nematodes

al. country: Canada; stateProvince: Ontario; county: Ottawa; locality: Ottawa city garden; verbatimLatitude: 45.356; verbatimLongitude: -75.707; verbatimEventDate: 30-May-2007; lifeStage: Adult; catalogNumber: CAM0011; recordedBy: H. Goulet;

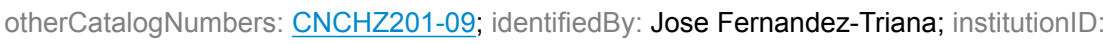
Canadian National Collection of Insects, Arachnids and Nematodes

am. Country: Canada; stateProvince: Manitoba; locality: Gardenton; verbatimElevation: 292; verbatimLatitude: 49.079; verbatimLongitude: -96.766; verbatimEventDate: 11-Jun-2007; lifeStage: Adult; catalogNumber: HYM00001027; recordedBy: Goulet \& Boudreault; otherCatalogNumbers: ASWA313-08; identifiedBy: Jose Fernandez-Triana; institutionID: Canadian National Collection of Insects, Arachnids and Nematodes

an. country: Canada; stateProvince: Ontario; county: Thousand Islands NP; municipality: Jones Creek by Mallorytown, County Rd. 5; verbatimElevation: 117; verbatimLatitude: 44.475; verbatimLongitude: -75.865; verbatimEventDate: 12-Aug-2012; lifeStage: Adult; catalogNumber: BIOUG06823-E10; recordedBy: Thousand Islands NP; otherCatalogNumbers: CNSLS119-13; institutionID: Biodiversity Institute of Ontario

ao. country: Canada; stateProvince: Newfoundland and Labrador; county: Corner Brook; locality: Corner Brook, fallow field; verbatimLatitude: 48.956; verbatimLongitude: -57.911; verbatimEventDate: 17-Jul-2008; lifeStage: Adult; catalogNumber: CAM0939; recordedBy: H. Goulet, A. Badiss, C. Boudeault; otherCatalogNumbers: CNCHX069-09; identifiedBy: Jose Fernandez-Triana; institutionID: Canadian National Collection of Insects, Arachnids and Nematodes

ap. country: Canada; stateProvince: Ontario; county: Heron Bay; municipality: Pukaskwa National Park; verbatimElevation: 631; verbatimLatitude: 48.601; verbatimLongitude: -86.289; verbatimEventDate: 26-Aug-2013; lifeStage: Adult; catalogNumber: BIOUG11771-G06; recordedBy: Cavan Harpur; otherCatalogNumbers: CNPKJ2479-14; institutionID: Biodiversity Institute of Ontario

aq. country: Canada; stateProvince: Ontario; county: Wellington County; municipality: Guelph; locality: Biodiversity Institute of Ontario; verbatimElevation: 335; verbatimLatitude: 43.528; verbatimLongitude: -80.229; verbatimEventDate: 27-Jun-2013; 
lifeStage: Adult; catalogNumber: BIOUG08748-E07; recordedBy: BIO Collections Staff; otherCatalogNumbers: MBIOE1290-13; institutionID: Biodiversity Institute of Ontario

ar. country: Canada; stateProvince: Ontario; county: Almonte; locality: 5 km NW of Almonte, Hwy 49, Burnt Land, Alvar Prov. Park; verbatimLatitude: 45.255; verbatimLongitude: -76.140; verbatimEventDate: 29-May-2008; lifeStage: Adult; catalogNumber: CAM0352;

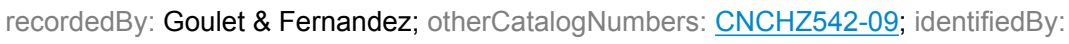
Jose Fernandez-Triana; institutionID: Canadian National Collection of Insects, Arachnids and Nematodes

as. $\quad$ country: Canada; stateProvince: Ontario; county: Leeds; locality: Leeds-Granville Co., forest; verbatimLatitude: 44.629; verbatimLongitude: -76.359; verbatimEventDate: 01 Oct-2008; lifeStage: Adult; catalogNumber: CAM0448; recordedBy: S. Peck; otherCatalogNumbers: CNCHZ638-09; identifiedBy: Jose Fernandez-Triana; institutionID: Canadian National Collection of Insects, Arachnids and Nematodes

at. country: Canada; stateProvince: Ontario; county: Wellington County; municipality: Guelph; locality: Biodiversity Institute of Ontario; verbatimElevation: 335; verbatimLatitude: 43.528; verbatimLongitude: -80.229; verbatimEventDate: 19-Sep-2013; lifeStage: Adult; catalogNumber: BIOUG09807-F11; recordedBy: BIO Collections Staff; otherCatalogNumbers: MBIOK461-14; institutionID: Biodiversity Institute of Ontario au. country: Canada; stateProvince: Alberta; county: Banff NP; municipality: Corral Creek old road; verbatimElevation: 1539; verbatimLatitude: 51.407; verbatimLongitude: -116.154 ; verbatimEventDate: 24-Jul-2012; lifeStage: Adult; catalogNumber: BIOUG06471-C10; recordedBy: BIOBus 2012; otherCatalogNumbers: SSBAF2145-13; institutionID: Biodiversity Institute of Ontario

av. $\quad$ country: United States; stateProvince: Alaska; locality: Trapper Creek, Petersville Road; verbatimElevation: 127; verbatimLatitude: 62.316; verbatimLongitude: -150.261; verbatimEventDate: 17-Jul-2009; lifeStage: Adult; catalogNumber: CNCH3194; recordedBy: Goulet \& Bouderault; otherCatalogNumbers: CNCHV264-10; identifiedBy: Jose Fernandez-Triana; institutionID: Canadian National Collection of Insects, Arachnids and Nematodes

aw. country: Canada; stateProvince: Ontario; county: Point Pelee NP; municipality: Cactus Field; verbatimElevation: 168; verbatimLatitude: 41.939; verbatimLongitude: -82.516; verbatimEventDate: 01-Jun-2012; lifeStage: Adult; catalogNumber: BIOUG03220-F05; recordedBy: Heidi Brown; otherCatalogNumbers: CNPPC885-12; institutionID: Biodiversity Institute of Ontario

ax. country: Canada; stateProvince: Quebec; county: Belle-Anse; locality: Belle-Anse; verbatimLatitude: 48.625; verbatimLongitude: -64.178; verbatimEventDate: 26-Jul-2008; lifeStage: Adult; catalogNumber: CAM0289; recordedBy: H. Goulet, A. Badiss, C.

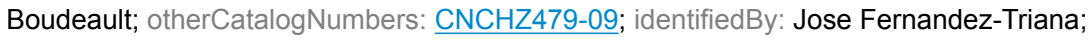
institutionID: Canadian National Collection of Insects, Arachnids and Nematodes

ay. country: Canada; stateProvince: Ontario; municipality: Carden Alvar; locality: North Bear; verbatimLatitude: 44.695; verbatimLongitude: -79.039; verbatimEventDate: 06-Oct-2011; lifeStage: Adult; catalogNumber: BIOUG01657-F10; recordedBy: J. Cossey; otherCatalogNumbers: NCCC165-11; institutionID: Biodiversity Institute of Ontario

az. country: Canada; stateProvince: Ontario; county: Almonte; locality: 5 km NW of Almonte, Hwy 49, Burnt Land, Alvar Prov. Park; verbatimLatitude: 45.255; verbatimLongitude: -76.140; verbatimEventDate: 29-May-2008; lifeStage: Adult; catalogNumber: CAM0344; recordedBy: Goulet \& Fernandez; otherCatalogNumbers: CNCHZ534-09; identifiedBy: Jose Fernandez-Triana; institutionID: Canadian National Collection of Insects, Arachnids and Nematodes 
ba. country: Canada; stateProvince: Ontario; locality: North Gower to Smith Falls, $1 \mathrm{~km} \mathrm{~N}$ of Rd 6 \& Montague Bdy Rd; verbatimLatitude: 45.033; verbatimLongitude: -75.900; verbatimEventDate: 15-Jun-2004; lifeStage: Adult; catalogNumber: HYM00001003; recordedBy: Bennet \& Barnes; otherCatalogNumbers: ASWA289-08; identifiedBy: Jose Fernandez-Triana; institutionID: Canadian National Collection of Insects, Arachnids and Nematodes

bb. $\quad$ country: Canada; stateProvince: Ontario; county: Ottawa; locality: Mixed forest near Manotick; verbatimLatitude: 45.235; verbatimLongitude: -75.624; lifeStage: Adult; catalogNumber: CAM0525; recordedBy: A. Bennett; otherCatalogNumbers: CNCHZ1145-09; identifiedBy: Jose Fernandez-Triana; institutionID: Canadian National Collection of Insects, Arachnids and Nematodes

bc. country: Canada; stateProvince: Ontario; county: Whitby; municipality: Donald A. Wilson Secondary School; locality: EQP-CLL-597; verbatimElevation: 91; verbatimLatitude: 43.894; verbatimLongitude: -78.964; verbatimEventDate: 27-Sep-2013; lifeStage: Adult; catalogNumber: BIOUG08643-F01; recordedBy: Linda Phillips; otherCatalogNumbers: SMTPD3455-13; institutionID: Biodiversity Institute of Ontario

bd. $\quad$ country: Canada; stateProvince: Quebec; locality: Gatineau Park; verbatimEventDate: 15Jun-1977; sex: Female; lifeStage: Adult; catalogNumber: MIC 000090; recordedBy: L. Masner; otherCatalogNumbers: MHABR090-09; identifiedBy: Jose Fernandez-Triana; institutionID: Canadian National Collection of Insects, Arachnids and Nematodes

be. country: Canada; stateProvince: Manitoba; county: Churchill; municipality: 10 km ESE Churchill, Launch Road near A-frame; locality: Launch road Fen; verbatimLatitude: 58.754; verbatimLongitude: -93.998; verbatimEventDate: 31-Jul-2010; lifeStage: Adult; catalogNumber: BIOUG00761-A09; recordedBy: Brandon Laforest; otherCatalogNumbers: JBHCI617-11; identifiedBy: Jose Fernandez-Triana; institutionID: Biodiversity Institute of Ontario

bf. country: Canada; stateProvince: Manitoba; county: Churchill; locality: unspecified locality; verbatimLatitude: 58.740; verbatimLongitude: -93.820; verbatimEventDate: 17-Aug-2006; lifeStage: Adult; catalogNumber: HYM00000665.2; otherCatalogNumbers: ASWAY519-08 ; identifiedBy: Jose Fernandez-Triana; institutionID: Canadian National Collection of Insects, Arachnids and Nematodes

bg. country: Canada; stateProvince: Ontario; county: Wellington County; municipality: Guelph; locality: Biodiversity Institute of Ontario; verbatimElevation: 335; verbatimLatitude: 43.528; verbatimLongitude: -80.229; verbatimEventDate: 27-Sep-2013; lifeStage: Adult; catalogNumber: BIOUG09925-E09; recordedBy: BIO Collections Staff; otherCatalogNumbers: MBIOL366-14; institutionID: Biodiversity Institute of Ontario

bh. country: Canada; stateProvince: Quebec; locality: Knowlton; verbatimEventDate: 24Aug-1929; sex: Female; lifeStage: Adult; catalogNumber: MIC 000084; recordedBy: L. Milne; otherCatalogNumbers: MHABR084-09; identifiedBy: Jose Fernandez-Triana; institutionID: Canadian National Collection of Insects, Arachnids and Nematodes

bi. country: Canada; stateProvince: Ontario; municipality: Carden Alvar; locality: Prairie Smoke; verbatimLatitude: 44.645; verbatimLongitude: -79.095; verbatimEventDate: 06Oct-2011; lifeStage: Adult; catalogNumber: BIOUG01572-H03; recordedBy: J. Cossey; otherCatalogNumbers: NCCC087-11; institutionID: Biodiversity Institute of Ontario

bj. country: Canada; stateProvince: Ontario; county: Ottawa; locality: Ottawa city garden; verbatimLatitude: 45.356; verbatimLongitude: -75.707; verbatimEventDate: 10-Aug-2007; lifeStage: Adult; catalogNumber: CAM0114; recordedBy: H. Goulet; otherCatalogNumbers: CNCHZ304-09; identifiedBy: Jose Fernandez-Triana; institutionID: Canadian National Collection of Insects, Arachnids and Nematodes 
bk. country: Canada; stateProvince: Ontario; county: Heron Bay; municipality: Pukaskwa National Park; verbatimElevation: 631; verbatimLatitude: 48.601; verbatimLongitude: -86.289; verbatimEventDate: 01-Sep-2013; lifeStage: Adult; catalogNumber: BIOUG14107-D02; recordedBy: Cavan Harpur; otherCatalogNumbers: CNPKO1368-14; identifiedBy: Angela Telfer; institutionID: Biodiversity Institute of Ontario

bl. country: Canada; stateProvince: Ontario; county: Almonte; locality: $5 \mathrm{~km} \mathrm{NW}$ of Almonte, Hwy 49, Burnt Land, Alvar Prov. Park; verbatimLatitude: 45.255; verbatimLongitude: -76.140; verbatimEventDate: 29-May-2008; lifeStage: Adult; catalogNumber: CAM0345; recordedBy: Goulet \& Fernandez; otherCatalogNumbers: $\underline{\mathrm{CNCHZ535-09}}$; identifiedBy: Jose Fernandez-Triana; institutionID: Canadian National Collection of Insects, Arachnids and Nematodes

bm. $\quad$ country: Canada; stateProvince: Newfoundland and Labrador; county: St-David`s; locality: St-David’s, fallow field; verbatimLatitude: 48.205; verbatimLongitude: -58.866; verbatimEventDate: 10-Jul-2008; lifeStage: Adult; catalogNumber: CAM0963; recordedBy: H. Goulet, A. Badiss, C. Boudeault; otherCatalogNumbers: CNCHX093-09; identifiedBy: Jose Fernandez-Triana; institutionID: Canadian National Collection of Insects, Arachnids and Nematodes

bn. country: Canada; stateProvince: Quebec; county: Belle-Anse; locality: Belle-Anse; verbatimLatitude: 48.625; verbatimLongitude: -64.178; verbatimEventDate: 26-Jul-2008; lifeStage: Adult; catalogNumber: CAM0288; recordedBy: H. Goulet, A. Badiss, C. Boudeault; otherCatalog Numbers: CNCHZ478-09; identifiedBy: Jose Fernandez-Triana; institutionID: Canadian National Collection of Insects, Arachnids and Nematodes

bo. country: Canada; stateProvince: Ontario; county: Almonte; locality: $5 \mathrm{~km} \mathrm{NW}$ of Almonte, Hwy 49, Burnt Land, Alvar Prov. Park; verbatimLatitude: 45.255; verbatimLongitude: -76.140; verbatimEventDate: 29-May-2008; lifeStage: Adult; catalogNumber: CAM0341; recordedBy: Goulet \& Fernandez; otherCatalogNumbers: CNCHZ531-09; identifiedBy: Jose Fernandez-Triana; institutionID: Canadian National Collection of Insects, Arachnids and Nematodes

bp. country: Canada; stateProvince: Ontario; county: Ottawa; locality: Ottawa city garden; verbatimLatitude: 45.356; verbatimLongitude: -75.707; verbatimEventDate: 08-Sep-2007; lifeStage: Adult; catalogNumber: CAM0106; recordedBy: H. Goulet; otherCatalogNumbers: CNCHZ296-09; identifiedBy: Jose Fernandez-Triana; institutionID: Canadian National Collection of Insects, Arachnids and Nematodes

bq. $\quad$ country: Canada; stateProvince: Ontario; county: Thousand Islands NP; municipality: Jones Creek by Mallorytown, County Rd. 5; verbatimElevation: 117; verbatimLatitude: 44.475; verbatimLongitude: -75.865; verbatimEventDate: 12-Aug-2012; lifeStage: Adult; catalogNumber: BIOUG06823-E06; recordedBy: Thousand Islands NP; otherCatalogNumbers: CNSLS115-13; institutionID: Biodiversity Institute of Ontario

br. $\quad$ country: Canada; stateProvince: Manitoba; locality: Gardenton; verbatimElevation: 292; verbatimLatitude: 49.079; verbatimLongitude: -96.766; verbatimEventDate: 11-Jun-2007; lifeStage: Adult; catalogNumber: HYM00001019; recordedBy: Goulet \& Boudreault; otherCatalogNumbers: ASWA305-08; identifiedBy: Jose Fernandez-Triana; institutionID: Canadian National Collection of Insects, Arachnids and Nematodes

bs. country: Canada; stateProvince: Ontario; county: Ottawa; locality: Ottawa city garden; verbatimLatitude: 45.356; verbatimLongitude: -75.707; verbatimEventDate: 01-Sep-2007; lifeStage: Adult; catalogNumber: CAM0154; recordedBy: H. Goulet;

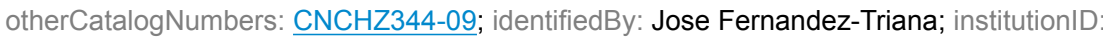
Canadian National Collection of Insects, Arachnids and Nematodes

bt. country: Canada; stateProvince: Ontario; county: Almonte; locality: 5 km NW of Almonte, Hwy 49, Burnt Land, Alvar Prov. Park; verbatimLatitude: 45.255; verbatimLongitude: 
-76.140; verbatimEventDate: 29-May-2008; lifeStage: Adult; catalogNumber: CAM0340; recordedBy: Goulet \& Fernandez; otherCatalogNumbers: CNCHZ530-09; identifiedBy: Jose Fernandez-Triana; institutionID: Canadian National Collection of Insects, Arachnids and Nematodes

bu. $\quad$ country: Canada; stateProvince: Manitoba; locality: Vita Tallgrass prairie; verbatimLatitude: 49.147; verbatimLongitude: -96.681; verbatimEventDate: 11-Jun-2007; lifeStage: Adult; catalogNumber: HYM00001028; recordedBy: Goulet \& Boudreault; otherCatalogNumbers: ASWA314-08; identifiedBy: Jose Fernandez-Triana; institutionID: Canadian National Collection of Insects, Arachnids and Nematodes

bv. $\quad$ country: Canada; stateProvince: Manitoba; locality: Gardenton; verbatimElevation: 292; verbatimLatitude: 49.079; verbatimLongitude: -96.766; verbatimEventDate: 11-Jun-2007; lifeStage: Adult; catalogNumber: HYM00001024; recordedBy: Goulet \& Boudreault; otherCatalogNumbers: ASWA310-08; identifiedBy: Jose Fernandez-Triana; institutionID: Canadian National Collection of Insects, Arachnids and Nematodes

bw. country: Canada; stateProvince: Ontario; county: Ottawa; locality: Ottawa city garden; verbatimLatitude: 45.356; verbatimLongitude: -75.707; verbatimEventDate: 01-Sep-2007; lifeStage: Adult; catalogNumber: CAM0161; recordedBy: H. Goulet;

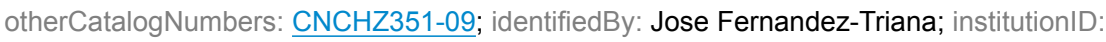
Canadian National Collection of Insects, Arachnids and Nematodes

bx. $\quad$ country: Canada; stateProvince: Quebec; county: Mingan Archipelago National Park Reserve; municipality: Quarry Island; locality: bog near forest edge; verbatimLatitude: 50.214; verbatimLongitude: -63.798; verbatimEventDate: 06-Aug-2013; lifeStage: Adult; catalogNumber: BIOUG12486-D10; recordedBy: Park Staff; otherCatalogNumbers: CNMIG236-14; identifiedBy: Angela Telfer; institutionID: Biodiversity Institute of Ontario

by. $\quad$ country: Canada; stateProvince: Ontario; county: Point Pelee NP; municipality: 15km SE of Leamington; locality: Cactus Patch Meadow off Bike Trail on Route to Visitors Centre; verbatimElevation: 174; verbatimLatitude: 41.934; verbatimLongitude: -82.514; verbatimEventDate: 23-Jun-2010; lifeStage: Adult; catalogNumber: PCPP10-0466; recordedBy: T.F.Mitterboeck, C.Vandermeer, V.Junea, C.Sobel; otherCatalogNumbers: BBHYN072-10; identifiedBy: Julie K. Stahlhut; institutionID: Biodiversity Institute of Ontario

bz. country: Canada; stateProvince: Nova Scotia; county: Kejimkujik National Park; locality: Jeremy`s Bay Campground, near Ampitheater off of Campfire Circle; verbatimElevation: 116; verbatimLatitude: 44.407; verbatimLongitude: -65.245; verbatimEventDate: 18Jul-2013; lifeStage: Adult; catalogNumber: BIOUG12736-B05; recordedBy: J.Garber, M.Davis; otherCatalogNumbers: CNKJN622-14; identifiedBy: Angela Telfer; institutionID: Biodiversity Institute of Ontario

ca. $\quad$ country: United States; stateProvince: Florida; county: Kissimmee Prairie Preserve SP; locality: mesic hammock; verbatimElevation: 15 ; verbatimLatitude: 27.582 ; verbatimLongitude: -81.048; verbatimEventDate: 07-Mar-2010; lifeStage: Adult; catalogNumber: 10BBHYM-1225; recordedBy: Biobus 2010; otherCatalogNumbers: BBHYH202-10; identifiedBy: Jose Fernandez-Triana; institutionID: Biodiversity Institute of Ontario

cb. $\quad$ country: Canada; stateProvince: Manitoba; municipality: Gardenton; verbatimElevation: 292; verbatimLatitude: 49.079; verbatimLongitude: -96.766; verbatimEventDate: 11Jun-2007; lifeStage: Adult; catalogNumber: HYM00001004; recordedBy: Goulet \& Boudreault; otherCatalogNumbers: ASWA290-08; identifiedBy: Jose Fernandez-Triana; institutionID: Canadian National Collection of Insects, Arachnids and Nematodes

cc. country: Canada; stateProvince: Ontario; county: Ottawa; locality: Ottawa city garden; verbatimLatitude: 45.356; verbatimLongitude: -75.707; verbatimEventDate: 19-Sep-2007; 
lifeStage: Adult; catalogNumber: CAM0008; recordedBy: H. Goulet; otherCatalogNumbers: CNCHZ198-09; identifiedBy: Jose Fernandez-Triana; institutionID: Canadian National Collection of Insects, Arachnids and Nematodes

cd. $\quad$ country: Canada; stateProvince: Ontario; county: Wellington County; municipality: Guelph; locality: Biodiversity Institute of Ontario; verbatimElevation: 335; verbatimLatitude: 43.528; verbatimLongitude: -80.229; verbatimEventDate: 27-Jun-2013; lifeStage: Adult; catalogNumber: BIOUG08799-G08; recordedBy: BIO Collections Staff; otherCatalogNumbers: MBIOE1410-13; institutionID: Biodiversity Institute of Ontario

ce. country: Canada; stateProvince: Ontario; county: Ottawa; locality: Mixed forest near Manotick; verbatimLatitude: 45.235; verbatimLongitude: -75.624; lifeStage: Adult; catalogNumber: CAM0521; recordedBy: A. Bennett; otherCatalogNumbers: CNCHZ1141-09; identifiedBy: Jose Fernandez-Triana; institutionID: Canadian National Collection of Insects, Arachnids and Nematodes

cf. country: Canada; stateProvince: Ontario; county: Thousand Islands NP; municipality: Jones Creek by Mallorytown, County Rd. 5; verbatimElevation: 117; verbatimLatitude: 44.475; verbatimLongitude: -75.865; verbatimEventDate: 15-Sep-2012; lifeStage: Adult; catalogNumber: BIOUG04041-A02; recordedBy: Thousand Islands NP; otherCatalogNumbers: CNSLK104-12; institutionID: Biodiversity Institute of Ontario

cg. $\quad$ country: Canada; stateProvince: Ontario; county: Thousand Islands NP; municipality: Jones Creek by Mallorytown, County Rd. 5; verbatimElevation: 117; verbatimLatitude: 44.475; verbatimLongitude: -75.865; verbatimEventDate: 21-Aug-2012; lifeStage: Adult; catalogNumber: BIOUG03979-A07; recordedBy: Thousand Islands NP; otherCatalogNumbers: CNSLI393-12; institutionID: Biodiversity Institute of Ontario

ch. country: Canada; stateProvince: Ontario; county: Thousand Islands NP; municipality: Jones Creek by Mallorytown, County Rd. 5; verbatimElevation: 117; verbatimLatitude: 44.475; verbatimLongitude: -75.865; verbatimEventDate: 21-Aug-2012; lifeStage: Adult; catalogNumber: BIOUG03979-B04; recordedBy: Thousand Islands NP; otherCatalogNumbers: CNSLI402-12; institutionID: Biodiversity Institute of Ontario

ci. country: Canada; stateProvince: Ontario; county: Haldimond-Dunn Townline; municipality: Windy Bluff; verbatimElevation: 179; verbatimLatitude: 42.861 ; verbatimLongitude: -79.703; verbatimEventDate: 01-Aug-2010; lifeStage: Adult; catalogNumber: BIOUG00991-B03; recordedBy: Alex Smith; otherCatalogNumbers: ASGLF363-11; identifiedBy: Jose Fernandez-Triana; institutionID: Research Collection of M. Alex Smith

cj. country: Canada; stateProvince: Ontario; county: Ottawa; locality: Ottawa city garden; verbatimLatitude: 45.356; verbatimLongitude: -75.707; verbatimEventDate: 01-Sep-2007; lifeStage: Adult; catalogNumber: CAM0166; recordedBy: H. Goulet;

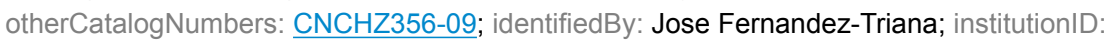
Canadian National Collection of Insects, Arachnids and Nematodes

ck. country: Canada; stateProvince: Ontario; county: Ottawa; locality: Ottawa city garden; verbatimLatitude: 45.356; verbatimLongitude: -75.707; verbatimEventDate: 08-Sep-2007; lifeStage: Adult; catalogNumber: CAM0103; recordedBy: H. Goulet; otherCatalogNumbers: CNCHZ293-09; identifiedBy: Jose Fernandez-Triana; institutionID: Canadian National Collection of Insects, Arachnids and Nematodes

cl. country: Canada; stateProvince: Ontario; county: Rouge National Urban Park; locality:

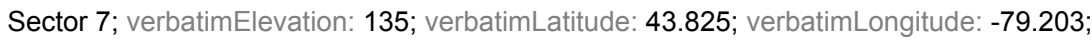
verbatimEventDate: 15-Sep-2013; lifeStage: Adult; catalogNumber: BIOUG07898-C04; recordedBy: BIO Team; otherCatalogNumbers: RBINA1006-13; institutionID: Biodiversity Institute of Ontario

cm. country: Canada; stateProvince: Ontario; county: Wellington County; municipality: Guelph; locality: Biodiversity Institute of Ontario; verbatimElevation: 335; 
verbatimLatitude: 43.528; verbatimLongitude: -80.229; verbatimEventDate: 04-Oct-2013; lifeStage: Adult; catalogNumber: BIOUG09931-A07; recordedBy: BIO Collections Staff; otherCatalogNumbers: MBION389-14; institutionID: Biodiversity Institute of Ontario

cn. country: Canada; stateProvince: Quebec; county: La Mauricie National Park; municipality: Saint-Mathieu west park entrance near Visitor Centre; locality: primarily conifer forest stand (balsam fir, Epicea glauca, birch); verbatimElevation: 219; verbatimLatitude: 46.651; verbatimLongitude: -72.970; verbatimEventDate: 01-Aug-2013; lifeStage: Adult; catalogNumber: BIOUG12977-D11; recordedBy: Medo Toure; otherCatalogNumbers: CNLMG1235-14; identifiedBy: Angela Telfer; institutionID: Biodiversity Institute of Ontario co. $\quad$ country: Canada; stateProvince: Ontario; county: Guelph; municipality: John F. Ross Collegiate \& Vocational Institute; locality: EQP-CLL-524; verbatimElevation: 329; verbatimLatitude: 43.562; verbatimLongitude: -80.247; verbatimEventDate: 27-Sep-2013; lifeStage: Adult; catalogNumber: BIOUG08641-G07; recordedBy: M.Neerhof, S.Lipskie; otherCatalogNumbers: SMTPD3273-13; institutionID: Biodiversity Institute of Ontario

cp. $\quad$ country: United States; stateProvince: North Carolina; county: Swain County; locality: Smokemont; verbatimEventDate: 07-Jun-1976; lifeStage: Adult; catalogNumber:

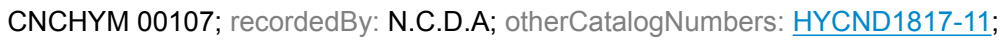
identifiedBy: Jose Fernandez-Triana; institutionID: Canadian National Collection of Insects, Arachnids and Nematodes

cq. $\quad$ country: Canada; stateProvince: Newfoundland and Labrador; county: St-Andrew`s; locality: St-Andrew`s, fallow field; verbatimLatitude: 47.793; verbatimLongitude: -59.233; verbatimEventDate: 18-Jul-2008; lifeStage: Adult; catalogNumber: CAM0938; recordedBy: H. Goulet, A. Badiss, C. Boudeault; otherCatalogNumbers: $\mathbf{C N C H X 0 6 8 - 0 9 ;}$ identifiedBy: Jose Fernandez-Triana; institutionID: Canadian National Collection of Insects, Arachnids and Nematodes

cr. country: Canada; stateProvince: Ontario; county: Almonte; locality: 5 km NW of Almonte, Hwy 49, Burnt Land, Alvar Prov. Park; verbatimLatitude: 45.255; verbatimLongitude: -76.140; verbatimEventDate: 29-May-2008; lifeStage: Adult; catalogNumber: CAM0348; recordedBy: Goulet \& Fernandez; otherCatalogNumbers: CNCHZ538-09; identifiedBy: Jose Fernandez-Triana; institutionID: Canadian National Collection of Insects, Arachnids and Nematodes

cs. $\quad$ country: Canada; stateProvince: Ontario; county: St. Marys; municipality: Little Falls Public School; locality: EQP-CLL-554; verbatimElevation: 305; verbatimLatitude: 43.259; verbatimLongitude: -81.142; verbatimEventDate: 27-Sep-2013; lifeStage: Adult; catalogNumber: BIOUG08647-G07; recordedBy: Lisa Cairncross; otherCatalogNumbers: SMTPD3831-13; institutionID: Biodiversity Institute of Ontario

ct. country: Canada; stateProvince: Ontario; county: Thousand Islands NP; municipality: Jones Creek by Mallorytown, County Rd. 5; verbatimElevation: 117; verbatimLatitude: 44.475; verbatimLongitude: -75.865; verbatimEventDate: 21-Aug-2012; lifeStage: Adult; catalogNumber: BIOUG03979-B03; recordedBy: Thousand Islands NP; otherCatalogNumbers: CNSLI401-12; institutionID: Biodiversity Institute of Ontario

cu. country: Canada; stateProvince: Ontario; county: Almonte; locality: 5 km NW of Almonte, Hwy 49, Burnt Land, Alvar Prov. Park; verbatimLatitude: 45.255; verbatimLongitude: -76.140; verbatimEventDate: 29-May-2008; lifeStage: Adult; catalogNumber: CAM0346; recordedBy: Goulet \& Fernandez; otherCatalogNumbers: CNCHZ536-09; identifiedBy: Jose Fernandez-Triana; institutionID: Canadian National Collection of Insects, Arachnids and Nematodes

cv. $\quad$ country: Canada; stateProvince: Ontario; locality: Scheck Nature Reserve; verbatimLatitude: 44.349; verbatimLongitude: -76.895; verbatimEventDate: 05-Oct-2011; lifeStage: Adult; catalogNumber: BIOUG01795-D07; recordedBy: J. Cossey; 
otherCatalogNumbers: NCCC726-11; identifiedBy: BOLD ID Engine; institutionID: Biodiversity Institute of Ontario

cw. $\quad$ country: Canada; stateProvince: Ontario; county: Heron Bay; municipality: Pukaskwa National Park; verbatimElevation: 631; verbatimLatitude: 48.601; verbatimLongitude: -86.289; verbatimEventDate: 09-Sep-2013; lifeStage: Adult; catalogNumber:

BIOUG11996-F03; recordedBy: Cavan Harpur; otherCatalogNumbers: CNPKK1009-14; institutionID: Biodiversity Institute of Ontario

cx. country: Canada; stateProvince: Nova Scotia; county: Kejimkujik National Park; locality: Jeremy`s Bay Campground, near Ampitheater off of Campfire Circle; verbatimElevation: 116; verbatimLatitude: 44.407; verbatimLongitude: -65.245; verbatimEventDate: 11Jul-2013; lifeStage: Adult; catalogNumber: BIOUG11597-E04; recordedBy: D.Crossland, K.Rowter; otherCatalogNumbers: CNKJE1961-14; institutionID: Biodiversity Institute of Ontario

cy. $\quad$ country: Canada; stateProvince: Ontario; county: Leeds; locality: Leeds-Granville Co., forest; verbatimLatitude: 44.629; verbatimLongitude: -76.359; verbatimEventDate: 01Oct-2008; lifeStage: Adult; catalogNumber: CAM0445; recordedBy: S. Peck;

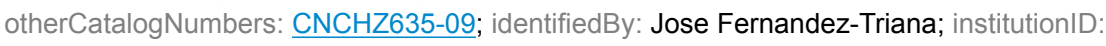
Canadian National Collection of Insects, Arachnids and Nematodes

cz. $\quad$ country: Canada; stateProvince: Ontario; county: Rouge National Urban Park; locality: Sector 8; verbatimElevation: 167; verbatimLatitude: 43.859; verbatimLongitude: -79.209; verbatimEventDate: 15-Sep-2013; lifeStage: Adult; catalogNumber: BIOUG07903-F12; recordedBy: BIO Team; otherCatalogNumbers: RBINA3743-13; institutionID: Biodiversity Institute of Ontario

da. country: Canada; stateProvince: Ontario; county: Ottawa; locality: Mixed forest near Manotick; verbatimLatitude: 45.235; verbatimLongitude: -75.624; lifeStage: Adult; catalogNumber: CAM0527; recordedBy: A. Bennett; otherCatalogNumbers: CNCHZ1147-09; identifiedBy: Jose Fernandez-Triana; institutionID: Canadian National Collection of Insects, Arachnids and Nematodes

db. country: Canada; stateProvince: Ontario; municipality: Carden Alvar; locality: North Bear; verbatimLatitude: 44.695; verbatimLongitude: -79.039; verbatimEventDate: 06-Oct-2011; lifeStage: Adult; catalogNumber: BIOUG01657-C11; recordedBy: J. Cossey; otherCatalogNumbers: NCCC130-11; institutionID: Biodiversity Institute of Ontario

dc. $\quad$ country: Canada; stateProvince: New Brunswick; county: Kouchibouguac NP; municipality: Research House; verbatimElevation: 52; verbatimLatitude: 46.771; verbatimLongitude: -65.006; verbatimEventDate: 20-Aug-2009; lifeStage: Adult; catalogNumber: 09BBEHY-2030; recordedBy: BIObus 2009; otherCatalogNumbers: BBHYF031-10; identifiedBy: Jose Fernandez-Triana; institutionID: Biodiversity Institute of Ontario

dd. country: Canada; stateProvince: Ontario; county: Toronto; municipality: Rouge National Urban Park, Trap 1; verbatimElevation: 136; verbatimLatitude: 43.822; verbatimLongitude: -79.190; verbatimEventDate: 14-Sep-2013; lifeStage: Adult; catalogNumber: BIOUG09770-A04; recordedBy: Lydia Attard, Kyla Greenham; otherCatalogNumbers: CNROT251-13; institutionID: Biodiversity Institute of Ontario

de. country: Canada; stateProvince: Ontario; county: Ottawa; locality: Mixed forest near Manotick; verbatimLatitude: 45.235; verbatimLongitude: -75.624; lifeStage: Adult; catalogNumber: CAM0531; recordedBy: A. Bennett; otherCatalogNumbers: CNCHZ1151-09; identifiedBy: Jose Fernandez-Triana; institutionID: Canadian National Collection of Insects, Arachnids and Nematodes

df. country: Canada; stateProvince: Ontario; county: Ottawa; locality: Ottawa city garden; verbatimLatitude: 45.356; verbatimLongitude: -75.707; verbatimEventDate: 19-Sep-2007; 
lifeStage: Adult; catalogNumber: CAM0007; recordedBy: H. Goulet; otherCatalogNumbers: CNCHZ197-09; identifiedBy: Jose Fernandez-Triana; institutionID: Canadian National Collection of Insects, Arachnids and Nematodes

dg. country: Canada; stateProvince: Quebec; county: Mingan Archipelago National Park Reserve; municipality: Quarry Island; locality: bog near forest edge; verbatimLatitude: 50.214; verbatimLongitude: -63.798; verbatimEventDate: 06-Aug-2013; lifeStage: Adult; catalogNumber: BIOUG12487-D03; recordedBy: Park Staff; otherCatalogNumbers: CNMIG324-14; identifiedBy: Angela Telfer; institutionID: Biodiversity Institute of Ontario

dh. country: Canada; stateProvince: Ontario; locality: Brighton; verbatimEventDate: 24Jul-1956; sex: Female; lifeStage: Adult; catalogNumber: MIC 000086;

otherCatalogNumbers: MHABR086-09; identifiedBy: Jose Fernandez-Triana; institutionID: Canadian National Collection of Insects, Arachnids and Nematodes

di. country: Canada; stateProvince: Ontario; county: Guelph; municipality: 25 Division St.; verbatimElevation: 326; verbatimLatitude: 43.554; verbatimLongitude: -80.264; verbatimEventDate: 01-Nov-2009; lifeStage: Adult; catalogNumber: ASGLE-0880; recordedBy: Alex Smith; otherCatalogNumbers: ASGLE690-10; identifiedBy: Jose Fernandez-Triana; institutionID: Research Collection of M. Alex Smith

dj. country: Canada; stateProvince: Ontario; county: Thousand Islands NP; municipality: Jones Creek by Mallorytown, County Rd. 5; verbatimElevation: 117; verbatimLatitude: 44.475; verbatimLongitude: -75.865; verbatimEventDate: 02-Sep-2012; lifeStage: Adult; catalogNumber: BIOUG03887-F12; recordedBy: Thousand Islands NP; otherCatalogNumbers: CNSLJ045-12; institutionID: Biodiversity Institute of Ontario

dk. country: Canada; stateProvince: Ontario; county: Paris; municipality: Holy Family School; locality: EQP-CLL-560; verbatimElevation: 250; verbatimLatitude: 43.207; verbatimLongitude: -80.391; verbatimEventDate: 27-Sep-2013; lifeStage: Adult; catalogNumber: BIOUG08665-D12; recordedBy: Anne Parkhill; otherCatalogNumbers: SMTPD4966-13; institutionID: Biodiversity Institute of Ontario

dl. country: Canada; stateProvince: Ontario; county: Wellington County; municipality: Guelph; locality: Biodiversity Institute of Ontario; verbatimElevation: 335; verbatimLatitude: 43.528; verbatimLongitude: -80.229; verbatimEventDate: 22-Aug-2013; lifeStage: Adult; catalogNumber: BIOUG09515-E11; recordedBy: BIO Collections Staff; otherCatalogNumbers: MBIOI1222-13; institutionID: Biodiversity Institute of Ontario

dm. country: Canada; stateProvince: Ontario; county: Ottawa; locality: Ottawa city garden; verbatimLatitude: 45.356; verbatimLongitude: -75.707; verbatimEventDate: 13-Jul-2007; lifeStage: Adult; catalogNumber: CAM0022; recordedBy: H. Goulet;

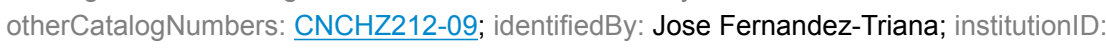
Canadian National Collection of Insects, Arachnids and Nematodes

dn. country: Canada; stateProvince: Ontario; county: Leeds; locality: Leeds-Granville Co., forest; verbatimLatitude: 44.629; verbatimLongitude: -76.359; verbatimEventDate: 01 Oct-2008; lifeStage: Adult; catalogNumber: CAM0446; recordedBy: S. Peck; otherCatalogNumbers: CNCHZ636-09; identifiedBy: Jose Fernandez-Triana; institutionID: Canadian National Collection of Insects, Arachnids and Nematodes

do. country: Canada; stateProvince: Ontario; county: Leeds and Grenville; municipality: Elizabethtown-Kitley; locality: 4452 Rowsome Rd., Elizabethtown; verbatimElevation:

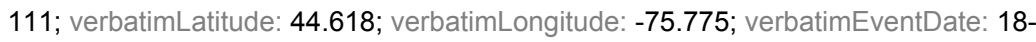
Aug-2011; lifeStage: Adult; catalogNumber: BIOUG01631-A09; recordedBy: James Sones; otherCatalogNumbers: JSAUG1440-11; identifiedBy: BOLD ID Engine; institutionID: Biodiversity Institute of Ontario

dp. country: Canada; stateProvince: Ontario; county: Cochrane; locality: Cochrane \& Kapuscaning Forest Reserves; verbatimLatitude: 49.398; verbatimLongitude: -80.472; 
verbatimEventDate: 12-Aug-2007; lifeStage: Adult; catalogNumber: CAM0635; recordedBy: N. Islam; otherCatalogNumbers: ASCNC020-09; identifiedBy: Jose Fernandez-Triana; institutionID: Canadian National Collection of Insects, Arachnids and Nematodes

dq. country: Canada; stateProvince: Ontario; county: Almonte; locality: $5 \mathrm{~km} \mathrm{NW}$ of Almonte, Hwy 49, Burnt Land, Alvar Prov. Park; verbatimLatitude: 45.255; verbatimLongitude: -76.140; verbatimEventDate: 29-May-2008; lifeStage: Adult; catalogNumber: CAM0335; recordedBy: Goulet \& Fernandez; otherCatalogNumbers: CNCHZ525-09; identifiedBy: Jose Fernandez-Triana; institutionID: Canadian National Collection of Insects, Arachnids and Nematodes

dr. country: Canada; stateProvince: Nova Scotia; locality: Kejimkujik National Park, Grafton Lake; verbatimLatitude: 44.383; verbatimLongitude: -65.202; verbatimEventDate: 04Aug-2009; lifeStage: Adult; catalogNumber: GOU 0570; recordedBy: J. Fernandez; otherCatalogNumbers: WOMIA340-11; identifiedBy: Jose Fernandez-Triana; institutionID: Canadian National Collection of Insects, Arachnids and Nematodes

ds. $\quad$ country: Canada; stateProvince: Ontario; county: Rouge National Urban Park; locality: Sector 8; verbatimElevation: 167; verbatimLatitude: 43.859; verbatimLongitude: -79.209; verbatimEventDate: 15-Sep-2013; lifeStage: Adult; catalogNumber: BIOUG07902-D08; recordedBy: BIO Team; otherCatalogNumbers: RBINA3620-13; institutionID: Biodiversity Institute of Ontario

dt. country: Canada; stateProvince: Manitoba; locality: Gardenton; verbatimElevation: 292; verbatimLatitude: 49.079; verbatimLongitude: -96.766; verbatimEventDate: 11-Jun-2007; lifeStage: Adult; catalogNumber: HYM00001026; recordedBy: Goulet \& Boudreault; otherCatalogNumbers: ASWA312-08; identifiedBy: Jose Fernandez-Triana; institutionID: Canadian National Collection of Insects, Arachnids and Nematodes

du. country: Canada; stateProvince: Ontario; county: Ottawa; locality: Mixed forest near Manotick; verbatimLatitude: 45.235; verbatimLongitude: -75.624; lifeStage: Adult; catalogNumber: CAM0523; recordedBy: A. Bennett; otherCatalogNumbers: CNCHZ1143-09; identifiedBy: Jose Fernandez-Triana; institutionID: Canadian National Collection of Insects, Arachnids and Nematodes

dv. country: Canada; stateProvince: Ontario; county: Almonte; locality: $5 \mathrm{~km}$ NW of Almonte, Hwy 49, Burnt Land, Alvar Prov. Park; verbatimLatitude: 45.255; verbatimLongitude: -76.140; verbatimEventDate: 29-May-2008; lifeStage: Adult; catalogNumber: CAM0356;

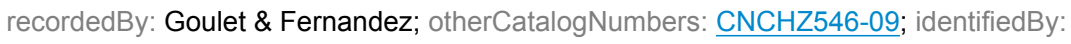
Jose Fernandez-Triana; institutionID: Canadian National Collection of Insects, Arachnids and Nematodes

dw. country: United States; stateProvince: Alaska; locality: Anchorage; verbatimElevation: 33; verbatimLatitude: 61.166; verbatimLongitude: -149.766; verbatimEventDate: 08Jun-2003; lifeStage: Adult; catalogNumber: $\mathrm{CNCH} 1358$; recordedBy: Goulet \& Boudreault; otherCatalogNumbers: CNCHW328-09; identifiedBy: Jose Fernandez-Triana; institutionID: Canadian National Collection of Insects, Arachnids and Nematodes

dx. country: Canada; stateProvince: Ontario; county: Ottawa; locality: Blackburn; verbatimEventDate: 09-Jun-1939; sex: Male; lifeStage: Adult; catalogNumber: MIC 000083; recordedBy: O. Peck; otherCatalogNumbers: MHABR083-09; identifiedBy: Jose Fernandez-Triana; institutionID: Canadian National Collection of Insects, Arachnids and Nematodes

dy. $\quad$ country: Canada; stateProvince: Quebec; county: La Mauricie National Park; municipality: Saint-Mathieu west park entrance near Visitor Centre; locality: primarily conifer forest stand (balsam fir, Epicea glauca, birch); verbatimElevation: 219; verbatimLatitude: 46.651; verbatimLongitude: -72.970; verbatimEventDate: 01-Aug-2013; lifeStage: Adult; 
catalogNumber: BIOUG12977-D01; recordedBy: Medo Toure; otherCatalogNumbers: CNLMG1225-14; identifiedBy: Angela Telfer; institutionID: Biodiversity Institute of Ontario dz. country: Canada; stateProvince: Ontario; county: Georgian Bay Islands National Park; municipality: Beausoleil Island, Cedar Spring Campground; locality: Mixed forest; verbatimElevation: 177; verbatimLatitude: 44.852; verbatimLongitude: -79.874; verbatimEventDate: 19-Aug-2013; lifeStage: Adult; catalogNumber: BIOUG11331-F04; recordedBy: J. Howard, C. Johnstone; otherCatalogNumbers: CNGBN1394-14; identifiedBy: Angela Telfer; institutionID: Biodiversity Institute of Ontario

ea. country: Canada; stateProvince: Ontario; county: Thousand Islands NP; municipality: Jones Creek by Mallorytown, County Rd. 5; verbatimElevation: 117; verbatimLatitude: 44.475; verbatimLongitude: -75.865; verbatimEventDate: 21-Aug-2012; lifeStage: Adult; catalogNumber: BIOUG03863-G08; recordedBy: Thousand Islands NP; otherCatalogNumbers: CNSLI089-12; institutionID: Biodiversity Institute of Ontario

eb. $\quad$ country: Canada; stateProvince: Ontario; county: Toronto; municipality: Rouge National Urban Park, Trap 1; verbatimElevation: 136; verbatimLatitude: 43.822; verbatimLongitude: -79.190; verbatimEventDate: 27-Aug-2013; lifeStage: Adult; catalogNumber: BIOUG09765-B03; recordedBy: Lydia Attard, Kyla Greenham; otherCatalogNumbers: CNROS484-13; institutionID: Biodiversity Institute of Ontario

ec. country: Canada; stateProvince: Ontario; county: Manitoulin Island; locality: Little Current, prairie alvar; verbatimLatitude: 45.975; verbatimLongitude: -81.904; verbatimEventDate: 04-Jun-2008; lifeStage: Adult; catalogNumber: CAM0410; recordedBy: Goulet, Boudreault \& Fernandez; otherCatalogNumbers: CNCHZ600-09; identifiedBy: Jose Fernandez-Triana; institutionID: Canadian National Collection of Insects, Arachnids and Nematodes

ed. country: Canada; stateProvince: Ontario; county: Ottawa; locality: Ottawa city garden; verbatimLatitude: 45.356; verbatimLongitude: -75.707; verbatimEventDate: 10-Aug-2007; lifeStage: Adult; catalogNumber: CAM0113; recordedBy: H. Goulet;

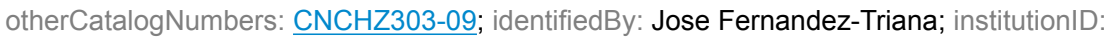
Canadian National Collection of Insects, Arachnids and Nematodes

ee. $\quad$ country: Canada; stateProvince: Ontario; locality: London; verbatimLatitude: 43.030; verbatimLongitude: -81.206; verbatimEventDate: 07-Jul-2003; lifeStage: Adult; catalogNumber: WMIC 0517; recordedBy: L. Gualtieri; otherCatalogNumbers: CNCHX802-09; identifiedBy: Jose Fernandez-Triana; institutionID: Canadian National Collection of Insects, Arachnids and Nematodes

ef. country: Canada; stateProvince: Ontario; county: Heron Bay; municipality: Pukaskwa National Park; verbatimElevation: 631; verbatimLatitude: 48.601; verbatimLongitude: -86.289; verbatimEventDate: 09-Sep-2013; lifeStage: Adult; catalogNumber: BIOUG11999-F03; recordedBy: Cavan Harpur; otherCatalogNumbers: CNPKK1294-14; institutionID: Biodiversity Institute of Ontario

eg. country: Canada; stateProvince: Manitoba; county: Churchill; municipality: $23 \mathrm{~km} \mathrm{SE}$ Churchill, Twin Lakes Road fen; locality: fen grasses and willows, $5 \mathrm{~km}$ down Twin lakes rd; verbatimLatitude: 58.672; verbatimLongitude: -93.839; verbatimEventDate: 29Jul-2010; lifeStage: Adult; catalogNumber: BIOUG00726-B07; recordedBy: Brandon Laforest; otherCatalogNumbers: JBHCl247-11; identifiedBy: Jose Fernandez-Triana; institutionID: Biodiversity Institute of Ontario

eh. country: Canada; stateProvince: Ontario; county: Ottawa; locality: Ottawa city garden; verbatimLatitude: 45.356; verbatimLongitude: -75.707; verbatimEventDate: 01-Sep-2007; lifeStage: Adult; catalogNumber: CAM0151; recordedBy: H. Goulet; otherCatalogNumbers: CNCHZ341-09; identifiedBy: Jose Fernandez-Triana; institutionID: Canadian National Collection of Insects, Arachnids and Nematodes 
ei. country: Canada; stateProvince: Ontario; county: Ottawa; locality: Mixed forest near Manotick; verbatimLatitude: 45.235; verbatimLongitude: -75.624; lifeStage: Adult; catalogNumber: CAM0524; recordedBy: A. Bennett; otherCatalogNumbers:

CNCHZ1144-09; identifiedBy: Jose Fernandez-Triana; institutionID: Canadian National Collection of Insects, Arachnids and Nematodes

ej. $\quad$ country: Canada; stateProvince: Ontario; county: Thousand Islands NP; municipality: Jones Creek by Mallorytown, County Rd. 5; verbatimElevation: 117; verbatimLatitude: 44.475; verbatimLongitude: -75.865; verbatimEventDate: 24-Jun-2012; lifeStage: Adult; catalogNumber: BIOUG03123-B05; recordedBy: Thousand Islands NP; otherCatalogNumbers: CNSLE365-12; institutionID: Biodiversity Institute of Ontario

ek. country: Canada; stateProvince: Ontario; county: Ottawa; locality: Ottawa city garden; verbatimLatitude: 45.356; verbatimLongitude: -75.707; verbatimEventDate: 30-May-2007; lifeStage: Adult; catalogNumber: CAM0013; recordedBy: H. Goulet; otherCatalogNumbers: CNCHZ203-09; identifiedBy: Jose Fernandez-Triana; institutionID: Canadian National Collection of Insects, Arachnids and Nematodes

el. $\quad$ country: Canada; stateProvince: Ontario; county: Ottawa; locality: Britannia; verbatimEventDate: 20-Jun-1947; sex: Male; lifeStage: Adult; catalogNumber: MIC 000082; recordedBy: G. Shewell; otherCatalogNumbers: MHABR082-09; identifiedBy: Jose Fernandez-Triana; institutionID: Canadian National Collection of Insects, Arachnids and Nematodes

em. $\quad$ country: Canada; stateProvince: Ontario; county: Heron Bay; municipality: Pukaskwa National Park; verbatimElevation: 631; verbatimLatitude: 48.601; verbatimLongitude: -86.289; verbatimEventDate: 01-Sep-2013; lifeStage: Adult; catalogNumber: BIOUG14171-B01; recordedBy: Cavan Harpur; otherCatalogNumbers: CNPKO2958-14; identifiedBy: Angela Telfer; institutionID: Biodiversity Institute of Ontario

en. $\quad$ country: Canada; stateProvince: Newfoundland and Labrador; county: St-Andrew`s; locality: St-Andrew`s, fallow field; verbatimLatitude: 47.793; verbatimLongitude: -59.233; verbatimEventDate: 18-Jul-2008; lifeStage: Adult; catalogNumber: CAM0942;

recordedBy: H. Goulet, A. Badiss, C. Boudeault; otherCatalogNumbers: CNCHX072-09; identifiedBy: Jose Fernandez-Triana; institutionID: Canadian National Collection of Insects, Arachnids and Nematodes

eo. $\quad$ country: Canada; stateProvince: Ontario; county: Wellington County; municipality: Guelph; locality: Biodiversity Institute of Ontario; verbatimElevation: 335; verbatimLatitude: 43.528; verbatimLongitude: -80.229; verbatimEventDate: 19-Sep-2013; lifeStage: Adult; catalogNumber: BIOUG09803-F06; recordedBy: BIO Collections Staff; otherCatalogNumbers: MBIOK076-14; institutionID: Biodiversity Institute of Ontario

ep. $\quad$ country: Canada; stateProvince: Ontario; county: Thousand Islands NP; municipality: Jones Creek by Mallorytown, County Rd. 5; verbatimElevation: 117; verbatimLatitude: 44.475; verbatimLongitude: -75.865; verbatimEventDate: 15-Jul-2012; lifeStage: Adult; catalogNumber: BIOUG07004-C04; recordedBy: Thousand Islands NP;

otherCatalogNumbers: CNSLQ251-13; institutionID: Biodiversity Institute of Ontario

eq. country: Canada; stateProvince: Ontario; county: Ottawa; locality: Mixed forest near Manotick; verbatimLatitude: 45.235; verbatimLongitude: -75.624; lifeStage: Adult; catalogNumber: CAM0529; recordedBy: A. Bennett; otherCatalogNumbers: CNCHZ1149-09; identifiedBy: Jose Fernandez-Triana; institutionID: Canadian National Collection of Insects, Arachnids and Nematodes

er. $\quad$ country: Canada; stateProvince: Ontario; county: Ottawa; locality: Ottawa city garden; verbatimLatitude: 45.356; verbatimLongitude: -75.707; verbatimEventDate: 26-Jun-2007; lifeStage: Adult; catalogNumber: CAM0074; recordedBy: H. Goulet; 
otherCatalogNumbers: CNCHZ264-09; identifiedBy: Jose Fernandez-Triana; institutionID: Canadian National Collection of Insects, Arachnids and Nematodes

es. $\quad$ country: Canada; stateProvince: Ontario; county: Thousand Islands NP; municipality: Jones Creek by Mallorytown, County Rd. 5; verbatimElevation: 117; verbatimLatitude: 44.475; verbatimLongitude: -75.865; verbatimEventDate: 12-Aug-2012; lifeStage: Adult; catalogNumber: BIOUG06823-E08; recordedBy: Thousand Islands NP; otherCatalogNumbers: CNSLS117-13; institutionID: Biodiversity Institute of Ontario

et. $\quad$ country: Canada; stateProvince: Ontario; county: Paris; municipality: Holy Family School; locality: EQP-CLL-560; verbatimElevation: 250; verbatimLatitude: 43.207 ; verbatimLongitude: -80.391; verbatimEventDate: 27-Sep-2013; lifeStage: Adult; catalogNumber: BIOUG08665-A11; recordedBy: Anne Parkhill; otherCatalogNumbers: SMTPD4929-13; institutionID: Biodiversity Institute of Ontario

eu. $\quad$ country: Canada; stateProvince: Ontario; county: Leeds and Grenville; municipality: Elizabethtown-Kitley; locality: 4452 Rowsome Rd., Elizabethtown; verbatimElevation: 111; verbatimLatitude: 44.618 ; verbatimLongitude: -75.775 ; verbatimEventDate: 14 Sep-2011; lifeStage: Adult; catalogNumber: BIOUG01631-G11; recordedBy: James Sones; otherCatalogNumbers: JSSEP758-11; identifiedBy: BOLD ID Engine; institutionID: Biodiversity Institute of Ontario

ev. country: Canada; stateProvince: Quebec; county: La Mauricie National Park; municipality: Saint-Mathieu west park entrance near Visitor Centre; locality: primarily conifer forest stand (balsam fir, Epicea glauca, birch); verbatimElevation: 219; verbatimLatitude: 46.651; verbatimLongitude: -72.970; verbatimEventDate: 01-Aug-2013; lifeStage: Adult; catalogNumber: BIOUG12974-A03; recordedBy: Medo Toure; otherCatalogNumbers: CNLMG906-14; identifiedBy: Angela Telfer; institutionID: Biodiversity Institute of Ontario

ew. country: Canada; stateProvince: Ontario; county: Leeds; locality: Leeds-Granville Co., forest; verbatimLatitude: 44.629; verbatimLongitude: -76.359; verbatimEventDate: 01 Oct-2008; lifeStage: Adult; catalogNumber: CAM0447; recordedBy: S. Peck; otherCatalogNumbers: $\mathrm{CNCHZ637-09;}$; identifiedBy: Jose Fernandez-Triana; institutionID: Canadian National Collection of Insects, Arachnids and Nematodes

ex. country: Canada; stateProvince: Ontario; county: Leeds and Grenville; municipality: Elizabethtown-Kitley; locality: 4452 Rowsome Rd., Elizabethtown; verbatimElevation: 111; verbatimLatitude: 44.618 ; verbatimLongitude: -75.775 ; verbatimEventDate: 18 Aug-2011; lifeStage: Adult; catalogNumber: BIOUG01597-F04; recordedBy: James Sones; otherCatalogNumbers: JSAUG449-11; identifiedBy: BOLD ID Engine; institutionID: Biodiversity Institute of Ontario

ey. $\quad$ country: Canada; stateProvince: Ontario; county: St. Marys; municipality: Little Falls Public School; locality: EQP-CLL-554; verbatimElevation: 305; verbatimLatitude: 43.259; verbatimLongitude: -81.142; verbatimEventDate: 27-Sep-2013; lifeStage: Adult; catalogNumber: BIOUG08647-D01; recordedBy: Lisa Cairncross; otherCatalogNumbers: SMTPD3789-13; institutionID: Biodiversity Institute of Ontario

ez. country: Canada; stateProvince: Ontario; municipality: Carden Alvar; locality: North Bear; verbatimLatitude: 44.695; verbatimLongitude: -79.039; verbatimEventDate: 06-Oct-2011; lifeStage: Adult; catalogNumber: BIOUG01657-F02; recordedBy: J. Cossey; otherCatalogNumbers: NCCC157-11; institutionID: Biodiversity Institute of Ontario

fa. country: Canada; stateProvince: Ontario; county: Toronto; municipality: Rouge National Urban Park, Trap 1; verbatimElevation: 136; verbatimLatitude: 43.822; verbatimLongitude: -79.190; verbatimEventDate: 27-Aug-2013; lifeStage: Adult; catalogNumber: BIOUG09765-A12; recordedBy: Lydia Attard, Kyla Greenham; otherCatalogNumbers: CNROS481-13; institutionID: Biodiversity Institute of Ontario 
fb. $\quad$ country: Canada; stateProvince: Ontario; county: Thousand Islands NP; municipality: Jones Creek by Mallorytown, County Rd. 5; verbatimElevation: 117; verbatimLatitude: 44.475; verbatimLongitude: -75.865; verbatimEventDate: 02-Sep-2012; lifeStage: Adult; catalogNumber: BIOUG03979-H08; recordedBy: Thousand Islands NP; otherCatalogNumbers: CNSLJ189-12; institutionID: Biodiversity Institute of Ontario

fc. country: Canada; stateProvince: Ontario; county: Almonte; locality: $5 \mathrm{~km} \mathrm{NW}$ of Almonte, Hwy 49, Burnt Land, Alvar Prov. Park; verbatimLatitude: 45.255; verbatimLongitude: -76.140; verbatimEventDate: 29-May-2008; lifeStage: Adult; catalogNumber: CAM0350; recordedBy: Goulet \& Fernandez; otherCatalogNumbers: CNCHZ540-09; identifiedBy: Jose Fernandez-Triana; institutionID: Canadian National Collection of Insects, Arachnids and Nematodes

fd. country: Canada; stateProvince: Ontario; county: Point Pelee NP; municipality: Cactus Field; verbatimElevation: 168; verbatimLatitude: 41.939; verbatimLongitude: -82.516; verbatimEventDate: 07-Sep-2012; lifeStage: Adult; catalogNumber: BIOUG03931-F01; recordedBy: H.Brown; otherCatalogNumbers: CNPPJ1314-12; institutionID: Biodiversity Institute of Ontario

fe. country: Canada; stateProvince: Ontario; county: Wellington County; municipality: Guelph; locality: Biodiversity Institute of Ontario; verbatimElevation: 335; verbatimLatitude: 43.528; verbatimLongitude: -80.229; verbatimEventDate: 04-Oct-2013; lifeStage: Adult; catalogNumber: BIOUG09930-G10; recordedBy: BIO Collections Staff; otherCatalogNumbers: MBION369-14; institutionID: Biodiversity Institute of Ontario

ff. country: Canada; stateProvince: Ontario; county: St. Marys; municipality: Little Falls Public School; locality: EQP-CLL-554; verbatimElevation: 305; verbatimLatitude: 43.259; verbatimLongitude: -81.142; verbatimEventDate: 27-Sep-2013; lifeStage: Adult; catalogNumber: BIOUG08648-H10; recordedBy: Lisa Cairncross; otherCatalogNumbers: SMTPD3941-13; institutionID: Biodiversity Institute of Ontario

fg. Country: Canada; stateProvince: Ontario; county: Toronto; municipality: Rouge National Urban Park, Trap 1; verbatimElevation: 136; verbatimLatitude: 43.822; verbatimLongitude: -79.190; verbatimEventDate: 03-Sep-2013; lifeStage: Adult; catalogNumber: BIOUG08752-A08; recordedBy: Lydia Attard, Kyla Greenham; otherCatalogNumbers: CNROJ908-13; institutionID: Biodiversity Institute of Ontario

fh. $\quad$ country: Canada; stateProvince: Quebec; county: Mingan Archipelago National Park Reserve; municipality: Quarry Island; locality: bog near forest edge; verbatimLatitude: 50.214; verbatimLongitude: -63.798; verbatimEventDate: 06-Aug-2013; lifeStage: Adult; catalogNumber: BIOUG12497-F12; recordedBy: Park Staff; otherCatalogNumbers: CNMIG1286-14; identifiedBy: Angela Telfer; institutionID: Biodiversity Institute of Ontario

fi. country: Canada; stateProvince: Ontario; county: Paris; municipality: Holy Family School; locality: EQP-CLL-560; verbatimElevation: 250; verbatimLatitude: 43.207; verbatimLongitude: -80.391; verbatimEventDate: 27-Sep-2013; lifeStage: Adult; catalogNumber: BIOUG08665-F02; recordedBy: Anne Parkhill; otherCatalogNumbers: SMTPD4980-13; institutionID: Biodiversity Institute of Ontario

fj. country: Canada; stateProvince: Ontario; county: Wellington County; municipality: Guelph; locality: Biodiversity Institute of Ontario; verbatimElevation: 335; verbatimLatitude: 43.528; verbatimLongitude: -80.229; verbatimEventDate: 04-Oct-2013; lifeStage: Adult; catalogNumber: BIOUG09930-E06; recordedBy: BIO Collections Staff; otherCatalogNumbers: MBION341-14; institutionID: Biodiversity Institute of Ontario

fk. country: Canada; stateProvince: Ontario; county: Ottawa; locality: Ottawa city garden; verbatimLatitude: 45.356; verbatimLongitude: -75.707; verbatimEventDate: 30-Jul-2007; lifeStage: Adult; catalogNumber: CAM0120; recordedBy: H. Goulet; 
otherCatalogNumbers: $\mathrm{CNCHZ310-09;}$; identifiedBy: Jose Fernandez-Triana; institutionID: Canadian National Collection of Insects, Arachnids and Nematodes

fl. country: Canada; stateProvince: Ontario; municipality: Carden Alvar; locality: North Bear; verbatimLatitude: 44.695; verbatimLongitude: -79.039; verbatimEventDate: 06-Oct-2011; lifeStage: Adult; catalogNumber: BIOUG01661-E11; recordedBy: J. Cossey; otherCatalogNumbers: NCCC249-11; institutionID: Biodiversity Institute of Ontario

fm. country: Canada; stateProvince: Ontario; county: Toronto; municipality: Rouge National Urban Park, Trap 1; verbatimElevation: 136; verbatimLatitude: 43.822; verbatimLongitude: -79.190; verbatimEventDate: 27-Aug-2013; lifeStage: Adult; catalogNumber: BIOUG09765-A06; recordedBy: Lydia Attard, Kyla Greenham; otherCatalogNumbers: CNROS475-13; institutionID: Biodiversity Institute of Ontario

fn. country: Canada; stateProvince: Ontario; county: Manitoulin Island; locality: Misery Bay Prov. Park; verbatimElevation: 185 ; verbatimLatitude: 45.792 ; verbatimLongitude: -82.750; verbatimEventDate: 03-Jun-2008; lifeStage: Adult; catalogNumber: CAM0389; recordedBy: Boudreault, Goulet \& Fernandez; otherCatalogNumbers: $\mathrm{CNCHZ579-09}$; identifiedBy: Jose Fernandez-Triana; institutionID: Canadian National Collection of Insects, Arachnids and Nematodes

fo. $\quad$ country: Canada; stateProvince: Manitoba; locality: Gardenton; verbatimElevation: 292; verbatimLatitude: 49.079; verbatimLongitude: -96.766; verbatimEventDate: 11-Jun-2007; lifeStage: Adult; catalogNumber: HYM00001020; recordedBy: Goulet \& Boudreault; otherCatalogNumbers: ASWA306-08; identifiedBy: Jose Fernandez-Triana; institutionID: Canadian National Collection of Insects, Arachnids and Nematodes

fp. $\quad$ country: Canada; stateProvince: Quebec; county: Belle-Anse; locality: Belle-Anse; verbatimLatitude: 48.625; verbatimLongitude: -64.178; verbatimEventDate: 26-Jul-2008; lifeStage: Adult; catalogNumber: CAM0286; recordedBy: H. Goulet, A. Badiss, C.

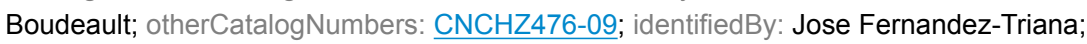
institutionID: Canadian National Collection of Insects, Arachnids and Nematodes

fq. country: Canada; stateProvince: Ontario; county: Ottawa; locality: Ottawa city garden; verbatimLatitude: 45.356; verbatimLongitude: -75.707; verbatimEventDate: 01-Sep-2007; lifeStage: Adult; catalogNumber: CAM0163; recordedBy: H. Goulet; otherCatalogNumbers: CNCHZ353-09; identifiedBy: Jose Fernandez-Triana; institutionID: Canadian National Collection of Insects, Arachnids and Nematodes

fr. country: Canada; stateProvince: Ontario; county: Almonte; locality: $5 \mathrm{~km}$ NW of Almonte, Hwy 49, Burnt Land, Alvar Prov. Park; verbatimLatitude: 45.255; verbatimLongitude: -76.140; verbatimEventDate: 29-May-2008; lifeStage: Adult; catalogNumber: CAM0351; recordedBy: Goulet \& Fernandez; otherCatalogNumbers: CNCHZ541-09; identifiedBy: Jose Fernandez-Triana; institutionID: Canadian National Collection of Insects, Arachnids and Nematodes

fs. $\quad$ country: United States; stateProvince: Massachusetts; county: Barnstable Country; municipality: Woods Hole|Woods Hole Village; locality: Urban parkland: Meadow/ Anthropogenic; verbatimLatitude: 41.529; verbatimLongitude: -70.670; verbatimEventDate: 29-Sep-2010; lifeStage: Adult; catalogNumber: TDWG-0384; recordedBy: BIObus: A.Borisenko, R.Labbee, V.Levesque-Beaudin, M.Milton, J.Smith, S.Ratnasingham; otherCatalogNumbers: TDWGB248-10; institutionID: Biodiversity Institute of Ontario

ft. country: Canada; stateProvince: Ontario; county: Manitoulin Island; locality: Misery Bay Prov. Park; verbatimElevation: 185; verbatimLatitude: 45.792; verbatimLongitude: -82.750; verbatimEventDate: 03-Jun-2008; lifeStage: Adult; catalogNumber: CAM0413; recordedBy: Boudreault, Goulet \& Fernandez; otherCatalogNumbers: CNCHZ603-09; 
identifiedBy: Jose Fernandez-Triana; institutionID: Canadian National Collection of Insects, Arachnids and Nematodes

fu. country: Canada; stateProvince: Ontario; county: Thousand Islands NP; municipality: Jones Creek by Mallorytown, County Rd. 5; verbatimElevation: 117; verbatimLatitude: 44.475; verbatimLongitude: -75.865; verbatimEventDate: 12-Aug-2012; lifeStage: Adult; catalogNumber: BIOUG06823-D07; recordedBy: Thousand Islands NP; otherCatalogNumbers: CNSLS104-13; institutionID: Biodiversity Institute of Ontario

fv. country: United States; stateProvince: Georgia; locality: Forsyth; verbatimEventDate: 08Oct-1970; lifeStage: Adult; catalogNumber: CNCHYM 00106; recordedBy: F. Naumann; otherCatalogNumbers: HYCND1816-11; identifiedBy: Jose Fernandez-Triana; institutionID: Canadian National Collection of Insects, Arachnids and Nematodes

fw. country: Canada; stateProvince: Ontario; county: Teeswater; municipality: Sacred Heart School; locality: EQP-CLL-568; verbatimElevation: 314; verbatimLatitude: 43.992; verbatimLongitude: -81.282; verbatimEventDate: 27-Sep-2013; lifeStage: Adult; catalogNumber: BIOUG08499-C11; recordedBy: Kristen McGuire; otherCatalogNumbers: SMTPD1460-13; institutionID: Biodiversity Institute of Ontario

fx. country: Canada; stateProvince: Ontario; county: Almonte; locality: 5 km NW of Almonte, Hwy 49, Burnt Land, Alvar Prov. Park; verbatimLatitude: 45.255; verbatimLongitude: -76.140; verbatimEventDate: 29-May-2008; lifeStage: Adult; catalogNumber: CAM0360; recordedBy: Goulet \& Fernandez; otherCatalogNumbers: CNCHZ550-09; identifiedBy: Jose Fernandez-Triana; institutionID: Canadian National Collection of Insects, Arachnids and Nematodes

fy. country: Canada; stateProvince: Ontario; county: Almonte; locality: $5 \mathrm{~km} \mathrm{NW}$ of Almonte, Hwy 49, Burnt Land, Alvar Prov. Park; verbatimLatitude: 45.255; verbatimLongitude: -76.140; verbatimEventDate: 29-May-2008; lifeStage: Adult; catalogNumber: CAM0349;

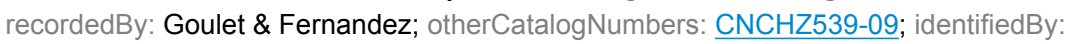
Jose Fernandez-Triana; institutionID: Canadian National Collection of Insects, Arachnids and Nematodes

fz. country: Canada; stateProvince: Ontario; county: Ottawa; locality: Ottawa city garden; verbatimLatitude: 45.356; verbatimLongitude: -75.707; verbatimEventDate: 10-Aug-2007; lifeStage: Adult; catalogNumber: CAM0115; recordedBy: H. Goulet; otherCatalogNumbers: CNCHZ305-09; identifiedBy: Jose Fernandez-Triana; institutionID: Canadian National Collection of Insects, Arachnids and Nematodes

ga. country: Canada; stateProvince: Ontario; locality: Grimsby; verbatimEventDate: 09Jun-1944; sex: Female; lifeStage: Adult; catalogNumber: MIC 000085; recordedBy: $\mathrm{H}$. Boyce; otherCatalogNumbers: MHABR085-09; identifiedBy: Jose Fernandez-Triana; institutionID: Canadian National Collection of Insects, Arachnids and Nematodes

gb. country: Canada; stateProvince: Ontario; county: Thousand Islands NP; municipality: Jones Creek by Mallorytown, County Rd. 5; verbatimElevation: 117; verbatimLatitude: 44.475; verbatimLongitude: -75.865; verbatimEventDate: 02-Sep-2012; lifeStage: Adult; catalogNumber: BIOUG03979-G06; recordedBy: Thousand Islands NP; otherCatalogNumbers: CNSLJ175-12; institutionID: Biodiversity Institute of Ontario

gc. country: Canada; stateProvince: Newfoundland and Labrador; county: St-David's; locality: St-David’s, fallow field; verbatimLatitude: 48.205; verbatimLongitude: -58.866 ; verbatimEventDate: 10-Jul-2008; lifeStage: Adult; catalogNumber: CAM0964; recordedBy: H. Goulet, A. Badiss, C. Boudeault; otherCatalogNumbers: CNCHX094-09; identifiedBy: Jose Fernandez-Triana; institutionID: Canadian National Collection of Insects, Arachnids and Nematodes

gd. country: Canada; stateProvince: Ontario; county: Ottawa; locality: Ottawa city garden; verbatimLatitude: 45.356; verbatimLongitude: -75.707; verbatimEventDate: 30-Jul-2007; 
lifeStage: Adult; catalogNumber: CAM0025; recordedBy: H. Goulet; otherCatalogNumbers: CNCHZ215-09; identifiedBy: Jose Fernandez-Triana; institutionID: Canadian National Collection of Insects, Arachnids and Nematodes

ge. country: Canada; stateProvince: Ontario; county: St. Lawrence Islands National Park; locality: Grenadier Island Center; verbatimEventDate: 21-Jul-1975; sex: Female; lifeStage: Adult; catalogNumber: MIC 000088; otherCatalogNumbers: MHABR088-09; identifiedBy: Jose Fernandez-Triana; institutionID: Canadian National Collection of Insects, Arachnids and Nematodes

gf. country: Canada; stateProvince: Ontario; county: Wellington County; municipality: Guelph; locality: Biodiversity Institute of Ontario; verbatimElevation: 335 ; verbatimLatitude: 43.528; verbatimLongitude: -80.229; verbatimEventDate: 27-Jun-2013; lifeStage: Adult; catalogNumber: BIOUG08807-A09; recordedBy: BIO Collections Staff; otherCatalogNumbers: MBIOE2286-13; institutionID: Biodiversity Institute of Ontario

gg. country: Canada; stateProvince: Ontario; county: Ottawa; locality: Ottawa city garden; verbatimLatitude: 45.356; verbatimLongitude: -75.707; verbatimEventDate: 08-Sep-2007; lifeStage: Adult; catalogNumber: CAM0101; recordedBy: H. Goulet;

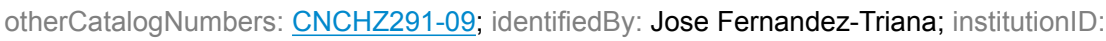
Canadian National Collection of Insects, Arachnids and Nematodes

gh. country: Canada; stateProvince: Ontario; county: Wellington County; municipality: Guelph; locality: Biodiversity Institute of Ontario; verbatimElevation: 335; verbatimLatitude: 43.528; verbatimLongitude: -80.229; verbatimEventDate: 27-Sep-2013; lifeStage: Adult; catalogNumber: BIOUG09925-F01; recordedBy: BIO Collections Staff; otherCatalogNumbers: MBIOL370-14; institutionID: Biodiversity Institute of Ontario

gi. country: Canada; stateProvince: Ontario; county: Wellington County; municipality: Guelph; locality: Biodiversity Institute of Ontario; verbatimElevation: 335; verbatimLatitude: 43.528; verbatimLongitude: -80.229; verbatimEventDate: 08-Aug-2013; lifeStage: Adult; catalogNumber: BIOUG09345-D07; recordedBy: BIO Collections Staff; otherCatalogNumbers: $\mathrm{MBIOH} 323-13$; institutionID: Biodiversity Institute of Ontario

gj. country: Canada; stateProvince: Ontario; county: Wellington County; municipality: Guelph; locality: Biodiversity Institute of Ontario; verbatimElevation: 335; verbatimLatitude: 43.528; verbatimLongitude: -80.229; verbatimEventDate: $27-S e p-2013$; lifeStage: Adult; catalogNumber: BIOUG09925-A01; recordedBy: BIO Collections Staff; otherCatalogNumbers: MBIOL310-14; institutionID: Biodiversity Institute of Ontario

gk. country: Canada; stateProvince: Quebec; county: La Mauricie National Park; municipality: Saint-Mathieu west park entrance near Visitor Centre; locality: primarily conifer forest stand (balsam fir, Epicea glauca, birch); verbatimElevation: 219; verbatimLatitude: 46.651; verbatimLongitude: -72.970; verbatimEventDate: 23-Jul-2013; lifeStage: Adult; catalogNumber: BIOUG14261-G11; recordedBy: Medo Toure; otherCatalogNumbers: CNLMP1391-14; identifiedBy: Angela Telfer; institutionID: Biodiversity Institute of Ontario gl. country: Canada; stateProvince: Ontario; county: Ottawa; locality: Ottawa city garden; verbatimLatitude: 45.356; verbatimLongitude: -75.707; verbatimEventDate: 26-Jun-2007; lifeStage: Adult; catalogNumber: CAM0062; recordedBy: H. Goulet; otherCatalogNumbers: CNCHZ252-09; identifiedBy: Jose Fernandez-Triana; institutionID: Canadian National Collection of Insects, Arachnids and Nematodes

gm. country: Canada; stateProvince: Ontario; county: Paris; municipality: Holy Family School; locality: EQP-CLL-560; verbatimElevation: 250; verbatimLatitude: 43.207; verbatimLongitude: -80.391; verbatimEventDate: 27-Sep-2013; lifeStage: Adult; catalogNumber: BIOUG08665-D10; recordedBy: Anne Parkhill; otherCatalogNumbers: SMTPD4964-13; institutionID: Biodiversity Institute of Ontario 
gn. country: Canada; stateProvince: Ontario; county: Ottawa; locality: Ottawa city garden; verbatimLatitude: 45.356; verbatimLongitude: -75.707; verbatimEventDate: 26-Jun-2007; lifeStage: Adult; catalogNumber: CAM0076; recordedBy: H. Goulet; otherCatalogNumbers: CNCHZ266-09; identifiedBy: Jose Fernandez-Triana; institutionID: Canadian National Collection of Insects, Arachnids and Nematodes

go. country: Canada; stateProvince: Ontario; county: Heron Bay; municipality: Pukaskwa National Park; verbatimElevation: 631; verbatimLatitude: 48.601; verbatimLongitude: -86.289; verbatimEventDate: 26-Aug-2013; lifeStage: Adult; catalogNumber:

BIOUG11771-G09; recordedBy: Cavan Harpur; otherCatalogNumbers: CNPKJ2482-14; institutionID: Biodiversity Institute of Ontario

gp. country: Canada; stateProvince: Ontario; county: Wellington County; municipality: Guelph; locality: Biodiversity Institute of Ontario; verbatimElevation: 335; verbatimLatitude: 43.528; verbatimLongitude: -80.229; verbatimEventDate: 19-Sep-2013; lifeStage: Adult; catalogNumber: BIOUG09807-D05; recordedBy: BIO Collections Staff; otherCatalogNumbers: MBIOK431-14; institutionID: Biodiversity Institute of Ontario

gq. country: Canada; stateProvince: Ontario; county: Ottawa; locality: Mixed forest near Manotick; verbatimLatitude: 45.235; verbatimLongitude: -75.624; lifeStage: Adult; catalogNumber: CAM0530; recordedBy: A. Bennett; otherCatalogNumbers:

CNCHZ1150-09; identifiedBy: Jose Fernandez-Triana; institutionID: Canadian National Collection of Insects, Arachnids and Nematodes

gr. Country: Canada; stateProvince: Manitoba; locality: Gardenton; verbatimElevation: 292; verbatimLatitude: 49.079; verbatimLongitude: -96.766; verbatimEventDate: 11-Jun-2007; lifeStage: Adult; catalogNumber: HYM00001021; recordedBy: Goulet \& Boudreault; otherCatalogNumbers: ASWA307-08; identifiedBy: Jose Fernandez-Triana; institutionID: Canadian National Collection of Insects, Arachnids and Nematodes

gs. country: Canada; stateProvince: Ontario; county: Ottawa; locality: Mixed forest near Manotick; verbatimLatitude: 45.235; verbatimLongitude: -75.624; lifeStage: Adult; catalogNumber: CAM0528; recordedBy: A. Bennett; otherCatalogNumbers: CNCHZ1148-09; identifiedBy: Jose Fernandez-Triana; institutionID: Canadian National Collection of Insects, Arachnids and Nematodes

gt. country: Canada; stateProvince: Saskatchewan; county: Prince Albert NP; municipality: Boundary Bog Trl.; verbatimElevation: 568; verbatimLatitude: 53.906; verbatimLongitude: -106.030; verbatimEventDate: 13-Aug-2010; lifeStage: Adult; catalogNumber: 10BBCHY-1304; recordedBy: BIObus 2010; otherCatalogNumbers: BBHYJ345-10; identifiedBy: Julie K. Stahlhut; institutionID: Biodiversity Institute of Ontario

gu. country: Canada; stateProvince: Ontario; county: Ottawa; locality: Ottwa city garden; verbatimLatitude: 45.356; verbatimLongitude: -75.707; verbatimEventDate: 06-Jul-2009; lifeStage: Adult; catalogNumber: CNCH1025; recordedBy: L. Masner; otherCatalogNumbers: CNCHX1040-09; identifiedBy: Jose Fernandez-Triana; institutionID: Canadian National Collection of Insects, Arachnids and Nematodes gv. country: Canada; stateProvince: Ontario; county: Manitoulin Island; locality: Misery Bay Prov. Park; verbatimElevation: 185; verbatimLatitude: 45.792; verbatimLongitude: -82.750; verbatimEventDate: 03-Jun-2008; lifeStage: Adult; catalogNumber: CAM0399; recordedBy: Boudreault, Goulet \& Fernandez; otherCatalogNumbers: CNCHZ589-09; identifiedBy: Jose Fernandez-Triana; institutionID: Canadian National Collection of Insects, Arachnids and Nematodes

gw. country: Canada; stateProvince: Ontario; county: Rouge National Urban Park; locality: Sector 8; verbatimElevation: 167; verbatimLatitude: 43.859; verbatimLongitude: -79.209; verbatimEventDate: 15-Sep-2013; lifeStage: Adult; catalogNumber: BIOUG07902-D06; 
recordedBy: BIO Team; otherCatalogNumbers: RBINA3618-13; institutionID: Biodiversity Institute of Ontario

gx. country: Canada; stateProvince: Ontario; county: St. Lawrence Islands National Park; locality: Thwartway Island; verbatimEventDate: 15-Aug-1976; sex: Female; lifeStage: Adult; catalogNumber: MIC 000089; otherCatalog Numbers: MHABR089-09; identifiedBy: Jose Fernandez-Triana; institutionID: Canadian National Collection of Insects, Arachnids and Nematodes

gy. country: Canada; stateProvince: Ontario; county: Leeds and Grenville; municipality: Elizabethtown-Kitley; locality: 4452 Rowsome Rd., Elizabethtown; verbatimElevation: 111; verbatimLatitude: 44.618; verbatimLongitude: -75.775 ; verbatimEventDate: 18 Aug-2011; lifeStage: Adult; catalogNumber: BIOUG01631-A05; recordedBy: James Sones; otherCatalogNumbers: JSAUG1436-11; identifiedBy: BOLD ID Engine; institutionID: Biodiversity Institute of Ontario

gz. $\quad$ country: Canada; stateProvince: Newfoundland and Labrador; county: Corner Brook; locality: Corner Brook, fallow field; verbatimLatitude: 48.956; verbatimLongitude: -57.911; verbatimEventDate: 17-Jul-2008; lifeStage: Adult; catalogNumber: CAM0941;

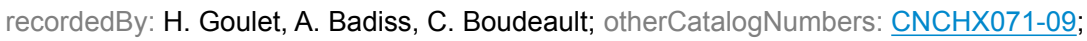
identifiedBy: Jose Fernandez-Triana; institutionID: Canadian National Collection of Insects, Arachnids and Nematodes

ha. country: Canada; stateProvince: Newfoundland and Labrador; county: St-David's; locality: St-David's, fallow field; verbatimLatitude: 48.205; verbatimLongitude: -58.866 ; verbatimEventDate: 10-Jul-2008; lifeStage: Adult; catalogNumber: CAM0961; recordedBy: H. Goulet, A. Badiss, C. Boudeault; otherCatalogNumbers: CNCHX091-09; identifiedBy: Jose Fernandez-Triana; institutionID: Canadian National Collection of Insects, Arachnids and Nematodes

hb. $\quad$ country: Canada; stateProvince: Ontario; county: Georgian Bay Islands National Park; municipality: Georgian Bay Islands National Park, Administration Office, 901 Wye Valley Rd.; locality: Marsh; verbatimElevation: 190; verbatimLatitude: 44.742; verbatimLongitude: -79.850; verbatimEventDate: 23-May-2013; lifeStage: Adult; catalogNumber: BIOUG10641-G12; recordedBy: Chris Johnstone; otherCatalogNumbers: CNGBJ1514-14; institutionID: Biodiversity Institute of Ontario

hc. country: Canada; stateProvince: Ontario; county: Wellington County; municipality: Guelph; locality: Biodiversity Institute of Ontario; verbatimElevation: 335; verbatimLatitude: 43.528; verbatimLongitude: -80.229; verbatimEventDate: 22-Aug-2013; lifeStage: Adult; catalogNumber: BIOUG09631-C02; recordedBy: BIO Collections Staff; otherCatalogNumbers: $\mathrm{MBIOI1284-13}$; institutionID: Biodiversity Institute of Ontario

hd. country: Canada; stateProvince: Ontario; county: Heron Bay; municipality: Pukaskwa National Park; verbatimElevation: 631; verbatimLatitude: 48.601; verbatimLongitude: -86.289; verbatimEventDate: 01-Sep-2013; lifeStage: Adult; catalogNumber: BIOUG14099-G10; recordedBy: Cavan Harpur; otherCatalogNumbers: CNPKO652-14; identifiedBy: Angela Telfer; institutionID: Biodiversity Institute of Ontario

he. $\quad$ country: Canada; stateProvince: Nova Scotia; county: Kejimkujik National Park; locality: Jeremy`s Bay Campground, near Ampitheater off of Campfire Circle; verbatimElevation: 116; verbatimLatitude: 44.407; verbatimLongitude: -65.245; verbatimEventDate: 25 Jul-2013; lifeStage: Adult; catalogNumber: BIOUG11758-F08; recordedBy: Matt Davis; otherCatalogNumbers: CNKJF1651-14; institutionID: Biodiversity Institute of Ontario hf. country: Canada; stateProvince: Saskatchewan; county: Prince Albert NP; municipality: Boundary Bog Trl.; verbatimElevation: 568; verbatimLatitude: 53.906; verbatimLongitude: -106.030; verbatimEventDate: 13-Aug-2010; lifeStage: Adult; catalogNumber: 
10BBCHY-1305; recordedBy: BIObus 2010; otherCatalogNumbers: BBHYJ346-10; identifiedBy: Julie K. Stahlhut; institutionID: Biodiversity Institute of Ontario

hg. country: Canada; stateProvince: Ontario; county: Ottawa; locality: Mixed forest near Manotick; verbatimLatitude: 45.235; verbatimLongitude: -75.624; lifeStage: Adult; catalogNumber: CAM0522; recordedBy: A. Bennett; otherCatalogNumbers:

CNCHZ1142-09; identifiedBy: Jose Fernandez-Triana; institutionID: Canadian National Collection of Insects, Arachnids and Nematodes

hh. country: Canada; stateProvince: Ontario; county: Almonte; locality: 5 km NW of Almonte, Hwy 49, Burnt Land, Alvar Prov. Park; verbatimLatitude: 45.255; verbatimLongitude: -76.140; verbatimEventDate: 29-May-2008; lifeStage: Adult; catalogNumber: CAM0347; recordedBy: Goulet \& Fernandez; otherCatalogNumbers: CNCHZ537-09; identifiedBy: Jose Fernandez-Triana; institutionID: Canadian National Collection of Insects, Arachnids and Nematodes

hi. $\quad$ country: United States; stateProvince: Louisiana; county: Evangeline County; locality: Bayou Chicot; verbatimEventDate: 23-Apr-1972; lifeStage: Adult; catalogNumber: CNCHYM 00105; recordedBy: D. Shanek; otherCatalogNumbers: HYCND1815-11; identifiedBy: Jose Fernandez-Triana; institutionID: Canadian National Collection of Insects, Arachnids and Nematodes

hj. country: Canada; stateProvince: Ontario; county: Heron Bay; municipality: Pukaskwa National Park; verbatimElevation: 631; verbatimLatitude: 48.601; verbatimLongitude: -86.289; verbatimEventDate: 01-Sep-2013; lifeStage: Adult; catalogNumber: BIOUG14170-E05; recordedBy: Cavan Harpur; otherCatalogNumbers: CNPKO2903-14; identifiedBy: Angela Telfer; institutionID: Biodiversity Institute of Ontario

hk. country: Canada; stateProvince: Ontario; county: Ottawa; locality: Ottawa city garden; verbatimLatitude: 45.356; verbatimLongitude: -75.707; verbatimEventDate: 23-Jul-2007; lifeStage: Adult; catalogNumber: CAM0054; recordedBy: H. Goulet;

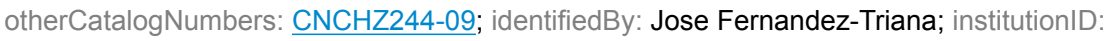
Canadian National Collection of Insects, Arachnids and Nematodes

hl. Country: Canada; stateProvince: Manitoba; county: Churchill; verbatimLatitude: 58.740; verbatimLongitude: -93.820; verbatimEventDate: 17-Aug-2006; lifeStage: Adult; catalogNumber: HYM00000665; otherCatalogNumbers: DSWAS984-07; identifiedBy: Jose Fernandez-Triana; institutionID: Canadian National Collection of Insects, Arachnids and Nematodes

hm. country: Canada; stateProvince: Ontario; county: Toronto; municipality: Rouge National Urban Park, Trap 1; verbatimElevation: 136; verbatimLatitude: 43.822; verbatimLongitude: -79.190; verbatimEventDate: 03-Sep-2013; lifeStage: Adult; catalogNumber: BIOUG08749-G01; recordedBy: Lydia Attard, Kyla Greenham; otherCatalogNumbers: CNROJ688-13; institutionID: Biodiversity Institute of Ontario

hn. country: United States; stateProvince: Massachusetts; county: Barnstable Country; municipality: Woods Hole|near memoriall circle cottage \#24; locality: Mixed deciduous; verbatimElevation: 16; verbatimLatitude: 41.530; verbatimLongitude: -70.655 ; verbatimEventDate: 29-Sep-2010; lifeStage: Adult; catalogNumber: TDWG-0167; recordedBy: BIObus: A.Borisenko, R.Labbee, V.Levesque-Beaudin, M.Milton, J.Smith, S.Ratnasingham; otherCatalogNumbers: TDWGB052-10; institutionID: Biodiversity Institute of Ontario

ho. $\quad$ country: Canada; stateProvince: Manitoba; county: Churchill; municipality: $23 \mathrm{~km} \mathrm{E}$ Churchill, Ramsay Creek; verbatimElevation: 10; verbatimLatitude: 58.731; verbatimLongitude: -93.780; verbatimEventDate: 23-Jul-2007; lifeStage: Adult; catalogNumber: 07PROBE-23393; recordedBy: A.Renaud; otherCatalogNumbers: 
ASWAX959-08; identifiedBy: Jose Fernandez-Triana; institutionID: Biodiversity Institute of Ontario

hp. country: Canada; stateProvince: Ontario; county: Rouge National Urban Park; locality: Sector 8; verbatimElevation: 167; verbatimLatitude: 43.859; verbatimLongitude: -79.209; verbatimEventDate: 15-Sep-2013; lifeStage: Adult; catalogNumber: BIOUG07902-D03; recordedBy: BIO Team; otherCatalogNumbers: RBINA3615-13; institutionID: Biodiversity Institute of Ontario

hq. country: Canada; stateProvince: Ontario; county: Ottawa; locality: Ottawa city garden; verbatimLatitude: 45.356; verbatimLongitude: -75.707; verbatimEventDate: 08-Sep-2007; lifeStage: Adult; catalogNumber: CAM0099; recordedBy: H. Goulet; otherCatalogNumbers: CNCHZ289-09; identifiedBy: Jose Fernandez-Triana; institutionID: Canadian National Collection of Insects, Arachnids and Nematodes

hr. country: Canada; stateProvince: Ontario; county: Perth East; municipality: Mornington Central School; locality: EQP-CLL-556; verbatimElevation: 380; verbatimLatitude: 43.614; verbatimLongitude: -80.885; verbatimEventDate: 27-Sep-2013; lifeStage: Adult; catalogNumber: BIOUG08565-E09; recordedBy: Barb Maschke; otherCatalogNumbers: SMTPD1793-13; institutionID: Biodiversity Institute of Ontario

hs. $\quad$ country: Canada; stateProvince: Ontario; county: Almonte; locality: 5 km NW of Almonte, Hwy 49, Burnt Land, Alvar Prov. Park; verbatimLatitude: 45.255; verbatimLongitude: -76.140; verbatimEventDate: 29-May-2008; lifeStage: Adult; catalogNumber: MIC 000666; recordedBy: Goulet \& Fernandez; otherCatalogNumbers: CNCHZ858-09; identifiedBy: Jose Fernandez-Triana; institutionID: Canadian National Collection of Insects, Arachnids and Nematodes

ht. country: Canada; stateProvince: Ontario; county: Ottawa; locality: Ottawa city garden; verbatimLatitude: 45.356; verbatimLongitude: -75.707; verbatimEventDate: 01-Sep-2007; lifeStage: Adult; catalogNumber: CAM0146; recordedBy: H. Goulet;

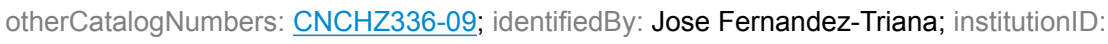
Canadian National Collection of Insects, Arachnids and Nematodes

hu. $\quad$ country: Canada; stateProvince: Ontario; county: Wellington County; municipality: Guelph; locality: Biodiversity Institute of Ontario; verbatimElevation: 335; verbatimLatitude: 43.528; verbatimLongitude: -80.229; verbatimEventDate: 05-Sep-2013; lifeStage: Adult; catalogNumber: BIOUG09801-C03; recordedBy: BIO Collections Staff; otherCatalogNumbers: MBIOJ817-13; institutionID: Biodiversity Institute of Ontario

hv. country: Canada; stateProvince: Prince Edward Island; locality: Blooming Point, fallow field; verbatimElevation: 6; verbatimLatitude: 46.408; verbatimLongitude: -62.951; verbatimEventDate: 23-Jul-2008; lifeStage: Adult; catalogNumber: MIC 000488; recordedBy: H. Goulet, A. Badiss, C. Boudeault; otherCatalogNumbers: $\mathrm{CNCHZ680-09}$; identifiedBy: Jose Fernandez-Triana; institutionID: Canadian National Collection of Insects, Arachnids and Nematodes

hw. country: Canada; stateProvince: Ontario; municipality: Carden Alvar; locality: Prairie Smoke; verbatimLatitude: 44.645; verbatimLongitude: -79.095 ; verbatimEventDate: 06 Oct-2011; lifeStage: Adult; catalogNumber: BIOUG01572-D01; recordedBy: J. Cossey; otherCatalog Numbers: NCCC037-11; institutionID: Biodiversity Institute of Ontario

hx. Country: Canada; stateProvince: Manitoba; locality: Gardenton; verbatimElevation: 292; verbatimLatitude: 49.079; verbatimLongitude: -96.766; verbatimEventDate: 11-Jun-2007; lifeStage: Adult; catalogNumber: HYM00001025; recordedBy: Goulet \& Boudreault; otherCatalogNumbers: ASWA311-08; identifiedBy: Jose Fernandez-Triana; institutionID: Canadian National Collection of Insects, Arachnids and Nematodes

hy. country: Canada; stateProvince: Ontario; county: Ottawa; locality: Ottawa city garden; verbatimLatitude: 45.356; verbatimLongitude: -75.707; verbatimEventDate: 26-Jun-2007; 
lifeStage: Adult; catalogNumber: CAM0072; recordedBy: H. Goulet; otherCatalogNumbers: CNCHZ262-09; identifiedBy: Jose Fernandez-Triana; institutionID: Canadian National Collection of Insects, Arachnids and Nematodes

hz. country: Canada; stateProvince: Ontario; county: Ottawa; locality: Ottawa city garden; verbatimLatitude: 45.356; verbatimLongitude: -75.707; verbatimEventDate: 30-Jul-2007; lifeStage: Adult; catalogNumber: CAM0024; recordedBy: H. Goulet;

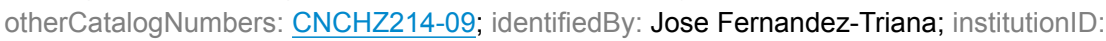
Canadian National Collection of Insects, Arachnids and Nematodes

ia. country: Canada; stateProvince: Ontario; county: Georgian Bay Islands National Park; municipality: Beausoleil Island, Cedar Spring Campground; locality: Mixed forest; verbatimElevation: 177; verbatimLatitude: 44.852; verbatimLongitude: -79.874; verbatimEventDate: 19-Aug-2013; lifeStage: Adult; catalogNumber: BIOUG11726-F01; recordedBy: J. Howard, C. Johnstone; otherCatalogNumbers: CNGBN2850-14; institutionID: Biodiversity Institute of Ontario

ib. $\quad$ country: Canada; stateProvince: Quebec; county: Mingan Archipelago National Park Reserve; municipality: Quarry Island; locality: bog near forest edge; verbatimLatitude: 50.214; verbatimLongitude: -63.798; verbatimEventDate: 06-Aug-2013; lifeStage: Adult; catalogNumber: BIOUG12460-D06; recordedBy: Park Staff; otherCatalogNumbers: CNMIG137-14; identifiedBy: Angela Telfer; institutionID: Biodiversity Institute of Ontario

ic. country: Canada; stateProvince: Ontario; county: Wellington County; municipality: Guelph; locality: Biodiversity Institute of Ontario; verbatimElevation: 335; verbatimLatitude: 43.528; verbatimLongitude: -80.229; verbatimEventDate: 04-Oct-2013; lifeStage: Adult; catalogNumber: BIOUG09930-G04; recordedBy: BIO Collections Staff; otherCatalogNumbers: MBION363-14; institutionID: Biodiversity Institute of Ontario

id. country: Canada; stateProvince: Ontario; county: Thousand Islands NP; municipality: Jones Creek by Mallorytown, County Rd. 5; verbatimElevation: 117; verbatimLatitude: 44.475; verbatimLongitude: -75.865; verbatimEventDate: 05-Aug-2012; lifeStage: Adult; catalogNumber: BIOUG03863-E06; recordedBy: Thousand Islands NP; otherCatalogNumbers: CNSLH124-12; institutionID: Biodiversity Institute of Ontario

ie. country: Canada; stateProvince: Ontario; county: Ottawa; locality: Ottawa city garden; verbatimLatitude: 45.356; verbatimLongitude: -75.707; verbatimEventDate: 10-Aug-2007; lifeStage: Adult; catalogNumber: CAM0039; recordedBy: H. Goulet; otherCatalogNumbers: CNCHZ229-09; identifiedBy: Jose Fernandez-Triana; institutionID: Canadian National Collection of Insects, Arachnids and Nematodes

if. country: Canada; stateProvince: Ontario; county: Manitoulin Island; locality: Little Current, prairie alvar; verbatimLatitude: 45.975; verbatimLongitude: -81.904; verbatimEventDate: 04-Jun-2008; lifeStage: Adult; catalogNumber: CAM0409; recordedBy: Goulet, Boudreault \& Fernandez; otherCatalogNumbers: $\mathrm{CNCHZ599-09;}$; identifiedBy: Jose Fernandez-Triana; institutionID: Canadian National Collection of Insects, Arachnids and Nematodes

Notes: This species is widely distributed in central and eastern North America. It was recorded from four provinces in Canada (MB, NS, ON, QC) by Fernandez-Triana (2010), but no locality records were provided at that time. Recently four more Canadian provinces and territories (AB, NB, NT, SK) and one state (AK) from the United States were added (Fernandez-Triana et al. 2014). Here we present additional information about the distribution of this species in the Nearctic, including two new provincial records for Canada (NL, PE) and one state (FL) from the United States, based on 
examined specimens from the $\mathrm{BIO}$ and $\mathrm{CNC}$ collections. Data for those specimens was extracted from BOLD.

The specimens of $A$. ensiger that rendered DNA barcodes comprise two BINS, BOLD:ACE6783 (ON, MB) and BOLD:AAA3764 (AB, ON, SK and some localities of southern US) (Suppl. material 4); whether they represent two different species or not will be studied in an upcoming paper.

\section{Apanteles sodalis (Haliday, 1834)}

\section{Materials}

a. country: Canada; stateProvince: British Columbia; locality: $11 \mathrm{~km} \mathrm{~S} \mathrm{of} \mathrm{Cawston;}$ verbatimElevation: 303; verbatimEventDate: 10-Jun-1998; lifeStage: Adult; catalogNumber: CBRA0492; recordedBy: J. Cossentine; otherCatalogNumbers: CNCHV584-10; identifiedBy: Jose Fernandez-Triana; institutionID: Canadian National Collection of Insects, Arachnids and Nematodes

b. $\quad$ country: Canada; stateProvince: Newfoundland and Labrador; locality: St. John's; verbatimEventDate: 05-Aug-2004; sex: Female; lifeStage: Adult; catalog Number: CBRA0493; recordedBy: H. Fry; otherCatalogNumbers: CNCHV585-10; identifiedBy: Jose Fernandez-Triana; institutionID: Canadian National Collection of Insects, Arachnids and Nematodes

c. country: Canada; stateProvince: British Columbia; locality: Chilliwack; verbatimEventDate: 27-Jun-1978; lifeStage: Adult; catalogNumber: CNCHYM 00215; recordedBy: Doganlar; otherCatalogNumbers: HYCNE025-11; identifiedBy: Jose Fernandez-Triana; institutionID: Canadian National Collection of Insects, Arachnids and Nematodes

d. country: Canada; stateProvince: British Columbia; locality: $11 \mathrm{~km} \mathrm{~S}$ of Cawston; verbatimElevation: 303; lifeStage: Adult; catalogNumber: CBRA0491; recordedBy: J. Cossentine; otherCatalogNumbers: CNCHV583-10; identifiedBy: Jose Fernandez-Triana; institutionID: Canadian National Collection of Insects, Arachnids and Nematodes

e. $\quad$ country: Canada; stateProvince: New Brunswick; locality: SE of Upsalq; verbatimEventDate: 19-Jul-1961; sex: Female; lifeStage: Adult; catalogNumber: MIC 000009; recordedBy: T. Renault; otherCatalogNumbers: MHABR009-09; identifiedBy: Jose Fernandez-Triana; institutionID: Canadian National Collection of Insects, Arachnids and Nematodes

f. country: Canada; stateProvince: British Columbia; locality: $11 \mathrm{~km} \mathrm{~S}$ of Cawston; verbatimElevation: 303; verbatimEventDate: 14-May-1998; lifeStage: Adult; catalogNumber: CBRA0490; recordedBy: J. Cossentine; otherCatalogNumbers: CNCHV582-10; identifiedBy: Jose Fernandez-Triana; institutionID: Canadian National Collection of Insects, Arachnids and Nematodes

g. country: Canada; stateProvince: British Columbia; locality: Burnaby; verbatimEventDate: 07-May-1977; sex: Female; lifeStage: Adult; catalogNumber: MIC 000007; recordedBy: F. Peters; otherCatalogNumbers: MHABR007-09; identifiedBy: Jose Fernandez-Triana; institutionID: Canadian National Collection of Insects, Arachnids and Nematodes

h. country: Canada; stateProvince: Newfoundland and Labrador; locality: St. John`s; verbatimEventDate: 05-Aug-2004; sex: Female; lifeStage: Adult; catalogNumber: MIC 000013; recordedBy: H. Fry; otherCatalogNumbers: MHABR013-09; identifiedBy: Jose Fernandez-Triana; institutionID: Canadian National Collection of Insects, Arachnids and Nematodes 
i. country: Canada; stateProvince: New Brunswick; locality: Green River Lab.; verbatimEventDate: 24-Jul-1961; sex: Female; lifeStage: Adult; catalogNumber: MIC 000010; recordedBy: T. Renault; otherCatalogNumbers: MHABR010-09; identifiedBy: Jose Fernandez-Triana; institutionID: Canadian National Collection of Insects, Arachnids and Nematodes

j. $\quad$ country: Canada; stateProvince: British Columbia; locality: 11 km S of Cawston; verbatimElevation: 303; verbatimEventDate: 14-May-1998; sex: Female; lifeStage: Adult; catalogNumber: MIC 000011; recordedBy: J. Cossentine; otherCatalogNumbers: MHABR011-09; identifiedBy: Jose Fernandez-Triana; institutionID: Canadian National Collection of Insects, Arachnids and Nematodes

k. $\quad$ country: Canada; stateProvince: Newfoundland and Labrador; locality: St. John`s; verbatimEventDate: 05-Aug-2004; sex: Male; lifeStage: Adult; catalogNumber: MIC 000014; recordedBy: H. Fry; otherCatalogNumbers: MHABR014-09; identifiedBy: Jose Fernandez-Triana; institutionID: Canadian National Collection of Insects, Arachnids and Nematodes

I. country: Canada; stateProvince: British Columbia; locality: Burnaby; verbatimEventDate: 07-May-1977; sex: Female; lifeStage: Adult; catalogNumber: MIC 000005; recordedBy: Docanlar; otherCatalogNumbers: MHABR005-09; identifiedBy: Jose Fernandez-Triana; institutionID: Canadian National Collection of Insects, Arachnids and Nematodes

m. country: Canada; stateProvince: Newfoundland and Labrador; locality: St. John`s; verbatimEventDate: 05-Aug-2004; sex: Female; lifeStage: Adult; catalogNumber: CBRA0495; recordedBy: H. Fry; otherCatalogNumbers: CNCHV587-10; identifiedBy: Jose Fernandez-Triana; institutionID: Canadian National Collection of Insects, Arachnids and Nematodes

n. country: Canada; stateProvince: Newfoundland and Labrador; locality: St. John`s; verbatimEventDate: 05-Aug-2004; sex: Female; lifeStage: Adult; catalogNumber: CBRA0494; recordedBy: H. Fry; otherCatalogNumbers: CNCHV586-10; identifiedBy: Jose Fernandez-Triana; institutionID: Canadian National Collection of Insects, Arachnids and Nematodes

o. country: Canada; stateProvince: British Columbia; locality: $11 \mathrm{~km} \mathrm{~S}$ of Cawston; verbatimElevation: 303; verbatimEventDate: 13-Jul-1998; sex: Male; lifeStage: Adult; catalogNumber: MIC 000012; recordedBy: J. Cossentine; otherCatalogNumbers: MHABR012-09; identifiedBy: Jose Fernandez-Triana; institutionID: Canadian National Collection of Insects, Arachnids and Nematodes

p. country: Canada; stateProvince: British Columbia; locality: Burnaby; verbatimEventDate: 07-May-1977; sex: Male; lifeStage: Adult; catalogNumber: MIC 000006; recordedBy: Doganlar; otherCatalogNumbers: MHABR006-09; identifiedBy: Jose Fernandez-Triana; institutionID: Canadian National Collection of Insects, Arachnids and Nematodes

q. $\quad$ country: Canada; stateProvince: British Columbia; locality: Summerland; verbatimEventDate: 02-Jun-1978; sex: Male; lifeStage: Adult; catalogNumber: MIC 000008; recordedBy: F. Peters; otherCatalogNumbers: MHABR008-09; identifiedBy: Jose Fernandez-Triana; institutionID: Canadian National Collection of Insects, Arachnids and Nematodes

Notes: This species is widely distributed in the Holarctic and it was recorded from three provinces in Canada (BC, NB, NL) by Fernandez-Triana (2010), but no locality records were provided at the time. Here we present new information about the distribution of this species in Canada, based on examined specimens from the $\mathrm{BIO}$ and $\mathrm{CNC}$ collections. Data for those specimens was extracted from BOLD. 
The specimens of $A$. sodalis that rendered DNA barcodes comprise two BINS: BOLD:AAM7223 (BC, NL) and BOLD:AAN1859 (BC) (Suppl. material 4); whether they represent two different species or not will be studied in an upcoming paper.

\section{Apanteles xanthostigma (Haliday, 1834)}

\section{Materials}

a. country: Canada; stateProvince: Newfoundland and Labrador; county: Gros Morne National Park; municipality: James Callaghan Trail (aka Gros Morne Trail); locality: mature conifer stand, balsam fir, wind damage; verbatimElevation: 39; verbatimLatitude: 49.569; verbatimLongitude: -57.830; verbatimEventDate: 17-Sep-2013; lifeStage: Adult; catalogNumber: BIOUG10637-H02; recordedBy: Anderson; otherCatalogNumbers: CNGMI700-14; institutionID: Biodiversity Institute of Ontario

b. $\quad$ country: Canada; stateProvince: Manitoba; county: Churchill; municipality: $16 \mathrm{~km} \mathrm{SW}$ Churchill, Churchill River Pump House; verbatimLatitude: 58.626; verbatimLongitude: -94.229; verbatimEventDate: 12-Jul-2007; lifeStage: Adult; catalogNumber: 07PROBE-22321; recordedBy: A.Renaud; otherCatalogNumbers: ASWAV862-08; identifiedBy: Jose Fernandez-Triana; institutionID: Biodiversity Institute of Ontario

c. country: Canada; stateProvince: Saskatchewan; county: Prince Albert NP; verbatimElevation: 627; verbatimLatitude: 53.850; verbatimLongitude: -106.078 ; verbatimEventDate: 10-Jul-2012; lifeStage: Adult; catalogNumber: BIOUG04486-G10; recordedBy: Rod Thompson, Eric Knight; otherCatalogNumbers: CNPAD006-13; identifiedBy: Jose Fernandez-Triana; institutionID: Biodiversity Institute of Ontario

d. country: Canada; stateProvince: British Columbia; county: Sorrento; locality: Sorrento, fallow field; verbatimLatitude: 50.779 ; verbatimLongitude: -119.502 ; verbatimEventDate: 28-May-2005; lifeStage: Adult; catalogNumber: MIC 000638; recordedBy: Goulet \& Boudreault; otherCatalogNumbers: CNCHZ830-09; identifiedBy: Jose Fernandez-Triana; institutionID: Canadian National Collection of Insects, Arachnids and Nematodes

e. country: Canada; stateProvince: Manitoba; county: Churchill; municipality: 16 km SW Churchill, Churchill River Pump House; verbatimLatitude: 58.626; verbatimLongitude: -94.229; verbatimEventDate: 07-Jul-2007; lifeStage: Adult; catalogNumber: 07PROBE-20921; otherCatalogNumbers: ASWAT273-08; identifiedBy: Jose FernandezTriana; institutionID: Biodiversity Institute of Ontario

Notes: Previously, this species was known to be widely distributed in the Palaearctic, with one record from tropical Africa (Yu et al. 2012). It was recently recorded from three provinces in Canada (BC, MB, SK) by Fernandez-Triana et al. (2014). A new provincial record (NL) is added here, altogether with detailed information about the distribution of this species in Canada, based on examined specimens from the BIO and CNC collections. Data for those specimens was extracted from BOLD.

\section{Cotesia crambi (Weed, 1887)}

\section{Materials}

a. $\quad$ country: United States; stateProvince: New Jersey; locality: Metuchen; verbatimEventDate: 19-Sep-1973; lifeStage: Adult; catalogNumber: CNCHYM 00401; recordedBy: Mailloux; otherCatalogNumbers: HYCNE211-11; identifiedBy: Jose 
Fernandez-Triana; institutionID: Canadian National Collection of Insects, Arachnids and Nematodes

b. $\quad$ country: Canada; stateProvince: Ontario; county: Paris; municipality: Holy Family School; locality: EQP-CLL-560; verbatimElevation: 250; verbatimLatitude: 43.207; verbatimLongitude: -80.391; verbatimEventDate: 27-Sep-2013; lifeStage: Adult; catalogNumber: BIOUG08665-E05; recordedBy: Anne Parkhill; otherCatalogNumbers: SMTPD4971-13; identifiedBy: Angela Telfer; institutionID: Biodiversity Institute of Ontario

c. country: Canada; stateProvince: Ontario; county: Leeds and Grenville; municipality: Elizabethtown-Kitley; locality: 4452 Rowsome Rd., Elizabethtown; verbatimElevation: 112; verbatimLatitude: 44.621; verbatimLongitude: -75.773; verbatimEventDate: 15Aug-2010; lifeStage: Adult; catalogNumber: BIOUG01258-H06; recordedBy: James Sones; otherCatalogNumbers: JSHYO850-11; identifiedBy: Angela Telfer; institutionID: Biodiversity Institute of Ontario

d. country: Canada; stateProvince: Ontario; county: Ottawa; locality: Mixed forest near Manotick; verbatimLatitude: 45.235; verbatimLongitude: -75.624; lifeStage: Adult; catalogNumber: CAM0542; recordedBy: A. Bennett; otherCatalogNumbers:

CNCHZ1162-09; identifiedBy: Jose Fernandez-Triana; institutionID: Canadian National Collection of Insects, Arachnids and Nematodes

e. country: Canada; stateProvince: Ontario; county: Wellington County; municipality: Guelph; Iocality: Biodiversity Institute of Ontario; verbatimElevation: 335; verbatimLatitude: 43.528; verbatimLongitude: -80.229; verbatimEventDate: 27-Jun-2013; lifeStage: Adult; catalogNumber: BIOUG08742-H10; recordedBy: BIO Collections Staff; otherCatalogNumbers: MBIOE759-13; identifiedBy: Angela Telfer; institutionID:

Biodiversity Institute of Ontario

f. country: United States; stateProvince: New Jersey; locality: Metuchen, Red Fescue; verbatimEventDate: 01-Jul-1974; lifeStage: Adult; catalogNumber: CNCHYM 00404; recordedBy: H. T. Streu; otherCatalogNumbers: HYCNE214-11; identifiedBy: Jose Fernandez-Triana; institutionID: Canadian National Collection of Insects, Arachnids and Nematodes

g. $\quad$ country: United States; stateProvince: New Jersey; locality: Metuchen; verbatimEventDate: 19-Sep-1973; lifeStage: Adult; catalogNumber: CNCHYM 00403; otherCatalogNumbers: HYCNE213-11; identifiedBy: Jose Fernandez-Triana; institutionID: Canadian National Collection of Insects, Arachnids and Nematodes

h. country: United States; stateProvince: New Jersey; locality: Metuchen; verbatimEventDate: 07-Sep-1973; lifeStage: Adult; catalogNumber: CNCHYM 00402; otherCatalogNumbers: HYCNE212-11; identifiedBy: Jose Fernandez-Triana; institutionID: Canadian National Collection of Insects, Arachnids and Nematodes

i. country: Canada; stateProvince: Ontario; county: Ottawa; locality: Ottwa city garden; verbatimLatitude: 45.356; verbatimLongitude: -75.707; lifeStage: Adult; catalogNumber: CAM1034; recordedBy: H. Goulet; otherCatalogNumbers: CNCHX164-09; identifiedBy: Jose Fernandez-Triana; institutionID: Canadian National Collection of Insects, Arachnids and Nematodes

j. $\quad$ country: Canada; stateProvince: Ontario; county: Point Pelee NP; municipality: Cactus Field; verbatimElevation: 168; verbatimLatitude: 41.939; verbatimLongitude: -82.516; verbatimEventDate: 15-Jun-2012; lifeStage: Adult; catalogNumber: BIOUG03438-B06; recordedBy: Heidi Brown; otherCatalogNumbers: CNPPD2118-12; identifiedBy: Angela Telfer; institutionID: Biodiversity Institute of Ontario

k. country: Canada; stateProvince: Ontario; county: Ottawa; locality: Ottwa city garden; verbatimLatitude: 45.356; verbatimLongitude: -75.707; lifeStage: Adult; catalogNumber: CAM0893; recordedBy: H. Goulet; otherCatalogNumbers: CNCHX023-09; identifiedBy: 
Jose Fernandez-Triana; institutionID: Canadian National Collection of Insects, Arachnids and Nematodes

I. country: Canada; stateProvince: Ontario; county: Point Pelee NP; municipality: Cactus Field; verbatimElevation: 168; verbatimLatitude: 41.939; verbatimLongitude: -82.516; verbatimEventDate: 29-Jun-2012; lifeStage: Adult; catalogNumber: BIOUG03445-F01; recordedBy: Tyler Peters; otherCatalogNumbers: CNPPE479-12; identifiedBy: Angela Telfer; institutionID: Biodiversity Institute of Ontario

Notes: This species has been recorded mainly from northeast and central United States (Yu et al. 2012, Fernandez-Triana et al. 2014). Until now it was only known from two localities in Canada (ON, QC) (Fernandez-Triana 2010, Fernandez-Triana et al. 2014). Here we present new and detailed information about four additional localities in $\mathrm{ON}$, based on examined specimens from the BIO and CNC collections. Data for those specimens was extracted from BOLD.

\section{Dolichogenidea longicauda (Wesmael, 1837)}

\section{Materials}

a. country: Canada; stateProvince: British Columbia; locality: Burnaby; verbatimEventDate: 09-Jul-1978; lifeStage: Adult; catalogNumber: CNCHYM 01091; recordedBy: Doganlar; otherCatalogNumbers: HYCNE902-11; identifiedBy: Jose Fernandez-Triana; institutionID: Canadian National Collection of Insects, Arachnids and Nematodes

b. $\quad$ country: Canada; stateProvince: British Columbia; locality: New Westminster; verbatimEventDate: 05-May-1978; lifeStage: Adult; catalogNumber: CNCHYM 01093; otherCatalogNumbers: HYCNE904-11; identifiedBy: Jose Fernandez-Triana; institutionID: Canadian National Collection of Insects, Arachnids and Nematodes

c. country: Canada; stateProvince: British Columbia; locality: Burnaby; verbatimEventDate: 01-Jul-1977; lifeStage: Adult; catalog Number: CNCHYM 01089; recordedBy: Doganlar; otherCatalogNumbers: HYCNE900-11; identifiedBy: Jose Fernandez-Triana; institutionID: Canadian National Collection of Insects, Arachnids and Nematodes

d. country: Canada; stateProvince: British Columbia; locality: Burnaby; verbatimEventDate: 01-Jul-1977; lifeStage: Adult; catalogNumber: CNCHYM 00216; recordedBy: Doganlar; otherCatalogNumbers: HYCNE026-11; identifiedBy: Jose Fernandez-Triana; institutionID: Canadian National Collection of Insects, Arachnids and Nematodes

Notes: This species is widely distributed in the Palaearctic (Yu et al. 2012). It was recorded from one province in Canada (BC) by Fernandez-Triana (2010), FernándezTriana and Huber (2010), but no locality records were provided at the time. Here we present new information about the distribution of this species in Canada $(B C)$, as well as the first record of this species from the United States (WA) based on examined specimens from the $\mathrm{BIO}$ and $\mathrm{CNC}$ collections. Data for those specimens was extracted from BOLD.

\section{Hygroplitis melligaster (Provancher, 1886)}

\section{Materials}

a. $\quad$ country: Canada; stateProvince: New Brunswick; locality: Kouchibouguac N.P.; verbatimEventDate: 18-Aug-1978; lifeStage: Adult; catalogNumber: CNCHYM 01363; 
recordedBy: D. B. Lyons; otherCatalogNumbers: HYCNE1175-11; identifiedBy: Jose Fernandez-Triana; institutionID: Canadian National Collection of Insects, Arachnids and Nematodes

b. $\quad$ country: United States; stateProvince: North Carolina; locality: Wayah Bald; verbatimElevation: 1249.7; verbatimEventDate: 29-Jul-1957; lifeStage: Adult; catalogNumber: CNCHYM 01366; recordedBy: W. R. Richards; otherCatalogNumbers: HYCNE1178-11; identifiedBy: Jose Fernandez-Triana; institutionID: Canadian National Collection of Insects, Arachnids and Nematodes

c. Country: Canada; stateProvince: Ontario; locality: Kemptville; verbatimEventDate: 28Jul-1983; lifeStage: Adult; catalogNumber: CNCHYM 01375; recordedBy: W. Mason; otherCatalogNumbers: HYCNE1187-11; identifiedBy: Jose Fernandez-Triana; institutionID: Canadian National Collection of Insects, Arachnids and Nematodes

d. country: Canada; stateProvince: Ontario; locality: 2 km SW of Innisville; verbatimEventDate: 26-Jun-1991; lifeStage: Adult; catalogNumber: GOU 0314; recordedBy: Sharkey \& Read; otherCatalogNumbers: CNCBA789-10; identifiedBy: Jose Fernandez-Triana; institutionID: Canadian National Collection of Insects, Arachnids and Nematodes

e. $\quad$ country: Canada; stateProvince: Nova Scotia; locality: Lockeport; verbatimEventDate: 09Aug-1958; lifeStage: Adult; catalogNumber: CNCHYM 01365; recordedBy: J. R. Vockeroth; otherCatalogNumbers: HYCNE1177-11; identifiedBy: Jose Fernandez-Triana; institutionID: Canadian National Collection of Insects, Arachnids and Nematodes

f. country: Canada; stateProvince: Prince Edward Island; locality: Ten Mile House, salt marsh; verbatimLatitude: 46.313; verbatimLongitude: -62.981; verbatimEventDate: 23Jul-2008; lifeStage: Adult; catalogNumber: CAM0473; recordedBy: H. Goulet, A. Badiss, C. Boudeault; otherCatalogNumbers: $\mathrm{CNCHZ663-09}$; identifiedBy: Jose FernandezTriana; institutionID: Canadian National Collection of Insects, Arachnids and Nematodes

g. country: Canada; stateProvince: New Brunswick; locality: St. Andrews; verbatimEventDate: 08-Aug-1959; lifeStage: Adult; catalogNumber: CNCHYM 01369; recordedBy: G. E. Shewell; otherCatalogNumbers: HYCNE1181-11; identifiedBy: Jose Fernandez-Triana; institutionID: Canadian National Collection of Insects, Arachnids and Nematodes

h. country: Canada; stateProvince: Prince Edward Island; locality: Ten Mile House, salt marsh; verbatimLatitude: 46.313; verbatimLongitude: -62.981; verbatimEventDate: 23Jul-2008; lifeStage: Adult; catalogNumber: CAM0470; recordedBy: H. Goulet, A. Badiss, C. Boudeault; otherCatalogNumbers: $\mathrm{CNCHZ660-09}$; identifiedBy: Jose FernandezTriana; institutionID: Canadian National Collection of Insects, Arachnids and Nematodes

i. country: Canada; stateProvince: Prince Edward Island; locality: Ten Mile House, salt marsh; verbatimLatitude: 46.313; verbatimLongitude: -62.981; verbatimEventDate: 23Jul-2008; lifeStage: Adult; catalogNumber: CAM0471; recordedBy: H. Goulet, A. Badiss, C. Boudeault; otherCatalogNumbers: CNCHZ661-09; identifiedBy: Jose FernandezTriana; institutionID: Canadian National Collection of Insects, Arachnids and Nematodes

j. country: Canada; stateProvince: Prince Edward Island; locality: Ten Mile House, salt marsh; verbatimLatitude: 46.313; verbatimLongitude: -62.981; verbatimEventDate: 23Jul-2008; lifeStage: Adult; catalogNumber: CAM0468; recordedBy: H. Goulet, A. Badiss, C. Boudeault; otherCatalogNumbers: $\mathrm{CNCHZ658-09}$; identifiedBy: Jose FernandezTriana; institutionID: Canadian National Collection of Insects, Arachnids and Nematodes

k. country: United States; stateProvince: North Carolina; locality: Highlands; verbatimElevation: 1158.25; verbatimEventDate: 12-Jul-1957; lifeStage: Adult; catalogNumber: CNCHYM 01370; recordedBy: J. G. Chillcott; otherCatalogNumbers: 
HYCNE1182-11; identifiedBy: Jose Fernandez-Triana; institutionID: Canadian National Collection of Insects, Arachnids and Nematodes

I. country: Canada; stateProvince: Prince Edward Island; locality: Ten Mile House, salt marsh; verbatimLatitude: 46.313 ; verbatimLongitude: -62.981; verbatimEventDate: 23Jul-2008; lifeStage: Adult; catalogNumber: CAM0463; recordedBy: H. Goulet, A. Badiss, C. Boudeault; otherCatalogNumbers: $\mathrm{CNCHZ653-09;}$; identifiedBy: Jose FernandezTriana; institutionID: Canadian National Collection of Insects, Arachnids and Nematodes

m. country: Canada; stateProvince: Prince Edward Island; locality: Ten Mile House, salt marsh; verbatimLatitude: 46.313; verbatimLongitude: -62.981; verbatimEventDate: 23Jul-2008; lifeStage: Adult; catalogNumber: CAM0465; recordedBy: H. Goulet, A. Badiss, C. Boudeault; otherCatalogNumbers: CNCHZ655-09; identifiedBy: Jose FernandezTriana; institutionID: Canadian National Collection of Insects, Arachnids and Nematodes

n. country: Canada; stateProvince: Prince Edward Island; locality: Ten Mile House, salt marsh; verbatimLatitude: 46.313; verbatimLongitude: -62.981; verbatimEventDate: 23Jul-2008; lifeStage: Adult; catalogNumber: CAM0464; recordedBy: H. Goulet, A. Badiss,

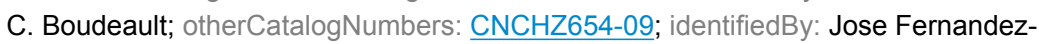
Triana; institutionID: Canadian National Collection of Insects, Arachnids and Nematodes

o. $\quad$ country: Canada; stateProvince: Manitoba; locality: Brandon; verbatimEventDate: 14Aug-1958; lifeStage: Adult; catalogNumber: CNCHYM 01368; recordedBy: R. B. Madge; otherCatalogNumbers: HYCNE1180-11; identifiedBy: Jose Fernandez-Triana; institutionID: Canadian National Collection of Insects, Arachnids and Nematodes

p. country: Canada; stateProvince: Ontario; county: St. Lawrence Islands National Park; locality: Grenadier Island Centre; verbatimEventDate: 29-Jul-1975; lifeStage: Adult; catalogNumber: CNCHYM 01367; recordedBy: McMillan; otherCatalogNumbers: HYCNE1179-11; identifiedBy: Jose Fernandez-Triana; institutionID: Canadian National Collection of Insects, Arachnids and Nematodes

q. country: Canada; stateProvince: Prince Edward Island; locality: Ten Mile House, salt marsh; verbatimLatitude: 46.313; verbatimLongitude: -62.981; verbatimEventDate: 23Jul-2008; lifeStage: Adult; catalog Number: CAM0462; recordedBy: H. Goulet, A. Badiss, C. Boudeault; otherCatalogNumbers: $\mathrm{CNCHZ652-09;}$; identifiedBy: Jose FernandezTriana; institutionID: Canadian National Collection of Insects, Arachnids and Nematodes

r. $\quad$ country: United States; stateProvince: New York; locality: Paul Smiths, Franklin Co.; verbatimEventDate: 20-Jul-1962; lifeStage: Adult; catalogNumber: CNCHYM 01371; recordedBy: J. R. Vockeroth; otherCatalogNumbers: HYCNE1183-11; identifiedBy: Jose Fernandez-Triana; institutionID: Canadian National Collection of Insects, Arachnids and Nematodes

s. $\quad$ country: Canada; stateProvince: Prince Edward Island; locality: Ten Mile House, salt marsh; verbatimLatitude: 46.313; verbatimLongitude: -62.981; verbatimEventDate: 23Jul-2008; lifeStage: Adult; catalogNumber: CAM0469; recordedBy: H. Goulet, A. Badiss, C. Boudeault; otherCatalogNumbers: $\mathrm{CNCHZ659-09;}$; identifiedBy: Jose FernandezTriana; institutionID: Canadian National Collection of Insects, Arachnids and Nematodes

t. Country: Canada; stateProvince: Ontario; locality: Aylmer West; verbatimEventDate: 22Jul-1972; lifeStage: Adult; catalogNumber: CNCHYM 01376; otherCatalogNumbers: HYCNE1188-11; identifiedBy: Jose Fernandez-Triana; institutionID: Canadian National Collection of Insects, Arachnids and Nematodes

u. $\quad$ country: Canada; stateProvince: Ontario; locality: Stittsville; verbatimEventDate: 22Aug-1963; lifeStage: Adult; catalogNumber: CNCHYM 01364; recordedBy: W. R. M. Mason; otherCatalogNumbers: HYCNE1176-11; identifiedBy: Jose Fernandez-Triana; institutionID: Canadian National Collection of Insects, Arachnids and Nematodes 
v. country: Canada; stateProvince: Ontario; locality: Rondeau Prov. Pk.; verbatimEventDate: 31-Jul-1972; lifeStage: Adult; catalogNumber: CNCHYM 01377; otherCatalogNumbers: HYCNE1189-11; identifiedBy: Jose Fernandez-Triana; institutionID: Canadian National Collection of Insects, Arachnids and Nematodes

w. $\quad$ country: Canada; stateProvince: Ontario; county: Ottawa; locality: Mer Bleue; verbatimEventDate: 09-Jul-1982; lifeStage: Adult; catalogNumber: CNCHYM 01378; recordedBy: L. Dumouchel; otherCatalogNumbers: HYCNE1190-11; identifiedBy: Jose Fernandez-Triana; institutionID: Canadian National Collection of Insects, Arachnids and Nematodes

x. country: Canada; stateProvince: Ontario; county: Leeds and Grenville; municipality: Elizabethtown-Kitley; locality: 4452 Rowsome Rd., Elizabethtown; verbatimElevation: 112; verbatimLatitude: 44.621; verbatimLongitude: -75.773; verbatimEventDate: 17 Jul-2010; lifeStage: Adult; catalogNumber: BIOUG01082-E12; recordedBy: James Sones; otherCatalogNumbers: JSHYN833-11; identifiedBy: Jose Fernandez-Triana; institutionID: Biodiversity Institute of Ontario

y. $\quad$ country: Canada; stateProvince: Prince Edward Island; locality: Ten Mile House, salt marsh; verbatimLatitude: 46.313; verbatimLongitude: -62.981; verbatimEventDate: 23Jul-2008; lifeStage: Adult; catalogNumber: CAM0474; recordedBy: H. Goulet, A. Badiss, C. Boudeault; otherCatalogNumbers: $\mathrm{CNCHZ664-09}$; identifiedBy: Jose FernandezTriana; institutionID: Canadian National Collection of Insects, Arachnids and Nematodes

z. country: Canada; stateProvince: Prince Edward Island; locality: Ten Mile House, salt marsh; verbatimLatitude: 46.313; verbatimLongitude: -62.981; verbatimEventDate: 23Jul-2008; lifeStage: Adult; catalogNumber: CAM0466; recordedBy: H. Goulet, A. Badiss, C. Boudeault; otherCatalogNumbers: $\mathrm{CNCHZ656-09;}$; identifiedBy: Jose FernandezTriana; institutionID: Canadian National Collection of Insects, Arachnids and Nematodes

aa. country: Canada; stateProvince: Prince Edward Island; locality: Ten Mile House, salt marsh; verbatimLatitude: 46.313; verbatimLongitude: -62.981; verbatimEventDate: 23Jul-2008; lifeStage: Adult; catalogNumber: CAM0472; recordedBy: H. Goulet, A. Badiss, C. Boudeault; otherCatalogNumbers: CNCHZ662-09; identifiedBy: Jose FernandezTriana; institutionID: Canadian National Collection of Insects, Arachnids and Nematodes ab. $\quad$ country: Canada; stateProvince: Prince Edward Island; locality: Ten Mile House, salt marsh; verbatimLatitude: 46.313; verbatimLongitude: -62.981; verbatimEventDate: 23Jul-2008; lifeStage: Adult; catalogNumber: CAM0467; recordedBy: H. Goulet, A. Badiss,

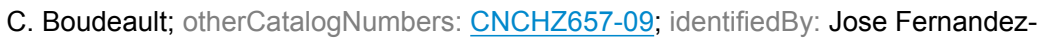
Triana; institutionID: Canadian National Collection of Insects, Arachnids and Nematodes

Notes: This species is relatively widely distributed in northeast North America, extending up to central Canada (Yu et al. 2012). It was recorded from five provinces in Canada (MB, NB, NS, ON, QC) by Fernandez-Triana (2010), but no locality records were provided at the time. Here we present additional information about the distribution of the species in Canada, including one new provincial record (PE), based on examined specimens from the $\mathrm{BIO}$ and $\mathrm{CNC}$ collections. Data for those specimens was extracted from BOLD.

\section{Pholetesor bedelliae (Viereck, 1911)}

\section{Materials}

a. country: Canada; stateProvince: Ontario; county: Ottawa; locality: Ottawa city garden; verbatimLatitude: 45.356; verbatimLongitude: -75.707; verbatimEventDate: 30-May-2007; 
lifeStage: Adult; catalogNumber: CAM0278; recordedBy: H. Goulet; otherCatalogNumbers: CNCHZ468-09; identifiedBy: Jose Fernandez-Triana; institutionID: Canadian National Collection of Insects, Arachnids and Nematodes

b. $\quad$ country: Canada; stateProvince: Alberta; county: Banff NP; municipality: Storm Mountain; locality: Adjacent to train track and Bow River; verbatimElevation: 1462; verbatimLatitude: 51.282; verbatimLongitude: -115.944; verbatimEventDate: 25-Jul-2012; lifeStage: Adult; catalogNumber: BIOUG04442-A12; recordedBy: BIOBus 2012; otherCatalogNumbers: SSBAD5451-13; institutionID: Biodiversity Institute of Ontario

C. $\quad$ country: Canada; stateProvince: Yukon Territory; locality: Fish Lake road; verbatimElevation: 1107; verbatimLatitude: 60.688; verbatimLongitude: -135.238; verbatimEventDate: 05-Jul-2006; lifeStage: Adult; catalogNumber: HYM00000958; recordedBy: Goulet \& Boudrealt; otherCatalogNumbers: DSWAS929-07; identifiedBy: Jose Fernandez-Triana; institutionID: Canadian National Collection of Insects, Arachnids and Nematodes

d. $\quad$ country: United States; stateProvince: Alaska; locality: Anderson, Hwy 3; verbatimElevation: 235; verbatimLatitude: 64.295 ; verbatimLongitude: -149.084 ; verbatimEventDate: 18-Jul-2009; lifeStage: Adult; catalogNumber: $\mathrm{CNCH} 3205$; recordedBy: Goulet \& Bouderault; otherCatalogNumbers: CNCHV275-10; identifiedBy: Jose Fernandez-Triana; institutionID: Canadian National Collection of Insects, Arachnids and Nematodes

e. country: Canada; stateProvince: Manitoba; county: Churchill; locality: 26 km SE Churchill, Twin Lakes Burn site; verbatimElevation: 38; verbatimLatitude: 58.617; verbatimLongitude: -93.812; verbatimEventDate: 19-Aug-2006; lifeStage: Adult; catalogNumber: HYM00000818; otherCatalogNumbers: DSWAS761-07; identifiedBy: Jose Fernandez-Triana; institutionID: Canadian National Collection of Insects, Arachnids and Nematodes

f. country: United States; stateProvince: Alaska; locality: Anderson, Hwy 3; verbatimElevation: 235; verbatimLatitude: 64.295 ; verbatimLongitude: -149.084 ; verbatimEventDate: 18-Jul-2009; lifeStage: Adult; catalogNumber: CNCH3212; recordedBy: Goulet \& Bouderault; otherCatalogNumbers: CNCHV282-10; identifiedBy: Jose Fernandez-Triana; institutionID: Canadian National Collection of Insects, Arachnids and Nematodes

g. country: Canada; stateProvince: British Columbia; county: 10 km W Kamloops; municipality: New Afton Mine; locality: Wetland, Site 1; verbatimElevation: 759; verbatimLatitude: 50.643; verbatimLongitude: -120.516; verbatimEventDate: 11-Jul-2013; lifeStage: Adult; catalogNumber: BIOUG07357-A10; recordedBy: Chrystal Simon; otherCatalogNumbers: NGNAC1789-13; institutionID: Biodiversity Institute of Ontario

h. $\quad$ country: Canada; stateProvince: Yukon Territory; locality: Pelly Crossing; verbatimElevation: 495; verbatimLatitude: 62.831; verbatimLongitude: -136.585 ; verbatimEventDate: 16-Jul-2006; lifeStage: Adult; catalogNumber: HYM00000994; recordedBy: Goulet \& Boudrealt; otherCatalogNumbers: DSWAS965-07; identifiedBy: Jose Fernandez-Triana; institutionID: Canadian National Collection of Insects, Arachnids and Nematodes

i. country: Canada; stateProvince: Yukon Territory; locality: Champagne; verbatimElevation: 733; verbatimLatitude: 60.790; verbatimLongitude: -136.437; verbatimEventDate: 08Jul-2006; lifeStage: Adult; catalogNumber: HYM00000974; recordedBy: Goulet \& Boudrealt; otherCatalogNumbers: DSWAS945-07; identifiedBy: Jose Fernandez-Triana; institutionID: Canadian National Collection of Insects, Arachnids and Nematodes

j. $\quad$ country: Canada; stateProvince: Yukon Territory; locality: Pelly Crossing; verbatimElevation: 495; verbatimLatitude: 62.831; verbatimLongitude: -136.585; 
verbatimEventDate: 15-Jul-2006; lifeStage: Adult; catalogNumber: HYM00000953; recordedBy: Goulet \& Boudrealt; otherCatalogNumbers: DSWAS924-07; identifiedBy: Jose Fernandez-Triana; institutionID: Canadian National Collection of Insects, Arachnids and Nematodes

k. country: Canada; stateProvince: Manitoba; county: Churchill; locality: unspecified locality; verbatimLatitude: 58.740; verbatimLongitude: -93.820; verbatimEventDate: 17-Jul-1949; lifeStage: Adult; catalogNumber: HYM00000821.2; recordedBy: R.Gwatkin; otherCatalogNumbers: ASWAY564-08; identifiedBy: Jose Fernandez-Triana; institutionID: Canadian National Collection of Insects, Arachnids and Nematodes

I. country: Canada; stateProvince: Ontario; locality: Faleyet; verbatimEventDate: 07Sep-1963; lifeStage: Adult; catalogNumber: CNCHYM 03136; recordedBy: F. I. S.; otherCatalogNumbers: HYCNE1821-11; identifiedBy: J. Whitfield; institutionID: Canadian National Collection of Insects, Arachnids and Nematodes

m. country: Canada; stateProvince: Manitoba; county: Churchill; locality: unspecified locality; verbatimLatitude: 58.740; verbatimLongitude: -93.820; verbatimEventDate: 17-Jul-1949; lifeStage: Adult; catalogNumber: HYM00000821; recordedBy: R.Gwatkin; otherCatalogNumbers: DSWAS764-07; identifiedBy: Jose Fernandez-Triana; institutionID: Canadian National Collection of Insects, Arachnids and Nematodes

n. country: Canada; stateProvince: Yukon Territory; locality: Champagne; verbatimElevation: 733; verbatimLatitude: 60.790; verbatimLongitude: -136.437; verbatimEventDate: 08Jul-2006; lifeStage: Adult; catalogNumber: HYM00000941; recordedBy: Goulet \& Boudrealt; otherCatalogNumbers: DSWAS912-07; identifiedBy: Jose Fernandez-Triana; institutionID: Canadian National Collection of Insects, Arachnids and Nematodes

o. $\quad$ country: United States; stateProvince: Alaska; locality: Anderson, Hwy 3; verbatimElevation: 235; verbatimLatitude: 64.295 ; verbatimLongitude: -149.084 ; verbatimEventDate: 18-Jul-2009; lifeStage: Adult; catalogNumber: $\mathrm{CNCH} 3209$; recordedBy: Goulet \& Bouderault; otherCatalogNumbers: CNCHV279-10; identifiedBy: Jose Fernandez-Triana; institutionID: Canadian National Collection of Insects, Arachnids and Nematodes

p. $\quad$ country: United States; stateProvince: Alaska; locality: Sutton on Glen Highway; verbatimElevation: 188; verbatimLatitude: 61.711; verbatimLongitude: -148.910; verbatimEventDate: 22-Jul-2009; lifeStage: Adult; catalogNumber: $\mathrm{CNCH} 3215$; recordedBy: Goulet \& Bouderault; otherCatalogNumbers: CNCHV380-10; identifiedBy: Jose Fernandez-Triana; institutionID: Canadian National Collection of Insects, Arachnids and Nematodes

q. country: Canada; stateProvince: British Columbia; locality: 5 Miles West of Lumby; verbatimEventDate: 26-May-1958; lifeStage: Adult; catalogNumber: CNCHYM 03143; recordedBy: F. I. S.; otherCatalogNumbers: HYCNE1828-11; identifiedBy: J. Whitfield; institutionID: Canadian National Collection of Insects, Arachnids and Nematodes

r. country: Canada; stateProvince: Yukon Territory; locality: Pelly Crossing; verbatimElevation: 495; verbatimLatitude: 62.831; verbatimLongitude: -136.585 ; verbatimEventDate: 16-Jul-2006; lifeStage: Adult; catalogNumber: HYM00000996; recordedBy: Goulet \& Boudrealt; otherCatalogNumbers: DSWAS967-07; identifiedBy: Jose Fernandez-Triana; institutionID: Canadian National Collection of Insects, Arachnids and Nematodes

s. $\quad$ country: United States; stateProvince: Alaska; locality: Delta Junction; verbatimElevation: 315; verbatimLatitude: 64.044; verbatimLongitude: -145.524; verbatimEventDate: 20 Jul-2009; lifeStage: Adult; catalogNumber: CNCH0955; recordedBy: Goulet \& Boudreault; otherCatalogNumbers: CNCHX970-09; identifiedBy: Jose Fernandez-Triana; institutionID: Canadian National Collection of Insects, Arachnids and Nematodes 
t. country: Canada; stateProvince: Yukon Territory; locality: Pelly Crossing; verbatimElevation: 495; verbatimLatitude: 62.831 ; verbatimLongitude: -136.585; verbatimEventDate: 15-Jul-2006; lifeStage: Adult; catalogNumber: HYM00000948; recordedBy: Goulet \& Boudrealt; otherCatalogNumbers: DSWAS919-07; identifiedBy: Jose Fernandez-Triana; institutionID: Canadian National Collection of Insects, Arachnids and Nematodes

u. $\quad$ country: United States; stateProvince: Alaska; locality: Anderson, Hwy 3; verbatimElevation: 235; verbatimLatitude: 64.295 ; verbatimLongitude: -149.084 ; verbatimEventDate: 18-Jul-2009; lifeStage: Adult; catalogNumber: $\mathrm{CNCH} 3208$; recordedBy: Goulet \& Bouderault; otherCatalogNumbers: CNCHV278-10; identifiedBy: Jose Fernandez-Triana; institutionID: Canadian National Collection of Insects, Arachnids and Nematodes

v. country: Canada; stateProvince: Yukon Territory; locality: Champagne; verbatimElevation: 733; verbatimLatitude: 60.790; verbatimLongitude: -136.437; verbatimEventDate: 08Jul-2006; lifeStage: Adult; catalogNumber: HYM00000986; recordedBy: Goulet \& Boudrealt; otherCatalogNumbers: DSWAS957-07; identifiedBy: Jose Fernandez-Triana; institutionID: Canadian National Collection of Insects, Arachnids and Nematodes

w. country: Canada; stateProvince: Manitoba; county: Churchill; locality: Mile 505, Hudson Bay Railway; verbatimEventDate: 16-Jul-1952; lifeStage: Adult; catalogNumber: HYM00000820; recordedBy: J.G.Chillcot; otherCatalogNumbers: DSWAS763-07; identifiedBy: Jose Fernandez-Triana; institutionID: Canadian National Collection of Insects, Arachnids and Nematodes

x. country: Canada; stateProvince: Yukon Territory; locality: Champagne; verbatimElevation: 733; verbatimLatitude: 60.790; verbatimLongitude: -136.437; verbatimEventDate: 08Jul-2006; lifeStage: Adult; catalogNumber: HYM00000971; recordedBy: Goulet \& Boudrealt; otherCatalogNumbers: DSWAS942-07; identifiedBy: Jose Fernandez-Triana; institutionID: Canadian National Collection of Insects, Arachnids and Nematodes

y. $\quad$ country: Canada; stateProvince: Yukon Territory; locality: Pelly Crossing; verbatimElevation: 495; verbatimLatitude: 62.831 ; verbatimLongitude: -136.585 ; verbatimEventDate: 15-Jul-2006; lifeStage: Adult; catalogNumber: HYM00000955; recordedBy: Goulet \& Boudrealt; otherCatalogNumbers: DSWAS926-07; identifiedBy: Jose Fernandez-Triana; institutionID: Canadian National Collection of Insects, Arachnids and Nematodes

z. $\quad$ country: United States; stateProvince: Alaska; locality: Sutton on Glen Highway; verbatimElevation: 188; verbatimLatitude: 61.711; verbatimLongitude: -148.910; verbatimEventDate: 22-Jul-2009; lifeStage: Adult; catalogNumber: CNCH3306; recordedBy: Goulet \& Bouderault; otherCatalogNumbers: CNCHV375-10; identifiedBy: Jose Fernandez-Triana; institutionID: Canadian National Collection of Insects, Arachnids and Nematodes

aa. country: Canada; stateProvince: Yukon Territory; locality: Champagne; verbatimElevation: 733; verbatimLatitude: 60.790; verbatimLongitude: -136.437; verbatimEventDate: 08Jul-2006; lifeStage: Adult; catalogNumber: HYM00000972; recordedBy: Goulet \& Boudrealt; otherCatalogNumbers: DSWAS943-07; identifiedBy: Jose Fernandez-Triana; institutionID: Canadian National Collection of Insects, Arachnids and Nematodes

ab. country: Canada; stateProvince: Yukon Territory; locality: Pelly Crossing; verbatimElevation: 495; verbatimLatitude: 62.831; verbatimLongitude: -136.585; verbatimEventDate: 15-Jul-2006; lifeStage: Adult; catalogNumber: HYM00000947; recordedBy: Goulet \& Boudrealt; otherCatalogNumbers: DSWAS918-07; identifiedBy: Jose Fernandez-Triana; institutionID: Canadian National Collection of Insects, Arachnids and Nematodes 
ac. country: United States; stateProvince: Alaska; locality: Sutton on Glen Highway; verbatimElevation: 188; verbatimLatitude: 61.711; verbatimLongitude: -148.910; verbatimEventDate: 22-Jul-2009; lifeStage: Adult; catalogNumber: CNCH3310; recordedBy: Goulet \& Bouderault; otherCatalogNumbers: CNCHV379-10; identifiedBy: Jose Fernandez-Triana; institutionID: Canadian National Collection of Insects, Arachnids and Nematodes

ad. country: Canada; stateProvince: Yukon Territory; locality: Pelly Crossing; verbatimElevation: 495; verbatimLatitude: 62.831; verbatimLongitude: -136.585; verbatimEventDate: 15-Jul-2006; lifeStage: Adult; catalogNumber: HYM00000931; recordedBy: Goulet \& Boudrealt; otherCatalogNumbers: DSWAS902-07; identifiedBy: Jose Fernandez-Triana; institutionID: Canadian National Collection of Insects, Arachnids and Nematodes

ae. country: Canada; stateProvince: Yukon Territory; locality: Champagne; verbatimElevation: 733; verbatimLatitude: 60.790; verbatimLongitude: -136.437; verbatimEventDate: 08Jul-2006; lifeStage: Adult; catalogNumber: HYM00000985; recordedBy: Goulet \& Boudrealt; otherCatalogNumbers: DSWAS956-07; identifiedBy: Jose Fernandez-Triana; institutionID: Canadian National Collection of Insects, Arachnids and Nematodes

af. $\quad$ country: United States; stateProvince: Alaska; locality: Anderson, Hwy 3; verbatimElevation: 235; verbatimLatitude: 64.295; verbatimLongitude: -149.084; verbatimEventDate: 18-Jul-2009; lifeStage: Adult; catalogNumber: CNCH3203; recordedBy: Goulet \& Bouderault; otherCatalogNumbers: CNCHV273-10; identifiedBy: Jose Fernandez-Triana; institutionID: Canadian National Collection of Insects, Arachnids and Nematodes

ag. country: United States; stateProvince: California; locality: Fallen Leaf, Eldorado co.; verbatimElevation: 1981.22; verbatimEventDate: 13-Jul-1961; lifeStage: Adult; catalogNumber: CNCHYM 03141; recordedBy: H. B. Poole; otherCatalogNumbers: HYCNE1826-11; identifiedBy: Jose Fernandez-Triana; institutionID: Canadian National Collection of Insects, Arachnids and Nematodes

ah. $\quad$ country: United States; stateProvince: Alaska; locality: Delta Junction; verbatimElevation: 315; verbatimLatitude: 64.044; verbatimLongitude: -145.524; verbatimEventDate: 20 Jul-2009; lifeStage: Adult; catalogNumber: CNCH0995; recordedBy: Goulet \& Boudreault; otherCatalogNumbers: CNCHX1010-09; identifiedBy: Jose Fernandez-Triana; institutionID: Canadian National Collection of Insects, Arachnids and Nematodes

ai. country: Canada; stateProvince: Yukon Territory; locality: Whitehorse, fallow field; verbatimElevation: 689; verbatimLatitude: 60.710; verbatimLongitude: -135.055; verbatimEventDate: 10-Jul-2006; lifeStage: Adult; catalogNumber: HYM00000331; recordedBy: Boudreault \& Goulet; otherCatalogNumbers: CNCHY059-07; identifiedBy: Jose Fernandez-Triana; institutionID: Canadian National Collection of Insects, Arachnids and Nematodes

aj. country: Canada; stateProvince: Yukon Territory; locality: Champagne; verbatimElevation: 733; verbatimLatitude: 60.790; verbatimLongitude: -136.437; verbatimEventDate: 08Jul-2006; lifeStage: Adult; catalogNumber: HYM00000939; recordedBy: Goulet \& Boudrealt; otherCatalogNumbers: DSWAS910-07; identifiedBy: Jose Fernandez-Triana; institutionID: Canadian National Collection of Insects, Arachnids and Nematodes

ak. country: Canada; stateProvince: Yukon Territory; locality: Champagne; verbatimElevation: 733; verbatimLatitude: 60.790; verbatimLongitude: -136.437; verbatimEventDate: 08Jul-2006; lifeStage: Adult; catalogNumber: HYM00000969; recordedBy: Goulet \& Boudrealt; otherCatalogNumbers: DSWAS940-07; identifiedBy: Jose Fernandez-Triana; institutionID: Canadian National Collection of Insects, Arachnids and Nematodes 
al. country: United States; stateProvince: Alaska; locality: Delta Junction; verbatimElevation: 315; verbatimLatitude: 64.044; verbatimLongitude: -145.524; verbatimEventDate: 20 Jul-2009; lifeStage: Adult; catalogNumber: CNCH0993; recordedBy: Goulet \& Boudreault; otherCatalogNumbers: CNCHX1008-09; identifiedBy: Jose Fernandez-Triana; institutionID: Canadian National Collection of Insects, Arachnids and Nematodes

am. country: Canada; stateProvince: New Brunswick; locality: Fredericton; verbatimEventDate: 09-Jul-1970; lifeStage: Adult; catalogNumber: CNCHYM 03145; recordedBy: C. M. Yoshimoto; otherCatalogNumbers: HYCNE1830-11; identifiedBy: J. Whitfield; institutionID: Canadian National Collection of Insects, Arachnids and Nematodes

an. country: Canada; stateProvince: Manitoba; county: Churchill; locality: 26 km SE Churchill, Twin Lakes Burn site; verbatimElevation: 38; verbatimLatitude: 58.617 ; verbatimLongitude: -93.812; verbatimEventDate: 19-Aug-2006; lifeStage: Adult; catalogNumber: HYM00000819; otherCatalogNumbers: DSWAS762-07; identifiedBy: Jose Fernandez-Triana; institutionID: Canadian National Collection of Insects, Arachnids and Nematodes

ao. country: Canada; stateProvince: British Columbia; locality: Langford; verbatimEventDate: 22-Jun-1962; lifeStage: Adult; catalogNumber: CNCHYM 03138; recordedBy: F. I. S.; otherCatalogNumbers: HYCNE1823-11; identifiedBy: J. Whitfield; institutionID: Canadian National Collection of Insects, Arachnids and Nematodes

ap. $\quad$ country: United States; stateProvince: Alaska; locality: Anderson, Hwy 3; verbatimElevation: 235; verbatimLatitude: 64.295 ; verbatimLongitude: -149.084 ; verbatimEventDate: 18-Jul-2009; lifeStage: Adult; catalogNumber: CNCH3204; recordedBy: Goulet \& Bouderault; otherCatalogNumbers: CNCHV274-10; identifiedBy: Jose Fernandez-Triana; institutionID: Canadian National Collection of Insects, Arachnids and Nematodes

aq. $\quad$ country: United States; stateProvince: Alaska; locality: Anderson, Hwy 3; verbatimElevation: 235; verbatimLatitude: 64.295 ; verbatimLongitude: -149.084 ; verbatimEventDate: 18-Jul-2009; lifeStage: Adult; catalogNumber: CNCH3213; recordedBy: Goulet \& Bouderault; otherCatalogNumbers: CNCHV283-10; identifiedBy: Jose Fernandez-Triana; institutionID: Canadian National Collection of Insects, Arachnids and Nematodes

ar. country: Canada; stateProvince: Ontario; county: Haldimond-Dunn Townline; municipality: Windy Bluff; verbatimElevation: 179; verbatimLatitude: 42.861 ; verbatimLongitude: -79.703; verbatimEventDate: 19-Sep-2010; lifeStage: Adult; catalogNumber: BIOUG00989-E07; recordedBy: Alex Smith; otherCatalogNumbers: ASGLF308-11; identifiedBy: Jose Fernandez-Triana; institutionID: Research Collection of M. Alex Smith

as. $\quad$ country: Canada; stateProvince: Ontario; county: Point Pelee NP; municipality: Cactus Field; verbatimElevation: 168; verbatimLatitude: 41.939; verbatimLongitude: -82.516; verbatimEventDate: 07-Sep-2012; lifeStage: Adult; catalogNumber: BIOUG03931-A10; recordedBy: H.Brown; otherCatalogNumbers: CNPPJ1263-12; institutionID: Biodiversity Institute of Ontario

at. country: United States; stateProvince: Alaska; locality: Delta Junction; verbatimElevation: 315; verbatimLatitude: 64.044; verbatimLongitude: -145.524; verbatimEventDate: 20 Jul-2009; lifeStage: Adult; catalogNumber: CNCH0965; recordedBy: Goulet \& Boudreault;

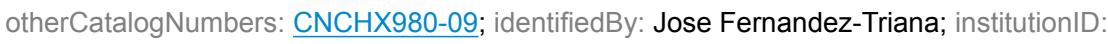
Canadian National Collection of Insects, Arachnids and Nematodes

au. country: Canada; stateProvince: Saskatchewan; locality: Grassland National Park; verbatimElevation: 830; verbatimLatitude: 49.074; verbatimLongitude: -106.529; verbatimEventDate: 04-Jun-2007; lifeStage: Adult; catalogNumber: HYM00001322; 
recordedBy: Goulet, Boudreault \& Fernandez; otherCatalogNumbers: ASWA608-08; identifiedBy: Jose Fernandez-Triana; institutionID: Canadian National Collection of Insects, Arachnids and Nematodes

av. country: Canada; stateProvince: Ontario; county: Almonte; locality: 5 km NW of Almonte, Hwy 49, Burnt Land, Alvar Prov. Park; verbatimLatitude: 45.255; verbatimLongitude: -76.140; verbatimEventDate: 29-May-2008; lifeStage: Adult; catalogNumber: CAM0437;

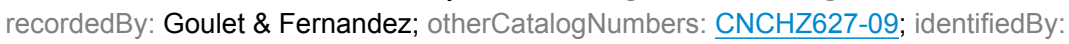
Jose Fernandez-Triana; institutionID: Canadian National Collection of Insects, Arachnids and Nematodes

aw. country: Canada; stateProvince: Yukon Territory; locality: Pelly Crossing; verbatimElevation: 495; verbatimLatitude: 62.831 ; verbatimLongitude: -136.585 ; verbatimEventDate: 15-Jul-2006; lifeStage: Adult; catalogNumber: HYM00000990; recordedBy: Goulet \& Boudrealt; otherCatalogNumbers: DSWAS961-07; identifiedBy: Jose Fernandez-Triana; institutionID: Canadian National Collection of Insects, Arachnids and Nematodes

ax. $\quad$ country: Canada; stateProvince: Ontario; locality: Macdiarmid; verbatimEventDate: 03Aug-1962; lifeStage: Adult; catalogNumber: CNCHYM 03139; recordedBy: F. I. S.; otherCatalogNumbers: HYCNE1824-11; identifiedBy: J. Whitfield; institutionID: Canadian National Collection of Insects, Arachnids and Nematodes

ay. country: Canada; stateProvince: Yukon Territory; locality: Champagne; verbatimElevation: 733; verbatimLatitude: 60.790; verbatimLongitude: -136.437; verbatimEventDate: 08Jul-2006; lifeStage: Adult; catalogNumber: HYM00000975; recordedBy: Goulet \& Boudrealt; otherCatalogNumbers: DSWAS946-07; identifiedBy: Jose Fernandez-Triana; institutionID: Canadian National Collection of Insects, Arachnids and Nematodes

az. $\quad$ country: United States; stateProvince: Alaska; locality: Anderson, Hwy 3; verbatimElevation: 235 ; verbatimLatitude: 64.295 ; verbatimLongitude: -149.084; verbatimEventDate: 18-Jul-2009; lifeStage: Adult; catalogNumber: $\mathrm{CNCH} 3206$; recordedBy: Goulet \& Bouderault; otherCatalogNumbers: CNCHV276-10; identifiedBy: Jose Fernandez-Triana; institutionID: Canadian National Collection of Insects, Arachnids and Nematodes

ba. $\quad$ country: Canada; stateProvince: Yukon Territory; locality: Pelly Crossing; verbatimElevation: 495; verbatimLatitude: 62.831 ; verbatimLongitude: -136.585 ; verbatimEventDate: 15-Jul-2006; lifeStage: Adult; catalogNumber: HYM00000935; recordedBy: Goulet \& Boudrealt; otherCatalogNumbers: DSWAS906-07; identifiedBy: Jose Fernandez-Triana; institutionID: Canadian National Collection of Insects, Arachnids and Nematodes

bb. country: United States; stateProvince: Alaska; locality: Delta Junction; verbatimElevation: 315; verbatimLatitude: 64.044; verbatimLongitude: -145.524; verbatimEventDate: 20 Jul-2009; lifeStage: Adult; catalogNumber: CNCH1008; recordedBy: Goulet \& Boudreault;

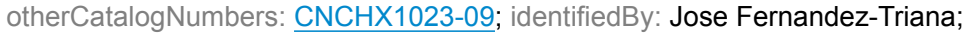
institutionID: Canadian National Collection of Insects, Arachnids and Nematodes

bc. country: Canada; stateProvince: Yukon Territory; locality: Champagne; verbatimElevation: 733; verbatimLatitude: 60.790; verbatimLongitude: -136.437; verbatimEventDate: 08Jul-2006; lifeStage: Adult; catalogNumber: HYM00000987; recordedBy: Goulet \& Boudrealt; otherCatalogNumbers: DSWAS958-07; identifiedBy: Jose Fernandez-Triana; institutionID: Canadian National Collection of Insects, Arachnids and Nematodes

bd. country: United States; stateProvince: Alaska; locality: Delta Junction; verbatimElevation: 315; verbatimLatitude: 64.044; verbatimLongitude: -145.524; verbatimEventDate: 20 Jul-2009; lifeStage: Adult; catalogNumber: CNCH0987; recordedBy: Goulet \& Boudreault; 
otherCatalogNumbers: CNCHX1002-09; identifiedBy: Jose Fernandez-Triana; institutionID: Canadian National Collection of Insects, Arachnids and Nematodes

be. $\quad$ country: Canada; stateProvince: Saskatchewan; county: Grasslands NP; municipality:

East Block badlands; verbatimElevation: 848; verbatimLatitude: 49.071 ; verbatimLongitude: -106.531; verbatimEventDate: 16-Jul-2008; lifeStage: Adult; catalogNumber: 08BBHYM-1368; recordedBy: J.Cossey, N.Jeffery, J.Straka; otherCatalogNumbers: BBHYM324-09; identifiedBy: Jose Fernandez-Triana; institutionID: Biodiversity Institute of Ontario

bf. country: Canada; stateProvince: Yukon Territory; locality: Champagne; verbatimElevation: 733; verbatimLatitude: 60.790; verbatimLongitude: -136.437; verbatimEventDate: 08Jul-2006; lifeStage: Adult; catalogNumber: HYM00000968; recordedBy: Goulet \& Boudrealt; otherCatalogNumbers: DSWAS939-07; identifiedBy: Jose Fernandez-Triana; institutionID: Canadian National Collection of Insects, Arachnids and Nematodes

bg. $\quad$ country: United States; stateProvince: California; locality: Los Angeles; verbatimEventDate: 01-Dec-1944; lifeStage: Adult; catalogNumber: CNCHYM 03144; otherCatalogNumbers: HYCNE1829-11; identifiedBy: Jose Fernandez-Triana; institutionID: Canadian National Collection of Insects, Arachnids and Nematodes

bh. $\quad$ country: Canada; stateProvince: Yukon Territory; locality: Pelly Crossing; verbatimElevation: 495; verbatimLatitude: 62.831 ; verbatimLongitude: -136.585 ; verbatimEventDate: 15-Jul-2006; lifeStage: Adult; catalogNumber: HYM00000936; recordedBy: Goulet \& Boudrealt; otherCatalogNumbers: DSWAS907-07; identifiedBy: Jose Fernandez-Triana; institutionID: Canadian National Collection of Insects, Arachnids and Nematodes

bi. $\quad$ country: Canada; stateProvince: Yukon Territory; locality: Pelly Crossing; verbatimElevation: 495; verbatimLatitude: 62.831; verbatimLongitude: -136.585 ; verbatimEventDate: 15-Jul-2006; lifeStage: Adult; catalogNumber: HYM00000951; recordedBy: Goulet \& Boudrealt; otherCatalogNumbers: DSWAS922-07; identifiedBy: Jose Fernandez-Triana; institutionID: Canadian National Collection of Insects, Arachnids and Nematodes

bj. $\quad$ country: Canada; stateProvince: Saskatchewan; locality: Grassland National Park; verbatimElevation: 830; verbatimLatitude: 49.074; verbatimLongitude: -106.529; verbatimEventDate: 04-Jun-2007; lifeStage: Adult; catalogNumber: HYM00001320; recordedBy: Goulet, Boudreault \& Fernandez; otherCatalogNumbers: ASWA606-08; identifiedBy: Jose Fernandez-Triana; institutionID: Canadian National Collection of Insects, Arachnids and Nematodes

bk. country: Canada; stateProvince: Manitoba; county: Churchill; locality: 26 km SE Churchill, Twin Lakes Burn site; verbatimElevation: 38; verbatimLatitude: 58.617 ; verbatimLongitude: -93.812; verbatimEventDate: 17-Aug-2006; lifeStage: Adult; catalogNumber: HYM00000920; otherCatalogNumbers: DSWAS863-07; identifiedBy: Jose Fernandez-Triana; institutionID: Canadian National Collection of Insects, Arachnids and Nematodes

bl. $\quad$ country: Canada; stateProvince: Ontario; locality: Hearst; verbatimEventDate: 03Aug-1971; lifeStage: Adult; catalogNumber: CNCHYM 03137; otherCatalogNumbers: HYCNE1822-11; identifiedBy: Jose Fernandez-Triana; institutionID: Canadian National Collection of Insects, Arachnids and Nematodes

bm. Country: Canada; stateProvince: Manitoba; county: Churchill; locality: Mile 505, Hudson Bay Railway; verbatimEventDate: 16-Jul-1952; lifeStage: Adult; catalogNumber: HYM00000820.2; recordedBy: J.G.Chillcot; otherCatalogNumbers: ASWAY563-08; identifiedBy: Jose Fernandez-Triana; institutionID: Canadian National Collection of Insects, Arachnids and Nematodes 
bn. country: Canada; stateProvince: Yukon Territory; locality: Champagne; verbatimElevation: 733; verbatimLatitude: 60.790; verbatimLongitude: -136.437; verbatimEventDate: 08Jul-2006; lifeStage: Adult; catalogNumber: HYM00000976; recordedBy: Goulet \& Boudrealt; otherCatalogNumbers: DSWAS947-07; identifiedBy: Jose Fernandez-Triana; institutionID: Canadian National Collection of Insects, Arachnids and Nematodes

bo. country: Canada; stateProvince: Yukon Territory; locality: Champagne; verbatimElevation: 733; verbatimLatitude: 60.790; verbatimLongitude: -136.437; verbatimEventDate: 08Jul-2006; lifeStage: Adult; catalogNumber: HYM00000943; recordedBy: Goulet \& Boudrealt; otherCatalogNumbers: DSWAS914-07; identifiedBy: Jose Fernandez-Triana; institutionID: Canadian National Collection of Insects, Arachnids and Nematodes

bp. $\quad$ country: Canada; stateProvince: Yukon Territory; locality: Pelly Crossing; verbatimElevation: 495; verbatimLatitude: 62.831; verbatimLongitude: -136.585 ; verbatimEventDate: 15-Jul-2006; lifeStage: Adult; catalogNumber: HYM00000934; recordedBy: Goulet \& Boudrealt; otherCatalogNumbers: DSWAS905-07; identifiedBy: Jose Fernandez-Triana; institutionID: Canadian National Collection of Insects, Arachnids and Nematodes

bq. Country: Canada; stateProvince: Yukon Territory; locality: Champagne; verbatimElevation: 733; verbatimLatitude: 60.790; verbatimLongitude: -136.437; verbatimEventDate: 08Jul-2006; lifeStage: Adult; catalogNumber: HYM00000938; recordedBy: Goulet \& Boudrealt; otherCatalogNumbers: DSWAS909-07; identifiedBy: Jose Fernandez-Triana; institutionID: Canadian National Collection of Insects, Arachnids and Nematodes

br. $\quad$ country: Canada; stateProvince: Yukon Territory; locality: Pelly Crossing; verbatimElevation: 495; verbatimLatitude: 62.831; verbatimLongitude: -136.585 ; verbatimEventDate: 15-Jul-2006; lifeStage: Adult; catalogNumber: HYM00000950; recordedBy: Goulet \& Boudrealt; otherCatalogNumbers: DSWAS921-07; identifiedBy: Jose Fernandez-Triana; institutionID: Canadian National Collection of Insects, Arachnids and Nematodes

bs. country: Canada; stateProvince: Yukon Territory; locality: Champagne; verbatimElevation: 733; verbatimLatitude: 60.790; verbatimLongitude: -136.437; verbatimEventDate: 08Jul-2006; lifeStage: Adult; catalogNumber: HYM00000956; recordedBy: Goulet \& Boudrealt; otherCatalogNumbers: DSWAS927-07; identifiedBy: Jose Fernandez-Triana; institutionID: Canadian National Collection of Insects, Arachnids and Nematodes

bt. country: Canada; stateProvince: Yukon Territory; locality: Champagne; verbatimElevation: 733; verbatimLatitude: 60.790; verbatimLongitude: -136.437; verbatimEventDate: 08Jul-2006; lifeStage: Adult; catalogNumber: HYM00000973; recordedBy: Goulet \& Boudrealt; otherCatalogNumbers: DSWAS944-07; identifiedBy: Jose Fernandez-Triana; institutionID: Canadian National Collection of Insects, Arachnids and Nematodes

bu. country: Canada; stateProvince: Yukon Territory; locality: Champagne; verbatimElevation: 733; verbatimLatitude: 60.790; verbatimLongitude: -136.437; verbatimEventDate: 08Jul-2006; lifeStage: Adult; catalogNumber: HYM00000970; recordedBy: Goulet \& Boudrealt; otherCatalogNumbers: DSWAS941-07; identifiedBy: Jose Fernandez-Triana; institutionID: Canadian National Collection of Insects, Arachnids and Nematodes

bv. country: Canada; stateProvince: Yukon Territory; locality: Champagne; verbatimElevation: 733; verbatimLatitude: 60.790; verbatimLongitude: -136.437; verbatimEventDate: 08Jul-2006; lifeStage: Adult; catalogNumber: HYM00000940; recordedBy: Goulet \& Boudrealt; otherCatalogNumbers: DSWAS911-07; identifiedBy: Jose Fernandez-Triana; institutionID: Canadian National Collection of Insects, Arachnids and Nematodes

bw. country: Canada; stateProvince: Newfoundland and Labrador; locality: Agric.Exp.Sta.St.John`s; verbatimEventDate: 20-Jul-1967; lifeStage: Adult; catalogNumber: CNCHYM 03142; recordedBy: J. F. McAlpine; otherCatalogNumbers: 
HYCNE1827-11; identifiedBy: Jose Fernandez-Triana; institutionID: Canadian National Collection of Insects, Arachnids and Nematodes

bx. country: Canada; stateProvince: Yukon Territory; locality: Champagne; verbatimElevation: 733; verbatimLatitude: 60.790; verbatimLongitude: -136.437; verbatimEventDate: 08Jul-2006; lifeStage: Adult; catalogNumber: HYM00000942; recordedBy: Goulet \& Boudrealt; otherCatalogNumbers: DSWAS913-07; identifiedBy: Jose Fernandez-Triana; institutionID: Canadian National Collection of Insects, Arachnids and Nematodes

by. $\quad$ country: United States; stateProvince: Colorado; locality: Muddy Pass Jackson Co.; verbatimElevation: 2682.27; verbatimEventDate: 15-Aug-1961; lifeStage: Adult; catalogNumber: CNCHYM 03140; recordedBy: B. Poole; otherCatalogNumbers: HYCNE1825-11; identifiedBy: Jose Fernandez-Triana; institutionID: Canadian National Collection of Insects, Arachnids and Nematodes

bz. country: Canada; stateProvince: Manitoba; county: Churchill; municipality: $26 \mathrm{~km} \mathrm{SE}$ Churchill, Twin Lakes; verbatimLatitude: 58.632; verbatimLongitude: -93.788; verbatimEventDate: 07-Jul-2007; lifeStage: Adult; catalogNumber: 07PROBE-20072; recordedBy: A.Renaud; otherCatalogNumbers: ASWA270-08; identifiedBy: Jose Fernandez-Triana; institutionID: Biodiversity Institute of Ontario

Notes: This species is widely distributed within the Nearctic (e.g., Whitfield 2006, Fernandez-Triana 2010, Yu et al. 2012, Fernandez-Triana et al. 2014), and is also reported from Finland (Hellén 1977), Peru (de Huiza 1995), and was introduced to Hawai (Yu et al. 2012). Here we present additional information about the distribution of this species in Canada, including one new provincial record (AB), based on examined specimens from the $\mathrm{BIO}$ and $\mathrm{CNC}$ collections. Data for those specimens was extracted from BOLD.

\section{Pholetesor viminetorum (Wesmael, 1837)}

\section{Materials}

a. country: United States; stateProvince: Colorado; locality: Doolittle Ranch Mt. Evans; verbatimElevation: 2987.08; verbatimEventDate: 09-Aug-1961; lifeStage: Adult; catalogNumber: CNCHYM 03217; recordedBy: S. M. Clark; otherCatalogNumbers: HYCNF002-11; identifiedBy: J. Whitfield; institutionID: Canadian National Collection of Insects, Arachnids and Nematodes

b. country: Canada; stateProvince: Manitoba; county: Churchill; locality: 23 km E Churchill, Fort Churchill; verbatimElevation: 27 ; verbatimLatitude: 58.734 ; verbatimLongitude: -93.789; verbatimEventDate: 18-Jul-1952; lifeStage: Adult; catalogNumber: HYM00000806; recordedBy: J.G.Chillcot; otherCatalogNumbers: DSWAS749-07; identifiedBy: Jose Fernandez-Triana; institutionID: Canadian National Collection of Insects, Arachnids and Nematodes

c. country: Canada; stateProvince: Manitoba; county: Churchill; locality: $23 \mathrm{~km}$ E Churchill, Ramsay Creek; verbatimElevation: 10; verbatimLatitude: 58.731; verbatimLongitude: -93.780; verbatimEventDate: 16-Jul-2007; lifeStage: Adult; catalogNumber: 07PROBE-22423; recordedBy: A.Renaud; otherCatalogNumbers: ASWAV964-08; identifiedBy: Jose Fernandez-Triana; institutionID: Biodiversity Institute of Ontario

d. country: Canada; stateProvince: Newfoundland and Labrador; county: Gros Morne NP; municipality: Lomond River Trail; locality: Balsam fir, larch, fern and wildflowers (Ladyslipper orchid); verbatimElevation: 35; verbatimLatitude: 49.429; verbatimLongitude: -57.741; verbatimEventDate: 19-Jul-2013; lifeStage: Adult; catalogNumber: 
BIOUG14184-G04; recordedBy: BIObus 2013; otherCatalogNumbers: SSGBB2792-14; identifiedBy: Angela Telfer; institutionID: Biodiversity Institute of Ontario

e. country: Canada; stateProvince: Manitoba; county: Churchill; locality: Fort Churchill; verbatimLatitude: 58.734; verbatimLongitude: -93.820; verbatimEventDate: 11-Jul-1952; lifeStage: Adult; catalogNumber: HYM00000809.2; recordedBy: J. G. Chillcot; otherCatalogNumbers: ASWAY600-08; identifiedBy: Jose Fernandez-Triana; institutionID: Canadian National Collection of Insects, Arachnids and Nematodes

f. country: Canada; stateProvince: Manitoba; county: Churchill; municipality: 12 km ESE Churchill, Launch Road; locality: Launch road Fen; verbatimLatitude: 58.754; verbatimLongitude: -93.998; verbatimEventDate: 19-Jul-2010; lifeStage: Adult; catalogNumber: 10PROBE-29276; recordedBy: Brandon Laforest; otherCatalogNumbers: JBHCH968-10; identifiedBy: Jose Fernandez-Triana; institutionID: Biodiversity Institute of Ontario

g. country: Canada; stateProvince: Manitoba; county: Churchill; locality: 23 km E Churchill, Fort Churchill; verbatimElevation: 27 ; verbatimLatitude: 58.734 ; verbatimLongitude: -93.789; verbatimEventDate: 07-Aug-1952; lifeStage: Adult; catalogNumber: HYM00000798; recordedBy: J.G.Chillcot; otherCatalogNumbers: ASWAS997-07; identifiedBy: Jose Fernandez-Triana; institutionID: Canadian National Collection of Insects, Arachnids and Nematodes

h. $\quad$ country: Canada; stateProvince: Newfoundland and Labrador; county: Cap St-George; locality: Cap St-George, fallowfield \& coastal tundra; verbatimLatitude: 48.466; verbatimLongitude: -59.260; verbatimEventDate: 11-Jul-2008; lifeStage: Adult; catalogNumber: CAM0958; recordedBy: H. Goulet, A. Badiss, C. Boudeault;

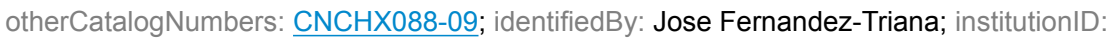
Canadian National Collection of Insects, Arachnids and Nematodes

i. $\quad$ country: Canada; stateProvince: Yukon Territory; locality: Tahkini River road; verbatimElevation: 693; verbatimLatitude: 60.875; verbatimLongitude: -135.386 ; verbatimEventDate: 06-Jul-2006; lifeStage: Adult; catalogNumber: HYM00000981; recordedBy: Goulet \& Boudrealt; otherCatalogNumbers: DSWAS952-07; identifiedBy: Jose Fernandez-Triana; institutionID: Canadian National Collection of Insects, Arachnids and Nematodes

j. $\quad$ country: Canada; stateProvince: Alberta; county: Elk Island NP; municipality: penninsula in Astotin Lake; locality: The Point near administration/warden office; verbatimElevation: 719; verbatimLatitude: 53.685 ; verbatimLongitude: -112.860 ; verbatimEventDate: $10-$ Jun-2012; lifeStage: Adult; catalogNumber: BIOUG03191-A01; recordedBy: Stephanie Church; otherCatalogNumbers: CNEIB1042-12; identifiedBy: Angela Telfer; institutionID: Biodiversity Institute of Ontario

k. country: Canada; stateProvince: Manitoba; county: Churchill; locality: Fort Churchill; verbatimLatitude: 58.734; verbatimLongitude: -93.820; verbatimEventDate: 21-Jul-1952; lifeStage: Adult; catalogNumber: HYM00000792.2; recordedBy: J. G. Chillcot; otherCatalogNumbers: ASWAY590-08; identifiedBy: Jose Fernandez-Triana; institutionID: Canadian National Collection of Insects, Arachnids and Nematodes

I. country: Canada; stateProvince: Ontario; county: Almonte; locality: 5 km NW of Almonte, Hwy 49, Burnt Land, Alvar Prov. Park; verbatimLatitude: 45.255; verbatimLongitude: -76.140; verbatimEventDate: 29-May-2008; lifeStage: Adult; catalogNumber: CAM0427;

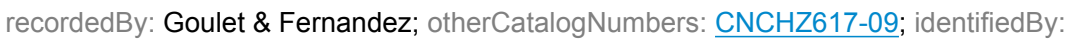
Jose Fernandez-Triana; institutionID: Canadian National Collection of Insects, Arachnids and Nematodes

m. country: Canada; stateProvince: Manitoba; county: Churchill; locality: 23 km E Churchill, Fort Churchill; verbatimElevation: 27; verbatimLatitude: 58.734 ; verbatimLongitude: 
-93.789; verbatimEventDate: 21-Jun-1952; lifeStage: Adult; catalogNumber: HYM00000795; recordedBy: J.G.Chillcot; otherCatalogNumbers: ASWAS994-07; identifiedBy: Jose Fernandez-Triana; institutionID: Canadian National Collection of Insects, Arachnids and Nematodes

n. country: Canada; stateProvince: Manitoba; county: Churchill; locality: $23 \mathrm{~km}$ E Churchill, Fort Churchill; verbatimElevation: 27 ; verbatimLatitude: 58.734 ; verbatimLongitude: -93.789; verbatimEventDate: 07-Nov-1952; lifeStage: Adult; catalogNumber: HYM00000813; recordedBy: J.G.Chillcot; otherCatalogNumbers: DSWAS756-07; identifiedBy: Jose Fernandez-Triana; institutionID: Canadian National Collection of Insects, Arachnids and Nematodes

o. country: Canada; stateProvince: Nova Scotia; locality: Kejimkujik National Park, Peter's Point; verbatimLatitude: 44.366; verbatimLongitude: -65.196; verbatimEventDate: 02Aug-2009; lifeStage: Adult; catalogNumber: GOU 0582; recordedBy: J. Fernandez; otherCatalogNumbers: WOMIA352-11; identifiedBy: Jose Fernandez-Triana; institutionID: Canadian National Collection of Insects, Arachnids and Nematodes

p. $\quad$ country: Canada; stateProvince: Manitoba; county: Churchill; municipality: $23 \mathrm{~km} \mathrm{E}$ Churchill, Ramsay Creek; verbatimElevation: 10; verbatimLatitude: 58.731; verbatimLongitude: -93.780; verbatimEventDate: 23-Jul-2007; lifeStage: Adult; catalogNumber: 07PROBE-23399; recordedBy: A.Renaud; otherCatalogNumbers: ASWAX965-08; identifiedBy: Angela Telfer; institutionID: Biodiversity Institute of Ontario

q. country: Canada; stateProvince: Manitoba; county: Churchill; locality: 23 km E Churchill, Fort Churchill; verbatimElevation: 27 ; verbatimLatitude: 58.734 ; verbatimLongitude: -93.789; verbatimEventDate: 11-Jul-1952; lifeStage: Adult; catalogNumber: HYM00000922; recordedBy: J.G.Chillcot; otherCatalogNumbers: DSWAS865-07; identifiedBy: Jose Fernandez-Triana; institutionID: Canadian National Collection of Insects, Arachnids and Nematodes

r. country: Canada; stateProvince: Ontario; county: Manitoulin Island; locality: Little Current prairie Alvar; verbatimLatitude: 45.975 ; verbatimLongitude: -81.904 ; verbatimEventDate: 04-Jun-2008; lifeStage: Adult; catalogNumber: CAM0412; recordedBy: Goulet, Boudreault \& Fernandez; otherCatalogNumbers: CNCHZ602-09; identifiedBy: Jose Fernandez-Triana; institutionID: Canadian National Collection of Insects, Arachnids and Nematodes

S. $\quad$ country: Canada; stateProvince: Manitoba; county: Churchill; municipality: $23 \mathrm{~km} \mathrm{E}$ Churchill, Ramsay Creek; verbatimElevation: 10; verbatimLatitude: 58.731; verbatimLongitude: -93.780; verbatimEventDate: 23-Jul-2007; lifeStage: Adult; catalogNumber: 07PROBE-23413; recordedBy: A.Renaud; otherCatalogNumbers: ASWAX979-08; identifiedBy: Jose Fernandez-Triana; institutionID: Biodiversity Institute of Ontario

t. country: Canada; stateProvince: British Columbia; county: Yoho NP; municipality: Kicking Horse Cmpgrd.; locality: Amphitheatre flowered meadow; verbatimElevation: 1317; verbatimLatitude: 51.424; verbatimLongitude: -116.429; verbatimEventDate: 21-Jul-2010; lifeStage: Adult; catalogNumber: 10BBCHY-2887; recordedBy: BIObus 2010; otherCatalogNumbers: BBHYK928-10; identifiedBy: Julie K. Stahlhut; institutionID: Biodiversity Institute of Ontario

u. country: Canada; stateProvince: Ontario; county: Almonte; locality: 5 km NW of Almonte, Hwy 49, Burnt Land, Alvar Prov. Park; verbatimLatitude: 45.255; verbatimLongitude: -76.140; verbatimEventDate: 29-May-2008; lifeStage: Adult; catalogNumber: CAM0422; recordedBy: Goulet \& Fernandez; otherCatalogNumbers: CNCHZ612-09; identifiedBy: Jose Fernandez-Triana; institutionID: Canadian National Collection of Insects, Arachnids and Nematodes 
v. country: Canada; stateProvince: Yukon Territory; locality: Pelly Crossing; verbatimElevation: 495; verbatimLatitude: 62.831; verbatimLongitude: -136.585 ; verbatimEventDate: 15-Jul-2006; lifeStage: Adult; catalogNumber: HYM00000945; recordedBy: Goulet \& Boudrealt; otherCatalogNumbers: DSWAS916-07; identifiedBy: Jose Fernandez-Triana; institutionID: Canadian National Collection of Insects, Arachnids and Nematodes

w. country: Canada; stateProvince: Manitoba; county: Churchill; locality: 23 km E Churchill, Fort Churchill; verbatimElevation: 27; verbatimLatitude: 58.734; verbatimLongitude: -93.789; verbatimEventDate: 21-Jul-1952; lifeStage: Adult; catalogNumber: HYM00000814.2; recordedBy: J.G.Chillcot; otherCatalogNumbers: ASWAY595-08; identifiedBy: Jose Fernandez-Triana; institutionID: Canadian National Collection of Insects, Arachnids and Nematodes

x. country: Canada; stateProvince: Ontario; county: Almonte; locality: 5 km NW of Almonte, Hwy 49, Burnt Land, Alvar Prov. Park; verbatimLatitude: 45.255; verbatimLongitude: -76.140; verbatimEventDate: 29-May-2008; lifeStage: Adult; catalogNumber: CAM0435;

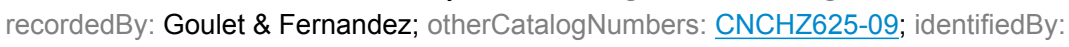
Jose Fernandez-Triana; institutionID: Canadian National Collection of Insects, Arachnids and Nematodes

y. $\quad$ country: Canada; stateProvince: Manitoba; county: Churchill; locality: 23 km E Churchill, Fort Churchill; verbatimElevation: 27; verbatimLatitude: 58.734; verbatimLongitude: -93.789; verbatimEventDate: 07-Aug-1952; lifeStage: Adult; catalogNumber: HYM00000801; recordedBy: J.G.Chillcot; otherCatalogNumbers: DSWAS744-07; identifiedBy: Jose Fernandez-Triana; institutionID: Canadian National Collection of Insects, Arachnids and Nematodes

z. country: Canada; stateProvince: Newfoundland and Labrador; county: Cap St-George; locality: Cap St-George, fallowfield \& coastal tundra; verbatimLatitude: 48.466; verbatimLongitude: -59.260; verbatimEventDate: 11-Jul-2008; lifeStage: Adult; catalogNumber: CAM0959; recordedBy: H. Goulet, A. Badiss, C. Boudeault; otherCatalogNumbers: CNCHX089-09; identifiedBy: Jose Fernandez-Triana; institutionID: Canadian National Collection of Insects, Arachnids and Nematodes

aa. country: Canada; stateProvince: Yukon Territory; locality: Tahkini River road; verbatimElevation: 693; verbatimLatitude: 60.875; verbatimLongitude: -135.386; verbatimEventDate: 06-Jul-2006; lifeStage: Adult; catalogNumber: HYM00000964; recordedBy: Goulet \& Boudrealt; otherCatalogNumbers: DSWAS935-07; identifiedBy: Jose Fernandez-Triana; institutionID: Canadian National Collection of Insects, Arachnids and Nematodes

ab. country: Canada; stateProvince: Manitoba; county: Churchill; locality: 23 km E Churchill, Fort Churchill; verbatimElevation: 27; verbatimLatitude: 58.734; verbatimLongitude: -93.789; verbatimEventDate: 16-Jul-1952; lifeStage: Adult; catalogNumber: HYM00000794; recordedBy: J.G.Chillcot; otherCatalogNumbers: ASWAS993-07; identifiedBy: Jose Fernandez-Triana; institutionID: Canadian National Collection of Insects, Arachnids and Nematodes

ac. country: Canada; stateProvince: Manitoba; county: Churchill; municipality: 23 km E Churchill, Ramsay Creek; verbatimElevation: 10; verbatimLatitude: 58.731; verbatimLongitude: -93.780; verbatimEventDate: 16-Jul-2007; lifeStage: Adult; catalogNumber: 07PROBE-22417; recordedBy: A.Renaud; otherCatalogNumbers: ASWAV958-08; identifiedBy: Angela Telfer; institutionID: Biodiversity Institute of Ontario ad. country: Canada; stateProvince: Nova Scotia; locality: Kejimkujik National Park, Peter`s Point; verbatimLatitude: 44.366; verbatimLongitude: -65.196; verbatimEventDate: 02Aug-2009; lifeStage: Adult; catalogNumber: GOU 0590; recordedBy: J. Fernandez; 
otherCatalogNumbers: WOMIA360-11; identifiedBy: Jose Fernandez-Triana; institutionID: Canadian National Collection of Insects, Arachnids and Nematodes

ae. $\quad$ country: Canada; stateProvince: Newfoundland and Labrador; county: Plum Point; locality: Plum Point, fallow field; verbatimLatitude: 51.064; verbatimLongitude: -56.982; verbatimEventDate: 16-Jul-2008; lifeStage: Adult; catalogNumber: CAM0936;

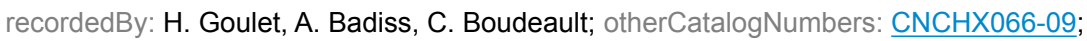
identifiedBy: Jose Fernandez-Triana; institutionID: Canadian National Collection of Insects, Arachnids and Nematodes

af. country: Canada; stateProvince: Manitoba; county: Churchill; municipality: $23 \mathrm{~km} \mathrm{E}$ Churchill, Ramsay Creek; verbatimElevation: 10; verbatimLatitude: 58.731; verbatimLongitude: -93.780; verbatimEventDate: 21-Jul-2007; lifeStage: Adult; catalogNumber: 07PROBE-20806; recordedBy: A.Renaud; otherCatalogNumbers: ASWAT346-08; identifiedBy: Jose Fernandez-Triana; institutionID: Biodiversity Institute of Ontario

ag. country: Canada; stateProvince: Ontario; county: Almonte; locality: 5 km NW of Almonte, Hwy 49, Burnt Land, Alvar Prov. Park; verbatimLatitude: 45.255; verbatimLongitude: -76.140; verbatimEventDate: 29-May-2008; lifeStage: Adult; catalogNumber: CAM0434; recordedBy: Goulet \& Fernandez; otherCatalogNumbers: CNCHZ624-09; identifiedBy: Jose Fernandez-Triana; institutionID: Canadian National Collection of Insects, Arachnids and Nematodes

ah. country: Canada; stateProvince: Newfoundland and Labrador; county: Sally`s Cove; locality: Sally`s Cove, fallow field; verbatimLatitude: 49.739; verbatimLongitude: -57.925 ; verbatimEventDate: 13-Jul-2008; lifeStage: Adult; catalogNumber: CAM0948;

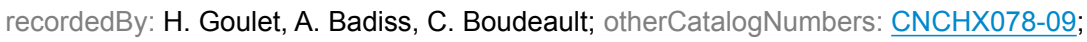
identifiedBy: Jose Fernandez-Triana; institutionID: Canadian National Collection of Insects, Arachnids and Nematodes

ai. country: Canada; stateProvince: Manitoba; county: Churchill; locality: 23 km E Churchill, Fort Churchill; verbatimElevation: 27 ; verbatimLatitude: 58.734 ; verbatimLongitude: -93.789; verbatimEventDate: 04-Aug-1952; lifeStage: Adult; catalogNumber: HYM00000802; recordedBy: J.G.Chillcot; otherCatalogNumbers: DSWAS745-07; identifiedBy: Jose Fernandez-Triana; institutionID: Canadian National Collection of Insects, Arachnids and Nematodes

aj. $\quad$ country: Canada; stateProvince: Yukon Territory; locality: Fish Lake Rd; verbatimElevation: 1107; verbatimLatitude: 60.686; verbatimLongitude: -135.236; verbatimEventDate: 05-Jul-2006; lifeStage: Adult; catalogNumber: HYM00001682; recordedBy: Goulet \& Boudreault; otherCatalogNumbers: ASWAU310-08; identifiedBy: Jose Fernandez-Triana; institutionID: Canadian National Collection of Insects, Arachnids and Nematodes

ak. country: Canada; stateProvince: Ontario; county: Almonte; locality: $5 \mathrm{~km} \mathrm{NW}$ of Almonte, Hwy 49, Burnt Land, Alvar Prov. Park; verbatimLatitude: 45.255; verbatimLongitude: -76.140; verbatimEventDate: 29-May-2008; lifeStage: Adult; catalogNumber: CAM0421; recordedBy: Goulet \& Fernandez; otherCatalogNumbers: CNCHZ611-09; identifiedBy: Jose Fernandez-Triana; institutionID: Canadian National Collection of Insects, Arachnids and Nematodes

al. country: Canada; stateProvince: Ontario; county: Almonte; locality: 5 km NW of Almonte, Hwy 49, Burnt Land, Alvar Prov. Park; verbatimLatitude: 45.255; verbatimLongitude: -76.140; verbatimEventDate: 29-May-2008; lifeStage: Adult; catalogNumber: CAM0416; recordedBy: Goulet \& Fernandez; otherCatalogNumbers: CNCHZ606-09; identifiedBy: Jose Fernandez-Triana; institutionID: Canadian National Collection of Insects, Arachnids and Nematodes 
am. country: Canada; stateProvince: Manitoba; county: Churchill; locality: 23 km E Churchill, Fort Churchill; verbatimElevation: 27 ; verbatimLatitude: 58.734 ; verbatimLongitude: -93.789; verbatimEventDate: 01-Jan-1952; lifeStage: Adult; catalogNumber: HYM00000815; recordedBy: J.G.Chillcot; otherCatalogNumbers: DSWAS758-07; identifiedBy: Jose Fernandez-Triana; institutionID: Canadian National Collection of Insects, Arachnids and Nematodes

an. country: Canada; stateProvince: Ontario; county: Almonte; locality: 5 km NW of Almonte, Hwy 49, Burnt Land, Alvar Prov. Park; verbatimLatitude: 45.255; verbatimLongitude: -76.140; verbatimEventDate: 29-May-2008; lifeStage: Adult; catalogNumber: CAM0428; recordedBy: Goulet \& Fernandez; otherCatalogNumbers: CNCHZ618-09; identifiedBy: Jose Fernandez-Triana; institutionID: Canadian National Collection of Insects, Arachnids and Nematodes

ao. country: Canada; stateProvince: Manitoba; county: Churchill; locality: Warkworth Creek nr. Churchill; verbatimLatitude: 58.700; verbatimLongitude: -94.100 ; verbatimEventDate: 08-May-1952; lifeStage: Adult; catalogNumber: HYM00000816; recordedBy: J.G.Chillcot; otherCatalogNumbers: DSWAS759-07; identifiedBy: Jose Fernandez-Triana; institutionID: Canadian National Collection of Insects, Arachnids and Nematodes

ap. country: Canada; stateProvince: Manitoba; county: Churchill; municipality: $23 \mathrm{~km} \mathrm{E}$ Churchill, Ramsay Creek; verbatimElevation: 10; verbatimLatitude: 58.731; verbatimLongitude: -93.780; verbatimEventDate: 21-Jul-2007; lifeStage: Adult; catalogNumber: 07PROBE-20818; recordedBy: A.Renaud; otherCatalogNumbers: ASWAT358-08; identifiedBy: Jose Fernandez-Triana; institutionID: Biodiversity Institute of Ontario

aq. country: Canada; stateProvince: Ontario; county: Manitoulin Island; locality: Little Current prairie Alvar; verbatimLatitude: 45.975; verbatimLongitude: -81.904; verbatimEventDate: 04-Jun-2008; lifeStage: Adult; catalogNumber: CAM0386; recordedBy: Goulet, Boudreault \& Fernandez; otherCatalogNumbers: CNCHZ576-09; identifiedBy: Jose Fernandez-Triana; institutionID: Canadian National Collection of Insects, Arachnids and Nematodes

ar. country: Canada; stateProvince: Ontario; county: Manitoulin Island; locality: Little Current prairie Alvar; verbatimLatitude: 45.975; verbatimLongitude: -81.904; verbatimEventDate: 04-Jun-2008; lifeStage: Adult; catalog Number: CAM0387; recordedBy: Goulet, Boudreault \& Fernandez; otherCatalogNumbers: CNCHZ577-09; identifiedBy: Jose Fernandez-Triana; institutionID: Canadian National Collection of Insects, Arachnids and Nematodes

as. Country: Canada; stateProvince: Newfoundland and Labrador; county: Sally`s Cove; locality: Sally`s Cove, fallow field; verbatimLatitude: 49.739; verbatimLongitude: -57.925 ; verbatimEventDate: 13-Jul-2008; lifeStage: Adult; catalogNumber: CAM0944; recordedBy: H. Goulet, A. Badiss, C. Boudeault; otherCatalogNumbers: CNCHX074-09; identifiedBy: Jose Fernandez-Triana; institutionID: Canadian National Collection of Insects, Arachnids and Nematodes

at. country: United States; stateProvince: Colorado; locality: Boulder; verbatimElevation: 1828.82; verbatimEventDate: 31-May-1961; lifeStage: Adult; catalogNumber: CNCHYM 03216; recordedBy: B. H. Poole; otherCatalogNumbers: HYCNF001-11; identifiedBy: J. Whitfield; institutionID: Canadian National Collection of Insects, Arachnids and Nematodes

au. country: Canada; stateProvince: Newfoundland and Labrador; county: South Branch; locality: South Branch, fallow field; verbatimLatitude: 47.922; verbatimLongitude: -59.014; verbatimEventDate: 10-Jul-2008; lifeStage: Adult; catalogNumber: CAM0952; recordedBy: H. Goulet, A. Badiss, C. Boudeault; otherCatalogNumbers: CNCHX082-09; 
identifiedBy: Jose Fernandez-Triana; institutionID: Canadian National Collection of Insects, Arachnids and Nematodes

av. country: Canada; stateProvince: Manitoba; county: Churchill; locality: 23 km E Churchill, Fort Churchill; verbatimElevation: 27 ; verbatimLatitude: 58.734 ; verbatimLongitude: -93.789; verbatimEventDate: 01-Jul-1952; lifeStage: Adult; catalogNumber: HYM00000815.2; recordedBy: J.G.Chillcot; otherCatalogNumbers: ASWAY594-08; identifiedBy: Jose Fernandez-Triana; institutionID: Canadian National Collection of Insects, Arachnids and Nematodes

aw. country: Canada; stateProvince: Ontario; county: Almonte; locality: 5 km NW of Almonte, Hwy 49, Burnt Land, Alvar Prov. Park; verbatimLatitude: 45.255; verbatimLongitude: -76.140; verbatimEventDate: 29-May-2008; lifeStage: Adult; catalogNumber: CAM0426; recordedBy: Goulet \& Fernandez; otherCatalogNumbers: CNCHZ616-09; identifiedBy: Jose Fernandez-Triana; institutionID: Canadian National Collection of Insects, Arachnids and Nematodes

ax. country: Canada; stateProvince: Manitoba; county: Churchill; municipality: $11 \mathrm{~km} \mathrm{~S}$ Churchill, Goose Creek; locality: Goose Creek Second Train Tracks; verbatimLatitude: 58.718; verbatimLongitude: -94.122; verbatimEventDate: 16-Jul-2010; lifeStage: Adult; catalogNumber: 10PROBE-28643; recordedBy: Brandon Laforest; otherCatalogNumbers: JBHCH335-10; identifiedBy: Jose Fernandez-Triana; institutionID: Biodiversity Institute of Ontario

ay. country: Canada; stateProvince: Manitoba; county: Churchill; locality: $23 \mathrm{~km}$ E Churchill, Fort Churchill; verbatimElevation: 27 ; verbatimLatitude: 58.734 ; verbatimLongitude: -93.789; verbatimEventDate: 11-Jul-1952; lifeStage: Adult; catalogNumber: HYM00000811.2; recordedBy: J.G.Chillcot; otherCatalogNumbers: ASWAY598-08; identifiedBy: Jose Fernandez-Triana; institutionID: Canadian National Collection of Insects, Arachnids and Nematodes

az. country: Canada; stateProvince: Newfoundland and Labrador; locality: Sally`s Cove, fallow field; verbatimLatitude: 49.739 ; verbatimLongitude: -57.925 ; verbatimEventDate: 13-Jul-2008; lifeStage: Adult; catalogNumber: CAM0492; recordedBy: H. Goulet, A. Badiss, C. Boudeault; otherCatalogNumbers: $\mathrm{CNCHZ1139-09;} \mathrm{identifiedBy:} \mathrm{Jose}$ Fernandez-Triana; institutionID: Canadian National Collection of Insects, Arachnids and Nematodes

ba. $\quad$ country: Canada; stateProvince: Newfoundland and Labrador; county: Sally`s Cove; locality: Sally`s Cove, fallow field; verbatimLatitude: 49.739; verbatimLongitude: -57.925 ; verbatimEventDate: 13-Jul-2008; lifeStage: Adult; catalogNumber: CAM0945; recordedBy: H. Goulet, A. Badiss, C. Boudeault; otherCatalogNumbers: CNCHX075-09; identifiedBy: Jose Fernandez-Triana; institutionID: Canadian National Collection of Insects, Arachnids and Nematodes

bb. $\quad$ country: Canada; stateProvince: Yukon Territory; locality: Fish Lake road; verbatimElevation: 1107; verbatimLatitude: 60.688; verbatimLongitude: -135.238 ; verbatimEventDate: 05-Jul-2006; lifeStage: Adult; catalogNumber: HYM00000603; recordedBy: Goulet \& Boudrealt; otherCatalogNumbers: $\mathrm{CNCHY331-07;} \mathrm{identifiedBy:}$ Jose Fernandez-Triana; institutionID: Canadian National Collection of Insects, Arachnids and Nematodes

bc. country: Canada; stateProvince: Newfoundland and Labrador; county: Plum Point; locality: Plum Point, fallow field; verbatimLatitude: 51.064; verbatimLongitude: -56.982; verbatimEventDate: 16-Jul-2008; lifeStage: Adult; catalogNumber: CAM0951; recordedBy: H. Goulet, A. Badiss, C. Boudeault; otherCatalogNumbers: CNCHX081-09; identifiedBy: Jose Fernandez-Triana; institutionID: Canadian National Collection of Insects, Arachnids and Nematodes 
bd. $\quad$ country: Canada; stateProvince: Yukon Territory; locality: Fish Lake road; verbatimElevation: 1107; verbatimLatitude: 60.688; verbatimLongitude: -135.238; verbatimEventDate: 05-Jul-2006; lifeStage: Adult; catalogNumber: HYM00000989; recordedBy: Goulet \& Boudrealt; otherCatalogNumbers: DSWAS960-07; identifiedBy: Jose Fernandez-Triana; institutionID: Canadian National Collection of Insects, Arachnids and Nematodes

be. country: Canada; stateProvince: Newfoundland and Labrador; locality: Sally`s Cove, fallow field; verbatimLatitude: 49.739 ; verbatimLongitude: -57.925 ; verbatimEventDate: 13-Jul-2008; lifeStage: Adult; catalogNumber: MIC 000900; recordedBy: H. Goulet, A. Badiss, C. Boudeault; otherCatalogNumbers: $\mathrm{CNCHZ1092-09;} \mathrm{identifiedBy:} \mathrm{Jose}$ Fernandez-Triana; institutionID: Canadian National Collection of Insects, Arachnids and Nematodes

bf. $\quad$ country: Canada; stateProvince: Manitoba; county: Churchill; municipality: $23 \mathrm{~km} \mathrm{E}$ Churchill, Ramsay Creek; verbatimElevation: 10; verbatimLatitude: 58.731; verbatimLongitude: -93.780; verbatimEventDate: 21-Jul-2007; lifeStage: Adult; catalogNumber: 07PROBE-20808; recordedBy: A.Renaud; otherCatalogNumbers: ASWAT348-08; identifiedBy: Jose Fernandez-Triana; institutionID: Biodiversity Institute of Ontario

bg. country: Canada; stateProvince: Ontario; county: Almonte; locality: 5 km NW of Almonte, Hwy 49, Burnt Land, Alvar Prov. Park; verbatimLatitude: 45.255; verbatimLongitude: -76.140; verbatimEventDate: 29-May-2008; lifeStage: Adult; catalogNumber: CAM0365; recordedBy: Goulet \& Fernandez; otherCatalogNumbers: CNCHZ555-09; identifiedBy: Jose Fernandez-Triana; institutionID: Canadian National Collection of Insects, Arachnids and Nematodes

bh. country: Canada; stateProvince: Ontario; county: Manitoulin Island; locality: Little Current, prairie alvar; verbatimLatitude: 45.975; verbatimLongitude: -81.904; verbatimEventDate: 04-Jun-2008; lifeStage: Adult; catalogNumber: CAM0384; recordedBy: Goulet,

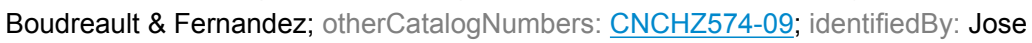
Fernandez-Triana; institutionID: Canadian National Collection of Insects, Arachnids and Nematodes

bi. country: Canada; stateProvince: Ontario; county: Almonte; locality: 5 km NW of Almonte, Hwy 49, Burnt Land, Alvar Prov. Park; verbatimLatitude: 45.255; verbatimLongitude: -76.140; verbatimEventDate: 29-May-2008; lifeStage: Adult; catalogNumber: CAM0431; recordedBy: Goulet \& Fernandez; otherCatalogNumbers: CNCHZ621-09; identifiedBy: Jose Fernandez-Triana; institutionID: Canadian National Collection of Insects, Arachnids and Nematodes

Notes: This species is widely distributed in the Holarctic (e.g., Whitfield 2006, Fernandez-Triana 2010, Yu et al. 2012, Fernandez-Triana et al. 2014). Here we present new and detailed information about the distribution of the species in Canada, including one new provincial record $(\mathrm{ON})$, based on examined specimens from the BIO and CNC collections. Data for those specimens was extracted from BOLD.

\section{Discussion}

In addition to being a diverse platform for the generation, analysis and permanent storage of DNA barcodes, the Barcode of Life Data Systems (BOLD) houses an immense amount of collateral data that are only starting to be explored and exploited. Validated species 
occurrences records are one such data element, critical for various fields and applications from environmental niche modeling in ecology to risk assessments in conservation biology. This paper demonstrates the potential of using BOLD occurrence data for these purposes - to define and refine the distributions of species - and presents a model for their rapid dissemination in the primary literature.

The key to this model is the seamless and dynamic transition of data between source and journal. By converting specimen information (such as locality, collection date, collector, voucher depository) from the BOLD platform to the Excel template provided by the Pensoft Writing Tool (PWT), the authoring platform of the Biodiversity Data Journal (BDJ), it is possible to quickly upload and generate long lists of "Material Examined" for papers discussing taxonomy, ecology and/or new distribution records of species. Additional functions in BOLD allow verification of this material, for instance the ability to generate high-res image libraries (e.g., Suppl. material 3) or Kimura 2-Parameter (K2P) phylograms using the corresponding sequences (e.g., Suppl. material 4). For the vast majority of publications including DNA barcodes, the generation and publication of ancillary data associated with the barcoded material is seldom highlighted and often disregarded. The analysis of those data sets to uncover new distribution patterns of species has rarely been explored, even though many BOLD records represent new and/or significant discoveries (but see Smith et al. (2012) for a discussion of this topic). The introduction of journals specializing in - and streamlining - the release of these datasets, such as the BDJ, should facilitate thorough analysis of these records, even large datasets. This paper used a moderately large data set (630 specimens comprising 10 species) and revealed several new distribution records, as well as the workflow that permitted their release (i.e., from a database to a publication).

The logical next step would be to create a simple interface and module at the source database (BOLD) that will submit data selected by the author, "at the click of a button", into the PWT, using the Application Programming Interface (API) of the latter. Within the PWT environment, the submitting author can complete the manuscript by adding additional text, figures, images, citations, references and so on. The author can also invite co-authors and/ or "contributors" (e.g., linguistic editors, mentors, colleagues who are not formal coauthors) to collaboratively work on the manuscript. After completion, the manuscript is submitted to BDJ, again "at the click of a button", where it undergoes a community peerreview process and subsequent publication upon acceptance (e.g., Smith et al. 2013). The published article will contain dynamic links to external sources (Penev et al. 2010), including to BOLD, and will also be available in highly structured XML format which ensures machine readability of the text and data. For example, the occurrence records will automatically be exported to GBIF and appear there with their original source (e.g., BOLD) and publication source (reference for citation), bringing additional visibility and usage tracking statistics to both the source database and the authors. Visibility and usability are essential here, particularly for collateral data such as species occurrence records residing on BOLD, as the consumers and eventual uses of this biodiversity data are nearly as diverse as the organisms they represent. 


\section{Acknowledgements}

The work of Pensoft on this project was party supported by the EC-FP7 EU BON project (grant agreement №308454). Barcode analysis was facilitated by funding from the government of Canada through Genome Canada and the Ontario Genomics Institute in support of the International Barcode of Life project. The development of the Barcode of Life Data Systems (BOLD) was enabled by funding from the Ontario Ministry of Research and Innovation. JFT is grateful for the technical support of Teodor Georgiev and Pavel Stoev (Pensoft).

\section{References}

- $\quad$ de Huiza R (1995) Diversidad de Braconidae (Hymenoptera) en el Peru. Revista Peruana de Entomología 37: 11-22.

- Fernandez-Triana J (2010) Eight new species and an annotated checklist of Microgastrinae (Hymenoptera, Braconidae) from Canada and Alaska. ZooKeys 63: 1-53. DOI: $10.3897 /$ zookeys.63.565

- Fernandez-Triana J, Shaw M, Cardinal S, Mason P (2014) Contributions to the study of the Holarctic fauna of Microgastrinae (Hymenoptera, Braconidae). I. Introduction and first results of transatlantic comparisons. Journal of Hymenoptera Research 37: 61-76. DOI: $10.3897 / j \mathrm{jhr} .37 .7186$

- $\quad$ Fernández-Triana J, Huber J (2010) Braconid parasitoids (Hymenoptera: Braconidae) of Nearctic Choristoneura species (Lepidoptera: Tortricidae), with a summary of other parasitoid families attacking Choristoneura. The Canadian Entomologist 142: 295-343. DOI: $\underline{10.4039 / n 10-025}$

- Hellén W (1977) För Finlands fauna nya Apanteles-arter (Hymenoptera, Braconidae). Notulae Entomologicae 57: 9-10.

- Penev L, Agosti D, Georgiev T, Catapano T, Miller J, Blagoderov V, Roberts D, Smith V, Brake I, Ryrcroft S, Scott B, Johnson N, Morris R, Sautter G, Chavan V, Robertson T, Remsen D, Stoev P, Parr C, Knapp S, Kress W, Thompson C, Erwin T (2010) Semantic tagging of and semantic enhancements to systematics papers: ZooKeys working examples. ZooKeys 50: 1-16. DOI: 10.3897/zookeys.50.538

- Ratnasingham S, Hebert PD (2013) A DNA-Based Registry for All Animal Species: The Barcode Index Number (BIN) System. PLoS ONE 8 (7): e66213. DOI: 10.1371/ journal.pone.0066213

- $\quad$ Smith MA, Fernández-Triana JL, Eveleigh E, Gómez J, Guclu C, Hallwachs W, Hebert PDN, Hrcek J, Huber JT, Janzen D, Mason PG, Miller S, Quicke DLJ, Rodriguez JJ, Rougerie R, Shaw MR, Várkonyi G, Ward DF, Whitfield JB, Zaldívar-Riverón A (2012) DNA barcoding and the taxonomy of Microgastrinae wasps (Hymenoptera, Braconidae): impacts after 8 years and nearly 20000 sequences. Molecular Ecology Resources 13 (2): 168-176. DOI: 10.1111/1755-0998.12038

- Smith V, Georgiev T, Stoev P, Biserkov J, Miller J, Livermore L, Baker E, Mietchen D, Couvreur TLP, Mueller G, Dikow T, Helgen KM, Frank J, Agosti D, Roberts D, Penev L (2013) Beyond dead trees: integrating the scientific process in the Biodiversity Data Journal. Biodiversity data journal 1: e995. DOI: 10.3897/BDJ.1.e995 
- Whitfield JB (1997) Subfamily Microgastrinae. In: Wharton RA, Marsh PM, Sharkey MJ (Eds) Manual of the New World genera of the family Braconidae. International Society of Hymenopterists, Washington, D.C 1: 333-364.

- Whitfield JB (2006) Revision of the Nearctic species of the genus Pholetesor Mason (Hymenoptera: Braconidae). Zootaxa 1144: 1-94.

- Yu DS, van Achterberg C, Horstmann K (2012) Taxapad 2012, Ichneumonoidea 2011. Database on flash-drive. URL: www.taxapad.com

\section{Supplementary materials}

\section{Suppl. material 1: Supplemental Appendix 1}

Authors: Fernandez-Triana et al. 2014

Data type: Complete Specimen Data downloaded from BOLD

Filename: Supplemental Appendix 1.xls - Download file $(783.50 \mathrm{~kb})$

\section{Suppl. material 2: Supplemental Appendix 2}

Authors: Fernandez-Triana et al. 2014

Data type: Data from BOLD modified for BDJ template

Filename: Supplemental Appendix 2.xls - Download file (315.00 kb)

\section{Suppl. material 3: Supplemental Appendix 3}

Authors: Fernández-Triana et al. 2014

Data type: Image Library of specimens studied

Filename: Image Library in BOLD of specimens studied.pdf - Download file (904.10 kb)

\section{Suppl. material 4: Supplemental Appendix 4}

Authors: Fernández-Triana et al. 2014

Data type: K2P tree of sequences studied

Filename: K2P tree of specimens studied.pdf - Download file $(22.67 \mathrm{~kb})$

\section{Suppl. material 5: Distribution of 10 Microgastrinae species based on BOLD records}

Authors: Fernández-Triana et al. 2014

Data type: Distribution records

Filename: Maps.pdf - Download file (5.51 MB) 